\title{
An inverse micro-mechanical analysis toward the stochastic homogenization of nonlinear random composites
}

\author{
Ling $\mathrm{Wu}^{\mathrm{a}, *}$, Van-Dung Nguyen ${ }^{\mathrm{a}}$, Laurent Adam ${ }^{\mathrm{b}}$, Ludovic Noels ${ }^{\mathrm{a}}$ \\ ${ }^{a}$ University of Liege, Department of Mechanical and Aerospace Engineering, \\ Computational \&3 Multiscale Mechanics of Materials, Allée de la découverte 9, B-4000 \\ Liège, Belgium \\ ${ }^{b}$-Xstream Engineering, Axis Park-Building H, Rue Emile Francqui 9, B-1435 \\ Mont-Saint-Guibert, Belgium
}

\begin{abstract}
An inverse Mean-Field Homogenization (MFH) process is developed to improve the computational efficiency of non-linear stochastic multiscale analyzes by relying on a micro-mechanics model. First full-field simulations of composite Stochastic Volume Element (SVE) realizations are performed to characterize the homogenized stochastic behavior. The uncertainties observed in the non-linear homogenized response, which result from the uncertainties of their micro-structures, are then translated to an incrementalsecant MFH formulation by defining the MFH input parameters as random effective properties. These effective input parameters, which correspond to the micro-structure geometrical information and to the material phases model parameters, are identified by conducting an inverse analysis from the full-field homogenized responses. Compared to the direct finite element analyzes on SVEs, the resulting stochastic MFH process reduces not only the computational cost, but also the order of uncertain parameters in the composite micro-structures, leading to a stochastic Mean-Field Reduced Order Model (MF-ROM). A data-driven stochastic model is then built in order to generate the random effective properties under the form of a random field used
\end{abstract}

\footnotetext{
${ }^{*}$ Corresponding author, Phone: +32 436694 53, Fax: +32 43669505

Email addresses: L.Wu@ulg.ac. be (Ling Wu ), VanDung.Nguyen@ulg.ac.be (Van-Dung Nguyen), laurent.adam@e-xstream.com (Laurent Adam), L. Noels@ulg.ac.be (Ludovic Noels)
} 
as entry for the stochastic MF-ROM embedded in a Stochastic Finite Element Method (SFEM). The two cases of elastic Unidirectional (UD) fibers embedded in an elasto-plastic matrix and of elastic UD fibers embedded in a damage-enhanced elasto-plastic matrix are successively considered. In order to illustrate the capabilities of the method, the stochastic response of a ply is studied under transverse loading condition.

Keywords: Stochastic, Homogenization, Micro-Mechanics, Composite Materials, Data-driven

\section{Introduction}

In the last decade there has been a growing interest in considering uncertainties in structural engineering. One source of uncertainties originates from the material itself, and is of particular importance when considering strongly non-linear behaviors and/or fracture $[1,2]$. This is the case in composite materials for which variability in the micro-structure leads to a variability in the structural quantity of interest such as the strength. Although Stochastic Finite Elements (SFEM) [3, 4] provide a mean of assessing the structural stochastic response, they require the definition of proper random fields [5], which can hardly be fully characterized from experimental data, since a limited number of mechanical tests cannot accurately sample the distribution of a material response. This has led to the conclusion that the random fields should be defined from micro-mechanical information $[5,6,7]$, paving the way to virtual testing.

In the context of virtual testing of composite materials, the uncertainties in the micro-structure arrangement are experimentally characterized and statistically represented in order to be able to generate synthetic microstructures having the same stochastic features and that can be readily used in numerical simulations. We refer to the works $[8,9,10]$ for woven composites, [11] for particle-reinforced composites and [12, 13, 14] for unidirectional fiber reinforced composites (UD). While for the former case, the generated structures can be envisioned to be used for structural studies [8, 9], for the latter two heterogeneous materials, because the length scales are separated by several orders of magnitudes [5], a homogenization step is first required.

Different multiscale techniques, see the reviews [15, 16, 17], have been developed analytically and/or numerically to predict the macro or mesoscopic response of heterogeneous materials at reduced computational costs 


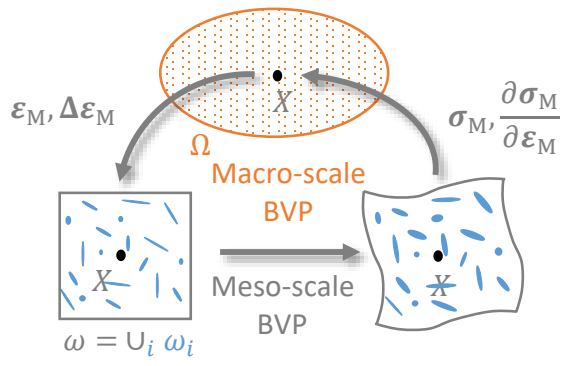

(a)

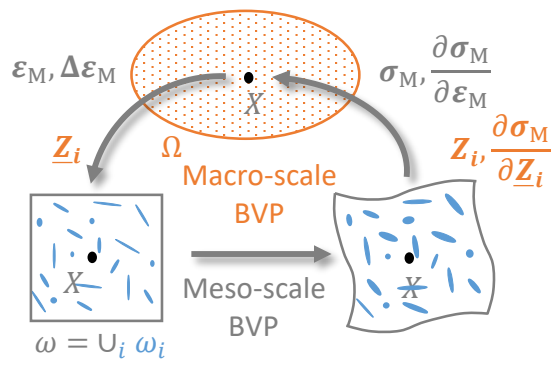

(b)

Figure 1: Multiscale analysis performed by homogenization of the meso-scale volume element $\omega$ : (a) Non-Linear multiscale analysis; (b) Multiscale analysis using a non-local counterpart $\underline{\mathbf{Z}}_{i}$ of a homogenized representation of an internal variable $\mathbf{Z}_{i}$ in phase $\omega_{i}$. The latter are not rigorously volume averaged values.

while maintaining a high degree of accuracy. Among them, computational homogenization based strategies were developed in [18, 19, 20, 21, 22] and have gained popularity with the increase in computational power. In a homogenization-based multiscale analysis, at each (macro) material point of interest, a Boundary Value Problem (BVP) is defined on a meso-scale volume, which represents the different phases of the material, see Fig. 1(a). Computational homogenization investigates the response of the meso-scale volume element through a full-field analysis like the Finite Element (FE) method, in which case the multiscale method is also called Finite Element Square $\left(\mathrm{FE}^{2}\right)$. Since the micro-structures and phases properties of heterogeneous materials are considered explicitly in the meso-scale volume elements, the computational homogenization can provide rather accurate predictions.

Computational homogenization-based multiscale methods have mainly been developed by considering a deterministic micro-scale BVP by assuming statistical representativity of the latter, allowing the definition of a Representative Volume Element (RVE) on which the homogenization is achieved. However, a RVE cannot always be defined: because of the various sources of uncertainties, which are involved in real micro-structures, the size of the RVE may reach the order of the macro-scale for some heterogeneous materials, such as UD fiber and/or woven fiber reinforced matrix material. This increase in the RVE size is particularly severe for non-linear behaviors such as plasticity [23, 2] or micro-cracking [2]. In that case, a unique RVE cannot be used since the scale separation criterion for a multiscale analysis is not 
satisfied. In order to be able to predict by virtual testing the uncertainties observed at the structural scale, the multiscale analyzes should thus be conducted on the material synthetic micro-structures which capture the material stochasticity; these synthetic micro-structures serve as Stochastic or Statistical Volume Elements (SVEs) [7] in the context of homogenization: local homogenized properties depend on the SVE realization, and on the applied boundary conditions as studied in the case of elasticity $[14,23,24]$, in the case in micropolar elasticity [25, 26], in the non-linear case [27, 23], and in limit analyzes [28].

Although, the distribution of the homogenized properties of SVE realizations can be used to define apparent homogenized properties [23, 24, 25, 26], in the context of virtual testing and stochastic analyzes, the SVEs are seen as a mean to propagate the micro-scale uncertainties to a higher scale. However, direct $\mathrm{FE}^{2}$ methods conducted on SVEs and combined to macro-scale SFEM are not computationally affordable, in particular when Monte Carlo (MC) simulations are conducted at the structural scale. Indeed, in order to conduct a reliable stochastic multiscale structural analysis by SFEM, the stochastic description of the material properties should be defined accounting for the size of the stochastic finite elements [29]: the SVEs used to define the meso-scale random material property fields should have a comparable size to the finite element size in the structural analysis, putting severe constraints on the finite element mesh used at the SFEM level. Therefore, in the context of linear elastic materials, to avoid the costly coupled micro-scale computations, phenomenological macroscopic models were stochastically calibrated from homogenized responses of computational homogenization performed on SVEs: a transverse isotropic law with resultant Young's modulus and Poisson ratio was calibrated for the homogenized behavior of random two-phase composites [30,31] and an orthogonal anisotropic law was adopted for the same material system [14], for poly-silicon elasticity [29], and for poly-silicon thermo-elastic damping [32].

Nevertheless, in the context of non-linear materials it is not always easy to define an ad hoc model since the behavior of the materials system can be complex. Using a modified version of the meso-scale stochastic finite element method (SFEM), the stochastic homogenization of a UD composite cell can be achieved [33] efficiently, but the micro-structure is limited to a single fiber. An alternative is to speed up the homogenization process by considering a Reduced Order Model (ROM). In general, a ROM accelerates the micro-scale analyzes with pre-off-line computations. Based on the acquired information 
from pre-off-line full-field micro-scale analyzes, the full-order governing equations are projected into a suitably selected reduced order space. In order to avoid the use of a large number of displacement degrees of freedom, the microscale model is solved with a reduced number of unknown variables which are defined by means of proper orthogonal decomposition of the displacement field $[34,35]$. Furthermore, hyper-reduction techniques can be applied to reduce the computation cost of the internal forces resulting from the evaluation of the local constitutive equations $[36,37]$. In the context of order reduction using a micro-mechanics-based homogenization model, nonuniform transformation field analysis (NTFA) [38] reduces the field of internal variables by using the pre-defined internal variables modes obtained with pre-off-line fullfield analyzes. Moreover, a tangent second-order (TSO) expansion of the dissipation potential [39] corresponds to a reduction of the evolution equations related to the reduced internal variables. An alternative to the TSOexpansion is the NTFA extension in terms of a potential-based Reduced Basis Model Order Reduction (pRBMOR), developed in [40], which provides the evolution law for the reduced degrees of freedom from a mixed incremental variational principle, and which has been combined to GPU acceleration in [41]. By dividing the volume element phases into clusters of similar strains obtained through full-field analyzes and by performing a Hashin-Shtrikman homogenization, an efficient order reduction method for non-linear reversible behaviors has been developed in [42]. Clustering the volume elements from linear analyzes and applying a self consistent homogenization of the nonlinear clusters response is an efficient alternative proposed in [43]. Finally, the mapping functions, as surrogate models, can also be constructed from pre-off-line computations, such as through kernel methods [44], polynomial chaos expansion [45] etc.

The concept of ROMs has been used in stochastic analyzes. Using an order reduction method combined to an asymptotic homogenization on SVEs, stochastic multiscale analyzes account for fine-scale material properties as random variables -and random fields in particular cases [46]. By defining a meso-scale potential which reflects the uncertainties related to the fibers geometry/distribution in composites, a ROM was built from the resolution of SVEs in the context of finite elasticity $[47,48]$. The idea of using data-driven ROMs in non-linear stochastic analyzes is also discussed in [49]. Nevertheless ROMs remain mainly applied to deterministic analyzes, in which the pre-offline full-field analyzes need to be carried out on a RVE, which is by definition statistically representative. Indeed, using ROM to solve MC resolutions of 
realistic SVEs would not only require a huge series of pre-off-line computations on SVEs, but the memory required to store these information from off-line computations also becomes overwhelming. Addressing the problem of conducting efficient stochastic multiscale analyzes in the context of virtual testing remains thus highly challenging.
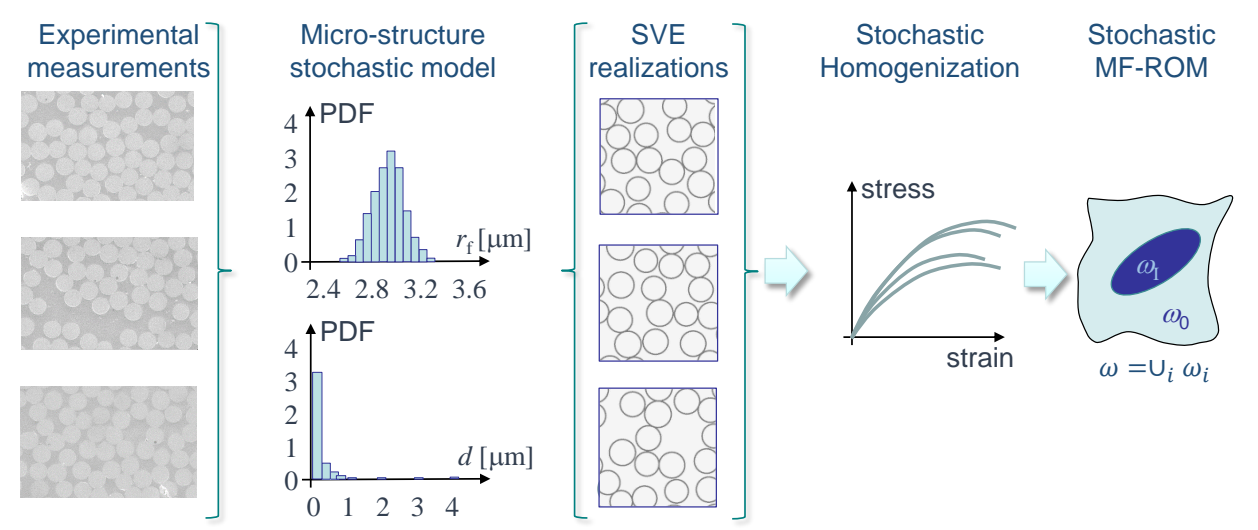

Figure 2: Development of a stochastic non-linear Mean-Field Reduced Order Model (MFROM).

The purpose of this paper is to define a stochastic non-linear micromechanics model serving as meso-scale surrogate model, which represents the non-deterministic material response of UD composite materials, and that can be used as random field input of a SFEM analysis. The developed methodology is described in Fig. 2. Using SEM images of composite materials, a micro-structure stochastic model was developed in [14] from statistical representation, such as the fiber radius $r_{\mathrm{f}}$ and nearest distance $d$ illustrated in Fig. 2, allowing synthetic micro-structures to be generated under the form of SVE realizations of arbitrary size and number. Conducting non-linear full-field analyzes and performing homogenization on these SVE realizations lead to a set of homogenized responses, which are in turn used to identify the random effective material properties of the micro-mechanics-based surrogate model. In this context, the non-linear micro-mechanics model is seen as a ROM basis in which the order reduction actually corresponds to the identification of the effective material parameters.

In [50], such a methodology has been developed in the context of linear elasticity by using as stochastic ROM basis the non-linear Mean-Field Homogenization (MFH) theory, thus defining a stochastic Mean-Field Reduced 
Order Model (MF-ROM). The MFH approach is originally a computationally efficient semi-analytical framework for the modeling of multi-phase composites. MFH methods were first developed for linear elastic composites using different extensions of the Eshelby single inclusion solution [51] to account for the multiple inclusions interaction, as in the Mori-Tanaka [52, 53] and in the self-consistent $[54,55]$ schemes. Based on the concept of Linear Comparison Composite (LCC) [56, 57], MFH has been extended to the modeling of non-linear composites whose constituents may exhibit non-linear behaviors, as plasticity, visco-elasticity ... By opposition to computational homogenization, the microstructure is not explicitly represented but defined from the phases material properties, the phases volume fractions and either from the inclusion geometrical shape or from their spatial correlation [58]; the BVP is then formulated by a series of equations, completed by the knowledge of phases properties, that can be solved iteratively. Since the detailed spacial information of the micro-structure is neglected, the volume element is only a concept in the MFH and the micro-structure uncertainty effects cannot be directly accounted for in contrast to the computational homogenization method. Therefore, in this work, the MFH theory is not used as a predictive homogenization method, but as a ROM basis in which the uncertainties are represented by a few random parameters defined as random effective material properties and geometrical parameters identified through an inverse micro-mechanical analysis conducted on the SVE full-field resolutions.

In this paper this MF-ROM concept is developed for non-linear elastoplastic and damage-enhanced elasto-plastic responses of UD composite materials. The main interest of considering the stochastic MF-ROM is its computational efficiency allowing stochastic scale-transition to be carried out by the SFEM for non-linear behaviors and macro-scale quantity of interest, such as the strength, to be assessed as it will be illustrated on a virtual ply loading test.

The paper is organized as follows. General background for homogenizationbased multiscale analyzes is given in Section 2 for both the $\mathrm{FE}^{2}$ and the $\mathrm{MFH}$ methods that will be used in this work for the full-field analysis and the definition of the MF-ROM, respectively. The stochastic MF-ROM is defined in Section 3 by conducting an inverse identification of the model parameters from the full-field analyzes. First the case of linear elasticity treated in [50] is briefly summarized, then the cases of elastic fibers embedded in elasto-plastic and damage-enhanced elasto-plastic matrix are successively developed. Note that in the case of damage-enhanced elasto-plasticity, the formalism pre- 
sented is strictly valid up to the strain-softening onset of the meso-scale homogenized behavior. During strain softening, the homogenized behavior of the SVEs obtained by computational homogenization in terms of a bulk stress-strain relationship is not valid since size objectivity is not ensured [59]. The developed methodology is then applied in the context of UD composites in Section 4, and the stochastic responses predicted by the MF-ROM are compared to the full-field simulation results. The statistical characteristics of the realizations of the MF-ROM effective parameters identified for the UD composites are then studied in Section 5. From these realizations, a stochastic model is built in order to generate random vectors of the effective properties respecting these information. Since there exists a dependence between the properties, and since only a limited number of samples is available, we adopt the data-driven sampling method developed in [60]. As a result, random vectors can be generated and used as entry for the stochastic MFROM embedded in a Stochastic Finite Element Method (SFEM). Finally, the applicability of the method to study a stochastic response at a higher scale is investigated in Section 6. It is shown that, although in the linear range the ply response remains deterministic, the non-linear response exhibits uncertainties as well as the ply strength. However, since we do not account for the loss of size objectivity during softening of the homogenized response, the stochastic study of the ply strength is only presented to illustrate the potential of the method while future developements are still required.

\section{Homogenization-based multiscale analysis}

In this section, the main equations of the homogenization-based multiscale analysis are reviewed. In the multiscale approach, at each macro-point $\boldsymbol{X}$, the macro-strain tensor $\varepsilon_{\mathrm{M}}$ and its increment $\Delta \varepsilon_{\mathrm{M}}$ are known, and the macro-stress tensor $\boldsymbol{\sigma}_{\mathrm{M}}$ is sought from the resolution of a meso-scale Boundary Value Problem (BVP), see Fig. 1(a). To this end, the macro-point is viewed as the center of a meso-scale volume element of domain $\boldsymbol{x} \in \omega$. First the governing equations at the micro-scale are given in the cases of anisotropic elasticity and $J_{2}$-elasto-plastic phase materials. Elasto-plasticity is successively considered with and without damage. Then, two homogenization methods for non-linear composites are reviewed: the computational homogenization or $\mathrm{FE}^{2}$ method and the incremental-secant $\mathrm{MFH}$. 


\subsection{Definition of the meso-scale boundary value problem}

At the micro-scale, any point $\boldsymbol{x} \in \omega$ belongs to a phase $\omega_{i}$ with $\cup_{i} \omega_{i}=\omega$. At that scale the classical continuum mechanics is assumed to hold. Besides we assume that the deformations remain infinitesimal and that the time for a stress wave to propagate in the meso-scale volume element remains negligible. Therefore, in the absence of dynamics effects the equilibrium equations read

$$
\left\{\begin{array}{l}
\nabla_{\mathrm{m}} \cdot \boldsymbol{\sigma}_{\mathrm{m}}=0 \quad \forall \boldsymbol{x} \in \omega, \\
\boldsymbol{n}_{\mathrm{m}} \cdot \boldsymbol{\sigma}_{\mathrm{m}}=\boldsymbol{t}_{\mathrm{m}} \quad \forall \boldsymbol{x} \in \partial \omega,
\end{array}\right.
$$

where the subscript 'm' refers to the local value at the micro-scale, $\boldsymbol{\sigma}_{\mathrm{m}}$ is the Cauchy stress tensor, and $\boldsymbol{t}_{\mathrm{m}}$ is the surface traction on the boundary $\partial \omega$ of outward unit normal $\boldsymbol{n}_{\mathrm{m}}$. Notations are detailed in Appendix A.

To complete the micro-scale problem, the local constitutive laws of the different materials are written as

$$
\boldsymbol{\sigma}_{\mathrm{m}}(t)=\boldsymbol{\sigma}_{\mathrm{m}}\left(\varepsilon_{\mathrm{m}}(t) ; \mathbf{Z}_{\mathrm{m}}(\tau), \tau \in[0, t]\right),
$$

where the small-deformation strain tensor $\varepsilon_{\mathrm{m}}$ is evaluated in terms of the micro-scale displacement $\boldsymbol{u}_{\mathrm{m}}$ as $\boldsymbol{\varepsilon}_{\mathrm{m}}=\frac{1}{2}\left(\nabla_{\mathrm{m}} \otimes \boldsymbol{u}_{\mathrm{m}}+\boldsymbol{u}_{\mathrm{m}} \otimes \nabla_{\mathrm{m}}\right)$, and where $\mathbf{Z}_{\mathrm{m}}$ is a set of internal variables allowing history-dependent processes to be accounted for.

Futhermore, anticipating on the case in which a damaging process is considered, a subset of the internal variables $\mathbf{Z}_{\mathrm{m}}(\tau)$ ought to be considered in a non-local form in order to avoid mesh dependency during the strain softening stage (locally in the meso-scale volume element $\omega$ ). The non-local internal variables denoted as $\underline{\mathbf{Z}}_{\mathrm{m}}$ are herein evaluated using the implicit nonlocal model $[61,62,63,64]$, in which the relation between an internal variable $p \in \mathbf{Z}_{\mathrm{m}}$ to its non-local counterpart $\underline{p} \in \underline{\mathbf{Z}}_{\mathrm{m}}$ follows the Helmholtz-type equation

$$
\underline{p}-\nabla \cdot \boldsymbol{c}_{\mathrm{g}} \cdot \nabla \underline{p}=p \quad \forall \boldsymbol{x} \in \omega,
$$

where $\boldsymbol{c}_{\mathrm{g}}$ is the matrix of the squared characteristic lengths [65] introduced to account for material anisotropy. In the case of an isotropic medium $\boldsymbol{c}_{\mathrm{g}}=$ $\operatorname{diag}\left(l^{2} ; l^{2} ; l^{2}\right)$ with the characteristic length scale $l$. Under this non-local framework, the constitutive Eq. (2) becomes

$$
\boldsymbol{\sigma}_{\mathrm{m}}(t)=\boldsymbol{\sigma}_{\mathrm{m}}\left(\varepsilon_{\mathrm{m}}(t), \underline{\mathbf{Z}}_{\mathrm{m}}(\tau) ; \mathbf{Z}_{\mathrm{m}}(\tau), \tau \in[0, t]\right) .
$$

We can now define the constitutive equations that will be considered in this work. 


\subsubsection{Model of elastic (possibly anisotropic) phases}

For elastic phases, the constitutive Eq. (2) is rewritten in the linear form

$$
\boldsymbol{\sigma}_{\mathrm{m}}=\mathbb{C}_{\mathrm{m}}^{\mathrm{el}}: \varepsilon_{\mathrm{m}}
$$

where the fourth-order material tensor $\mathbb{C}_{\mathrm{m}}^{\mathrm{el}}(\boldsymbol{x})$ can be anisotropic.

In the transverse isotropic case, the material parameters are the transverse Young's modulus $E^{\mathrm{T}}$, the transverse Poisson ratio $\nu^{\mathrm{TT}}$, the longitudinaltransverse Poisson ratio $\nu^{\mathrm{LT}}$, the transverse shear modulus $\mu^{\mathrm{TT}}$, the longitudinaltransverse shear modulus $\mu^{\mathrm{LT}}$, and the longitudinal Young's modulus $E^{\mathrm{L}}$.

\subsubsection{Material models of (possibly damage-enhanced) $J_{2}$-elasto-plastic phases}

The constitutive Eq. (4) is now detailed in the case of elasto-plastic materials enhanced by a non-local damage model. For the case of materials without damage, these equations still hold by enforcing explicitly the damage parameter to zero (i.e. $D_{\mathrm{m}}=0$ ).

Considering the strain equivalence assumption for which the strain tensors observed in the actual body and in its undamaged representation are equivalent [66], the average undamaged stress ${ }^{1}$ is defined by introducing a damage parameter and reads

$$
\hat{\boldsymbol{\sigma}}_{\mathrm{m}}=\frac{\boldsymbol{\sigma}_{\mathrm{m}}}{\left(1-D_{\mathrm{m}}\right)}
$$

where $\boldsymbol{\sigma}_{\mathrm{m}}$ is the apparent stress and where the damage variable satisfies $0 \leq D_{\mathrm{m}}<1$. The damage evolution is formulated in a non-local constitutive equation

$$
\left\{\begin{array}{l}
\dot{D}_{\mathrm{m}}(t)=\mathcal{D}\left(D_{\mathrm{m}}(t), \varepsilon_{\mathrm{m}}(t), \chi(t) ; \mathbf{Z}_{\mathrm{m}}\left(t^{\prime}\right), t^{\prime} \in[0, t]\right) \dot{\chi}, \\
\chi(t)=\max _{t^{\prime} \in[0, t]}\left(p_{C}, \underline{p}\left(t^{\prime}\right)\right)
\end{array}\right.
$$

where $\chi$ is the maximum of the damage threshold $p_{C}$ and of the maximum non-local internal variable $p$ reached during the process. This non-local variable is related to the local internal variable $p$ through Eq. (3). For conciseness, the subscript " $\mathrm{m}$ " is dropped in the following equations.

\footnotetext{
${ }^{1}$ In the literature this quantity is sometimes called effective stress; however since we use the term "effective" for identified properties, and in order to avoid confusion, we called it undamaged stress in this paper.
} 
Assuming an elasto-plastic material, which obeys $J_{2}$ elasto-plasticity, the von Mises stress criterion reads

$$
f=\hat{\sigma}^{\mathrm{eq}}-R(p)-\sigma_{Y} \leqslant 0 .
$$

In this expression, $f$ is the yield surface, $\hat{\sigma}^{\text {eq }}=\sqrt{\frac{3}{2} \frac{\operatorname{dev}(\boldsymbol{\sigma})}{1-D}: \frac{\operatorname{dev}(\boldsymbol{\sigma})}{1-D}}$ is the equivalent von Mises undamaged stress, $\sigma_{Y}$ is the initial yield stress, $R(p) \geqslant 0$ is the isotropic hardening stress, and $p$ is the internal variable characterizing the irreversible behavior ${ }^{2}$. During the occurrence of plastic flow, $f=0, \dot{p}$ is positive, and the plastic strain tensor increment obeys the normal plastic flow, which is summarized by

$$
\dot{\boldsymbol{\varepsilon}}^{\mathrm{pl}}=\dot{p} \boldsymbol{N}, \quad \text { with } \boldsymbol{N}=\frac{\partial f}{\partial \hat{\boldsymbol{\sigma}}}=\frac{3}{2} \frac{\operatorname{dev}(\boldsymbol{\sigma})}{(1-D) \hat{\sigma}^{\mathrm{eq}}},
$$

where $\boldsymbol{N}$ is the normal to the yield surface in the undamaged stress space.

In this formalism, the internal variable $p$ thus represents the accumulated plastic strain $\dot{p}=\left[\frac{2}{3} \dot{\varepsilon}^{\mathrm{pl}}: \dot{\varepsilon}^{\mathrm{pl}}\right]^{1 / 2}$, and, assuming small deformations, the reversible (elastic) and irreversible (plastic) strain tensors can be added $(\varepsilon=$ $\left.\varepsilon^{\mathrm{el}}+\varepsilon^{\mathrm{pl}}\right)$, yielding

$$
\boldsymbol{\sigma}=(1-D) \mathbb{C}^{\mathrm{el}}:\left(\varepsilon-\varepsilon^{\mathrm{pl}}\right)
$$

where $\mathbb{C}^{\mathrm{el}}$ is the fourth-order Hooke tensor of the undamaged material. The radial return mapping equations of this damage formalism and their linearization are summarized in Appendix B.

\subsection{Scale transition}

The scale transition defines the relation between macro-strains $\varepsilon_{\mathrm{M}}$ and stresses $\boldsymbol{\sigma}_{\mathrm{M}}$ into the relation between average micro-strains $\left\langle\boldsymbol{\varepsilon}_{\mathrm{m}}(\boldsymbol{x})\right\rangle_{\omega}$ and stresses $\left\langle\boldsymbol{\sigma}_{\mathrm{m}}(\boldsymbol{x})\right\rangle_{\omega}$ over the meso-scale volume element $\omega$, with

$$
\varepsilon_{\mathrm{M}}=\left\langle\varepsilon_{\mathrm{m}}(\boldsymbol{x})\right\rangle_{\omega} \quad \text { and } \quad \boldsymbol{\sigma}_{\mathrm{M}}=\left\langle\boldsymbol{\sigma}_{\mathrm{m}}(\boldsymbol{x})\right\rangle_{\omega}
$$

\footnotetext{
${ }^{2}$ Rigorously, the von Mises stress criterion (8) should be written $f(\hat{\boldsymbol{\sigma}}, r) \leqslant 0$, where $r$ is an internal variable related to the accumulated plastic strain $p$ and to the plastic multiplier $\dot{\lambda}$ following $\dot{r}=\dot{\lambda}=(1-D) \dot{p}$, see the discussion by [67] for details. However in this paper we use the approximation that consists in writing the J2-plasticity in the undamaged stress space.
} 
where $\langle f(\boldsymbol{x})\rangle_{\omega}=\frac{1}{V_{\omega}} \int_{\omega} f(\boldsymbol{x}) \mathrm{d} V$ and $V_{\omega}$ is the volume of the meso-scale volume element $\omega$.

We now define two homogenization methods that can be used to perform the scale transition: the computational homogenization or $\mathrm{FE}^{2}$ method and the MFH method.

\subsubsection{Computational homogenization}

The problem of computational homogenization is illustrated in Fig. 1(a), in which an equivalent macro-scale strain-stress response is extracted from the resolution of the meso-scale BVP discretized using the finite-element method.

Hill-Mandel condition. The energy consistency between the different scales, which corresponds to the Hill-Mandel condition, is stated as

$$
\boldsymbol{\sigma}_{\mathrm{M}}: \delta \varepsilon_{\mathrm{M}}=\left\langle\boldsymbol{\sigma}_{\mathrm{m}}: \delta \varepsilon_{\mathrm{m}}\right\rangle_{\omega}
$$

The micro-scale displacement field is written under the form

$$
\boldsymbol{u}_{\mathrm{m}}(\boldsymbol{x})=\varepsilon_{\mathrm{M}} \cdot\left(\boldsymbol{x}-\boldsymbol{x}_{\mathrm{ref}}\right)+\boldsymbol{u}^{\prime}(\boldsymbol{x}),
$$

where $\boldsymbol{x}_{\text {ref }}$ is a reference point of $\omega$ and $\boldsymbol{u}^{\prime}$ is the perturbation field. Considering the definition of the homogenized strain tensor $\varepsilon_{\mathrm{M}}$, Eq. (11), this perturbation field should thus satisfy the condition

$$
0=\left\langle\nabla_{\mathrm{m}} \otimes \boldsymbol{u}^{\prime}(\boldsymbol{x})+\boldsymbol{u}^{\prime}(\boldsymbol{x}) \otimes \nabla_{\mathrm{m}}\right\rangle_{\omega}=\frac{1}{V_{\omega}} \int_{\partial \omega}\left(\boldsymbol{n}_{\mathrm{m}} \otimes \boldsymbol{u}^{\prime}+\boldsymbol{u}^{\prime} \otimes \boldsymbol{n}_{\mathrm{m}}\right) \mathrm{d} S .
$$

Besides, substituting Eq. (13) in Eq. (12), integrating by parts, and using the equilibrium Eqs. (1), allow the Hill-Mandel condition (12) to be rewritten as

$$
\boldsymbol{\sigma}_{\mathrm{M}}: \delta \varepsilon_{\mathrm{M}}=\left\langle\boldsymbol{\sigma}_{\mathrm{m}}: \delta \varepsilon_{\mathrm{m}}\right\rangle_{\omega}=\boldsymbol{\sigma}_{\mathrm{M}}: \delta \varepsilon_{\mathrm{M}}+\frac{1}{V_{\omega}} \int_{\omega} \boldsymbol{\sigma}_{\mathrm{m}}:\left(\delta \boldsymbol{u}^{\prime} \otimes \nabla_{\mathrm{m}}\right) \mathrm{d} V,
$$

or again as

$$
0=\int_{\partial \omega}\left(\boldsymbol{\sigma}_{\mathrm{m}} \cdot \boldsymbol{n}_{\mathrm{m}}\right) \cdot \delta \boldsymbol{u}^{\prime} \mathrm{d} S=\int_{\partial \omega} \boldsymbol{t}_{\mathrm{m}} \cdot \delta \boldsymbol{u}^{\prime} \mathrm{d} S
$$


Definition of the constrained micro-scale finite element problem. Let $\delta \boldsymbol{u}^{\prime} \in \mathcal{U}$ be a test function, where $\mathcal{U}$ is an admissible kinematic vector field subset of the minimum kinematic field $\mathcal{U}^{\text {min }}$ satisfying Eq. (14), i.e.

$$
\mathcal{U}^{\min }=\left\{\delta \boldsymbol{u}^{\prime} \mid \int_{\partial \omega}\left(\boldsymbol{n}_{\mathrm{m}} \otimes \delta \boldsymbol{u}^{\prime}+\delta \boldsymbol{u}^{\prime} \otimes \boldsymbol{n}_{\mathrm{m}}\right) \mathrm{d} S=0\right\} .
$$

Then, the weak form of the micro-scale Eqs. (1) reads

$$
\int_{\omega} \boldsymbol{\sigma}_{\mathrm{m}}:\left(\delta \boldsymbol{u}^{\prime} \otimes \nabla_{\mathrm{m}}\right) \mathrm{d} V=0, \quad \forall \delta \boldsymbol{u}^{\prime} \in \mathcal{U} .
$$

Since $\mathcal{U} \subset \mathcal{U}^{\min }$, the resolution of this weak form always ensures Eq. (15) and the Hill-Mandel condition (12) is satisfied.

The variational statement (18) of the Hill-Mandel condition was introduced in $[68,69]$ and is practically implemented by defining specific boundary conditions on the meso-scale volume element whose constraint is to satisfy Eq. (14), as detailed in [70].

Following the study on the effect of boundary conditions conducted in [14] for linear responses, we consider the Periodic Boundary Conditions (PBCs), for which the admissible kinematic vector field $\mathcal{U}$ is defined by

$$
\begin{aligned}
\mathcal{U}^{\mathrm{PBC}}=\left\{\boldsymbol{u}^{\prime} \mid \boldsymbol{u}_{\mathrm{m}}\left(\boldsymbol{x}^{+}\right)-\boldsymbol{u}_{\mathrm{m}}\left(\boldsymbol{x}^{-}\right)=\boldsymbol{\varepsilon}_{\mathrm{M}} \cdot\left(\boldsymbol{x}^{+}-\boldsymbol{x}^{-}\right),\right. \\
\left.\forall \boldsymbol{x}^{+} \in \partial \omega^{+} \text {and corresponding } \boldsymbol{x}^{-} \in \partial \omega^{-}\right\} \subset \mathcal{U}^{\mathrm{min}},
\end{aligned}
$$

where the parallelepiped SVE faces have been separated in opposite surfaces $\partial \omega^{-}$and $\partial \omega^{+}$. Note that the variational statement does not require the PBCs to constrain directly the symmetry of the surface traction in order to satisfy the Hill-Mandel condition. This symmetry is a consequence of the micro-scale problem resolution as shown by considering arbitrary $\delta \boldsymbol{u}^{\prime} \in \mathcal{U}^{\mathrm{PBC}}$ in Eq. (16).

In the case of damage enhanced material model, there is no scale transition considered for the local and non-local internal variables $p$ and $\underline{p}$. The weak form associated to Eq. (3) reads

$$
\int_{\omega}\left\{\left(\underline{p}_{\mathrm{m}}-p_{\mathrm{m}}\right) \delta \underline{p}+\nabla \underline{p}_{\mathrm{m}} \cdot \boldsymbol{c}_{\mathrm{g}} \cdot \nabla \delta \underline{p}\right\} \mathrm{d} V=0, \quad \forall \delta \underline{p} \in \mathcal{K},
$$

where the test function $\delta p$ belong in an admissible kinematic field $\mathcal{K}$. This admissible kinematic field $\mathcal{K}$ depends on the boundary conditions selected for the non-local equations. Following the natural boundary conditions

$$
\boldsymbol{n}_{\mathrm{m}} \cdot \boldsymbol{c}_{\mathrm{g}} \cdot \nabla \underline{p}=0 \quad \forall \boldsymbol{x} \in \partial \omega,
$$


one has $\mathcal{K}=H^{1}(\omega)$. However, when considering the failure process, periodic boundary conditions can advantageously be applied, see the complete discussion in [71].

The set of Eqs. $(18,20)$ is completed by the PBCs (19) and the system is solved using the multiplier elimination method [70]. The fourth order macro-scale material tensor $\mathbb{C}_{\mathrm{M}}=\frac{\partial \sigma_{\mathrm{M}}}{\partial \varepsilon_{\mathrm{M}}}$ can be extracted from this resolution. We note that the fourth order macro-scale material tensor $\mathbb{C}_{\mathrm{M}}$ generally does not correspond to the volume average of the micro-scale material tensor $\mathbb{C}_{\mathrm{m}}$. Finally, in order to perform the MT inverse identification in the case of damage, we also need to extract the material tensor $\mathbb{C}_{M}^{\mathrm{el}} \mathrm{D}$, which corresponds to the homogenized material tensor during an elastic unloading. This extraction follows the same expression as for the material tensor but by considering $\mathbb{C}_{\mathrm{m}}=\left(1-D_{\mathrm{m}}\right) \mathbb{C}_{\mathrm{m}}^{\mathrm{el}}$ in the micro-constituents.

\subsubsection{Incremental-secant Mean-Field Homogenization (MFH)}

The principle of MFH resolution is illustrated in Fig. 1(a). Considering a two-phase isothermal composite material, with the respective volume fractions $v_{0}+v_{\mathrm{I}}=1$ (subscript 0 refers to the matrix and I to the inclusions), Eq. (11) can be rewritten by separating the volume averages on the matrix subdomain $\omega_{0}$ and on the inclusions subdomain $\omega_{\mathrm{I}}$ as

$$
\varepsilon_{\mathrm{M}}=v_{0}\left\langle\varepsilon_{\mathrm{m}}\right\rangle_{\omega_{0}}+v_{\mathrm{I}}\left\langle\varepsilon_{\mathrm{m}}\right\rangle_{\omega_{\mathrm{I}}} \quad \text { and } \quad \boldsymbol{\sigma}_{\mathrm{M}}=v_{0}\left\langle\boldsymbol{\sigma}_{\mathrm{m}}\right\rangle_{\omega_{0}}+v_{\mathrm{I}}\left\langle\boldsymbol{\sigma}_{\mathrm{m}}\right\rangle_{\omega_{\mathrm{I}}}
$$

In what follows, the notations $\left\langle\bullet_{\mathrm{m}}\right\rangle_{\omega_{i}}$ are replaced by $\langle\bullet\rangle_{i}$ for conciseness.

Only the strain tensors (total and incremental) and the stress tensors evaluated for a phase $\omega_{i}$ correspond to the volume average of the field within the phase. Internal variables, damage parameters, elastic and plastic parts of strain tensors, or again material operators related to the homogenized phase $\omega_{i}$ are denoted by $\bullet_{i}$ and are the homogenized representations of the corresponding field in the phase but not necessarily the volume averaged values: in general $\bullet_{i} \neq\langle\bullet\rangle_{i}$.

In particular, the case of non-local damage is illustrated in Fig. 1(b), where the local variables $\boldsymbol{Z}_{i}$ correspond to homogenized representations of the composite phases, but are not necessarily the volume average on the phases. The non-local variables $\underline{\boldsymbol{Z}}_{i}$ are solved at the macro-scale using the diffusion Eq. (3) and also correspond to homogenized representations of the composite material phases and not necessarily to the volume average. 
MFH for linear behaviors. The relation between $\varepsilon_{\mathrm{M}}$ and $\boldsymbol{\sigma}_{\mathrm{M}}$ can be evaluated by completing the Eqs. (22), on the one hand, by the constitutive laws of each phase, e.g. by considering uniform constitutive material tensors $\mathbb{C}_{0}^{\mathrm{el}}$ for the matrix phase and $\mathbb{C}_{\mathrm{I}}^{\mathrm{el}}$ for the inclusions phase,

$$
\langle\boldsymbol{\sigma}\rangle_{0}=\mathbb{C}_{0}^{\mathrm{el}}:\langle\boldsymbol{\varepsilon}\rangle_{0} \quad \text { and } \quad\langle\boldsymbol{\sigma}\rangle_{\mathrm{I}}=\mathbb{C}_{\mathrm{I}}^{\mathrm{el}}:\langle\boldsymbol{\varepsilon}\rangle_{\mathrm{I}},
$$

and, on the other hand, by a relation linking the strain averages per phase, which reads

$$
\langle\varepsilon\rangle_{\mathrm{I}}=\mathbb{B}^{\epsilon}\left(\mathrm{I}, \mathbb{C}_{0}^{\mathrm{el}}, \mathbb{C}_{\mathrm{I}}^{\mathrm{el}}\right):\langle\varepsilon\rangle_{0},
$$

where $\mathbb{B}^{\epsilon}$ is the strain concentration tensor whose expression depends on the assumptions made on the micro-mechanics, and (I) refers to the geometrical information of the inclusions required to evaluate $\mathbb{B}^{\epsilon}$. In this work, the MoriTanaka (M-T) model [52] is considered since it provides good predictions for two-phase composite materials for which the matrix can be clearly identified [72]. In this case, the strain concentration tensor reads

$$
\mathbb{B}^{\epsilon}\left(\mathrm{I}, \mathbb{C}_{0}, \mathbb{C}_{\mathrm{I}}\right)=\left\{\mathbb{I}+\mathbb{S}\left(\mathrm{I}, \mathbb{C}_{0}\right):\left[\left(\mathbb{C}_{0}\right)^{-1}: \mathbb{C}_{\mathrm{I}}-\mathbb{I}\right]\right\}^{-1},
$$

where $\mathbb{C}_{0}$ and $\mathbb{C}_{\mathrm{I}}$ are the considered phase linear operators $\left(\mathbb{C}_{0}^{\mathrm{el}}\right.$ and $\mathbb{C}_{\mathrm{I}}^{\mathrm{el}}$ for linear materials) and where the Eshelby tensor $\mathbb{S}\left(\mathrm{I}, \mathbb{C}_{0}\right)[51]$ depends on the geometry of the inclusion (I) and on the operator $\mathbb{C}_{0}$ of the matrix.

The set of Eqs. (22-24) is rewritten in a general constitutive expression for linear elastic composites as

$$
\boldsymbol{\sigma}_{\mathrm{M}}=\mathbb{C}_{\mathrm{M}}^{\mathrm{el}}\left(\mathrm{I}, \mathbb{C}_{0}^{\mathrm{el}}, \mathbb{C}_{\mathrm{I}}^{\mathrm{el}}, v_{\mathrm{I}}\right): \boldsymbol{\varepsilon}_{\mathrm{M}}
$$

Extension to non-linear behaviors. In a non-linear framework, MFH is carried out in an incremental form through the definition of a so-called Linear Comparison Composite (LCC) [57, 73, 74, 75, 76, 77, 78]. The LCC plays the role of a virtual heterogeneous material, whose constituents linear behaviors, defined through virtual elastic operators, match the linearized behaviors of the real composite material constituents for a given strain state.

Therefore, the MFH equations of a linear composite material can be applied by considering the virtual elastic operators $\mathbb{C}_{0}^{\mathrm{LCC}}$ of the matrix phase and $\mathbb{C}_{\mathrm{I}}^{\mathrm{LCC}}$ of the inclusions phase instead of the elastic operators. The relation between the average incremental strains in the two phases (24) is thus rewritten

$$
\langle\Delta \varepsilon\rangle_{\mathrm{I}}=\mathbb{B}^{\epsilon}\left(\mathrm{I}, \mathbb{C}_{0}^{\mathrm{LCC}}, \mathbb{C}_{\mathrm{I}}^{\mathrm{LCC}}\right):\langle\Delta \varepsilon\rangle_{0},
$$


and equation (22) is rewritten

$$
\Delta \varepsilon_{\mathrm{M}}=v_{0}\langle\Delta \boldsymbol{\varepsilon}\rangle_{0}+v_{\mathrm{I}}\langle\Delta \boldsymbol{\varepsilon}\rangle_{\mathrm{I}} \quad \text { and } \quad \boldsymbol{\sigma}_{\mathrm{M}}=v_{0}\langle\boldsymbol{\sigma}\rangle_{0}+v_{\mathrm{I}}\langle\boldsymbol{\sigma}\rangle_{\mathrm{I}}
$$

Among the possible different linearization techniques developed in order to define the LCC we consider the incremental-secant formulation $[79,80]$. Within this framework, the homogenized relation $\Delta \sigma_{\mathrm{M}}-\Delta \varepsilon_{\mathrm{M}}$ is evaluated from the phases responses $\langle\Delta \boldsymbol{\sigma}\rangle_{i}-\langle\Delta \varepsilon\rangle_{i}$ constructed as being uniform, and does not involve neither the definition nor the use of macro-scale elastic and plastic strain tensors, although they could be estimated from the homogenized response. As a result, in the context of incremental MFH, equivalence between the dissipated energies at both scales is not explicitly enforced. However, when considering a variational MFH formulation [81] an incremental free-energy is defined between the deformation configurations, and on which averaging relations are recovered between the scales.

Incremental-secant $M F H$. The incremental-secant formulation provides a way to define the LCC and the operators $\mathbb{C}_{i}^{\mathrm{LCC}}$ following the methodology illustrated in Fig. 3 and Fig. 4 in the cases of elasto-plastic and damage-enhanced elasto-plastic materials, respectively.

Considering a time step $\left[t_{n}, t_{n+1}\right]$, in the incremental-secant formulation the composite material is first subjected to a fictitious elastic unloading from its configuration at time $t_{n}$, see Fig. 3(a) and Fig. 4(a). Note that this unloading remains virtual since a real one could imply reverse plasticity; its purpose is to obtain a virtually unloaded state from which the LCC is defined. This fictitious unloading of the composite material corresponds to the existence of residual stress in its phases as illustrated in Fig. 3(b) and Fig. 4(b). The composite material is then loaded to the new configuration at time $t_{n+1}$, see Fig. 3(c) and Fig. 4(c). The LCC is then defined by considering the secant operators in the phases defined from the residual strain and stress, see Fig. 3(d) and Fig. 4(d).

This definition of the LCC has several advantages as compared to other formulations. First, the isotropization step required by the affine and incremental-tangent methods [82] is avoided because the secant operators are naturally isotropic. Second, the method has a better accuracy in the case of non-proportional loading [79] and in the case of damage-enhanced elastoplasticity since the virtual unloading step allows capturing a phase elastic unloading during softening of another one [80]. 


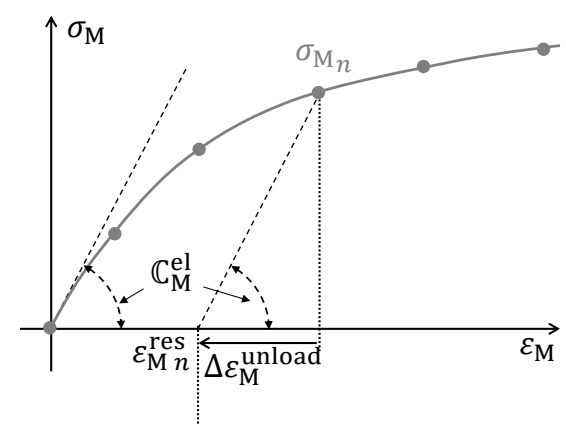

(a) Composite; unloading

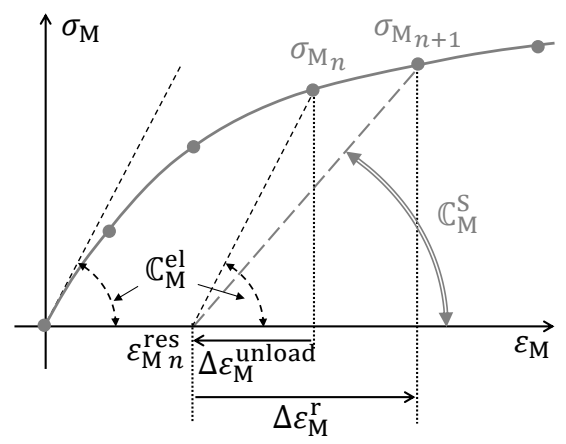

(c) Composite; loading

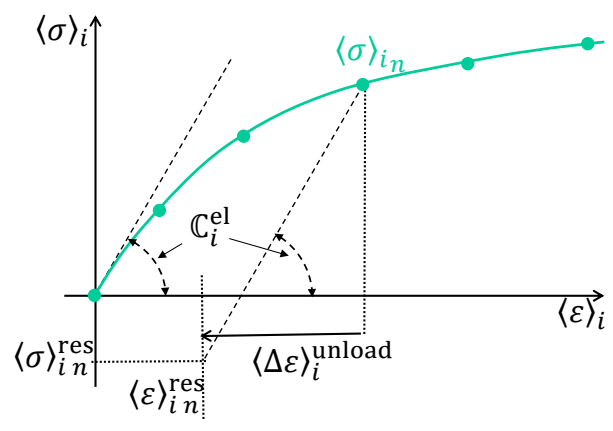

(b) Phase $\omega_{i}$; unloading

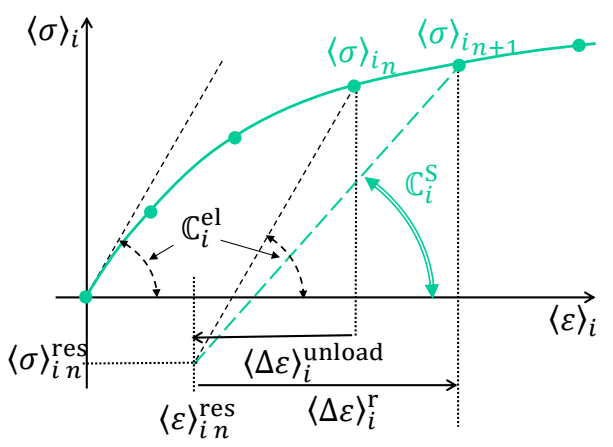

(d) Phase $\omega_{i}$; loading

Figure 3: Definition of the LCC in the incremental-secant method for elasto-plastic composites: (a) virtual elastic unloading of the composite material with the elastic operator $\mathbb{C}_{\mathrm{M}}^{\mathrm{el}}$; (b) corresponding virtual elastic unloading of an elasto-plastic phase $\omega_{i}$ with the elastic operator $\mathbb{C}_{i}^{\mathrm{el}} ;$ (c) incremental-secant loading of the composite material from the virtually unloaded state and definition of the incremental-secant operator $\mathbb{C}_{\mathrm{M}}^{\mathrm{S}}$; and (d) corresponding incremental-secant loading of an elasto-plastic phase $\omega_{i}$ from the residual state and definition of the incremental-secant phase operator $\mathbb{C}_{i}^{S}$.

Finally, because of its intrinsic definition of the phase secant-operators, the inverse analysis of the MF-ROM model from full-field simulation results will be achieved in Section 3 in a more accurate way by formulating it in a step by step process which closely follows the incremental steps of the fullfield analysis, and by considering only the volume average stress and strain tensors of the SVEs.

Virtual elastic unloading. The virtual elastic unloading is conducted on the composite material from the configuration at time $t_{n}$ to reach a residual state so that $\boldsymbol{\sigma}_{\mathrm{M} n}^{\mathrm{res}}=0$, where the subscript "res" refers to the virtually unloaded 


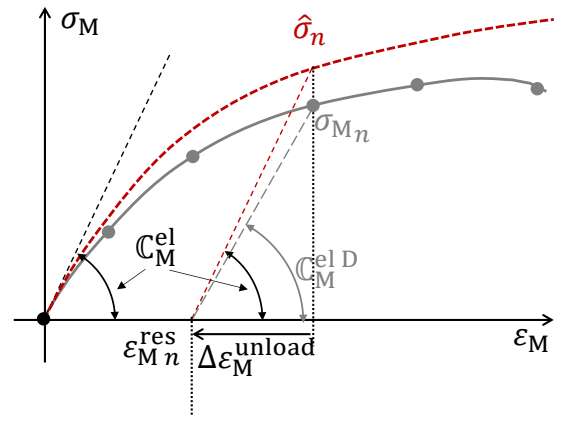

(a) Composite; unloading

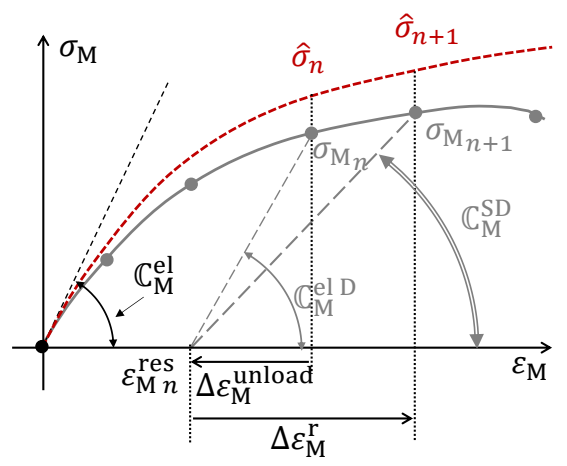

(c) Composite; loading

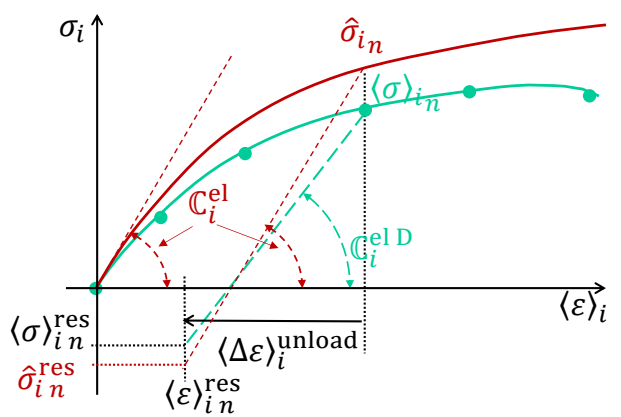

(b) Phase $\omega_{i}$; unloading

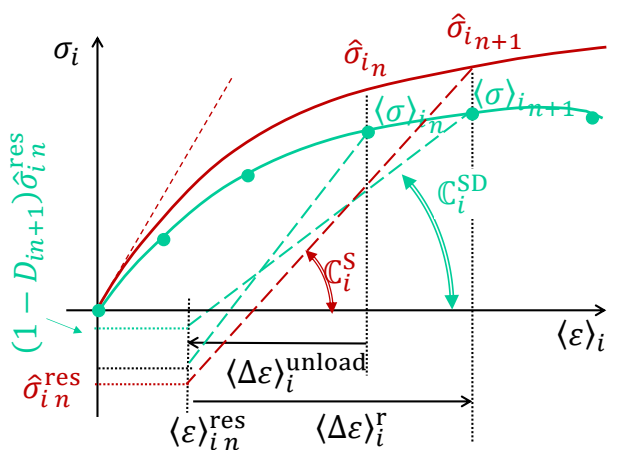

(d) Phase $\omega_{i}$; loading

Figure 4: Definition of the LCC in the incremental-secant method for damage-enhanced elasto-plastic composites: (a) virtual elastic unloading of the composite material with the elastic operator $\mathbb{C}_{\mathrm{M}}^{\mathrm{el}} \mathrm{D}$, the red dotted line corresponds to an undamaged composite and is shown for illustration purpose only; (b) corresponding virtual elastic unloading of an elasto-plastic phase $\omega_{i}$ with the elastic operator $\mathbb{C}_{i}^{\text {el D }}$, the red line corresponds to the stress-strain curve of the undamaged phase material; (c) incremental-secant loading of the composite material from the virtually unloaded state and definition of the incremental-secant operator $\mathbb{C}_{\mathrm{M}}^{\mathrm{SD}}$; and (d) corresponding incremental-secant loading of a damage-enhanced elasto-plastic phase $\omega_{i}$ from the residual undamaged stress and definition of the incremental-secant phase operator $\mathbb{C}_{i}^{S}$; the damaged incremental-secant phase operator $\mathbb{C}_{i}^{\mathrm{SD}}$ is obtained in the effective stress space.

state. We consider the case of damage-enhanced elasto-plasticity illustrated in Figs. 4(a) and 4(b) since the elasto-plastic case can be directly deduced from it.

Since this virtual unloading is elastic, the LCC is defined from the phase damaged elastic operators $\mathbb{C}_{i}^{\mathrm{el} \mathrm{D}}=\left(1-D_{i_{n}}\right) \mathbb{C}_{i}^{\mathrm{el}}$, with in the case of an isotropic 
behavior

$$
\mathbb{C}_{i}^{\mathrm{el}}=3 \kappa_{i} \mathbb{I}^{\mathrm{vol}}+2 \mu_{i} \mathbb{I}^{\mathrm{dev}}
$$

where $\kappa_{i}$ and $\mu_{i}$ are the (elastic) bulk and shear modulii of the material of phase $\omega_{i}$. When considering anisotropic elasticity for the fiber phase $\omega_{\mathrm{I}}, \mathbb{C}_{\mathrm{I}}^{\mathrm{el}}$ results from the anisotropy model.

Therefore, considering that only the matrix phase can experience damage, the set of Eqs. (27-28) is rewritten as

$$
\begin{cases}\Delta \boldsymbol{\varepsilon}_{\mathrm{M}}^{\text {unload }} & =v_{0}\langle\Delta \boldsymbol{\varepsilon}\rangle_{0}^{\text {unload }}+v_{\mathrm{I}}\langle\Delta \boldsymbol{\varepsilon}\rangle_{\mathrm{I}}^{\text {unload }} \\ 0=\boldsymbol{\sigma}_{\mathrm{M}}^{\text {res }} & =v_{0}\langle\boldsymbol{\sigma}\rangle_{0_{n}}^{\text {res }}+v_{\mathrm{I}}\langle\boldsymbol{\sigma}\rangle_{\mathrm{I}_{n}}^{\text {res }} \text { with } \\ \langle\Delta \boldsymbol{\varepsilon}\rangle_{\mathrm{I}}^{\text {unload }} & =\mathbb{B}^{\epsilon}\left(\mathrm{I}, \mathbb{C}_{0}^{\text {el D }}, \mathbb{C}_{\mathrm{I}}^{\text {el }}\right):\langle\Delta \boldsymbol{\varepsilon}\rangle_{0}^{\text {unload }} .\end{cases}
$$

This system of equations can be solved analytically using Eq. (26) rewritten as

$$
\boldsymbol{\sigma}_{\mathrm{M} n}=\mathbb{C}_{\mathrm{M}}^{\mathrm{el} \mathrm{D}}\left(\mathrm{I}, \mathbb{C}_{0}^{\mathrm{el} \mathrm{D}}, \mathbb{C}_{\mathrm{I}}^{\mathrm{el}}, v_{\mathrm{I}}\right): \Delta \varepsilon_{\mathrm{M}}^{\mathrm{unload}},
$$

where the macro-scale damaged elastic operator $\mathbb{C}_{\mathrm{M}}^{\mathrm{el}}$ reads

$$
\mathbb{C}_{\mathrm{M}}^{\mathrm{el}}=\left[v_{\mathrm{I}} \mathbb{C}_{\mathrm{I}}^{\mathrm{el}}: \mathbb{B}^{\epsilon}\left(\mathrm{I}, \mathbb{C}_{0}^{\mathrm{el} \mathrm{D}}, \mathbb{C}_{\mathrm{I}}^{\mathrm{el}}\right)+v_{0} \mathbb{C}_{0}^{\mathrm{el} \mathrm{D}}\right]:\left[v_{\mathrm{I}} \mathbb{B}^{\epsilon}\left(\mathrm{I}, \mathbb{C}_{0}^{\mathrm{el} \mathrm{D}}, \mathbb{C}_{\mathrm{I}}^{\mathrm{el}}\right)+v_{0} \mathrm{I}\right]^{-1}
$$

Although the residual stress related to the unloaded composite material vanishes, this is not the case for phases, see Fig. 4(b), which are characterized by a residual strain tensor $\langle\varepsilon\rangle_{i_{n}}^{\text {res }}=\langle\varepsilon\rangle_{i_{n}}-\langle\Delta \varepsilon\rangle_{i}^{\text {unload }}$ and a residual stress tensor $^{3}\langle\boldsymbol{\sigma}\rangle_{i_{n}}^{\text {res }}$ obtained from the resolution of the set of Eqs. (30). The undamaged stress tensors in the configuration at time $t_{n}$ and in the residual configuration follow from

$$
\hat{\boldsymbol{\sigma}}_{i_{n}}=\frac{\langle\boldsymbol{\sigma}\rangle_{i_{n}}}{\left(1-D_{i_{n}}\right)} \quad \text { and } \hat{\boldsymbol{\sigma}}_{i_{n}}^{\mathrm{res}}=\frac{\langle\boldsymbol{\sigma}\rangle_{i_{n}}^{\mathrm{res}}}{\left(1-D_{i_{n}}\right)} .
$$

Incremental-secant loading. The secant linearization of the non-linear composite material is carried out in the time interval $\left[t_{n}, t_{n+1}\right]$ from the residual state with the strain increment $\Delta \varepsilon_{\mathrm{M}}^{\mathrm{r}}$ defined such that

$$
\varepsilon_{\mathrm{M}_{n+1}}=\varepsilon_{\mathrm{M}_{n}}^{\mathrm{res}}+\Delta \varepsilon_{\mathrm{M}}^{\mathrm{r}}
$$

\footnotetext{
${ }^{3}$ For completeness, when considering first statistical moments only, it has been shown that the predictive capabilities of the incremental-secant method are improved in the case of hard inclusions when the residual stress in the matrix phase $\boldsymbol{\sigma}_{0_{n}}^{\text {res }}$ is canceled $[79,83]$. However since in this work we use the MFH as a ROM, we always keep this residual.
} 
see Fig. 4(c), where $\varepsilon_{\mathrm{M}_{n+1}}$ is known from the macro-scale BVP.

Similarly, the phase strain increments $\langle\Delta \varepsilon\rangle_{i}^{\mathrm{r}}$ are defined such that

$$
\langle\varepsilon\rangle_{i_{n+1}}=\varepsilon_{i_{n}}^{\mathrm{res}}+\langle\Delta \varepsilon\rangle_{i}^{\mathrm{r}},
$$

as illustrated in Fig. 4(d). In each phase, a incremental-secant operator is also defined in the undamaged stress state space with

$$
\hat{\boldsymbol{\sigma}}_{i_{n+1}}=\hat{\boldsymbol{\sigma}}_{i_{n}}^{\mathrm{res}}+\Delta \hat{\boldsymbol{\sigma}}_{i}^{\mathrm{r}}, \text { and } \Delta \hat{\boldsymbol{\sigma}}_{i}^{\mathrm{r}}=\mathbb{C}^{\mathrm{S}}:\langle\Delta \varepsilon\rangle_{i}^{\mathrm{r}} .
$$

The apparent stress tensor then follows as

$$
\langle\boldsymbol{\sigma}\rangle_{i_{n+1}}=\left(1-D_{i_{n+1}}\right) \hat{\boldsymbol{\sigma}}_{i_{n}}^{\mathrm{res}}+\mathbb{C}_{i}^{\mathrm{SD}}:\langle\Delta \boldsymbol{\varepsilon}\rangle_{i}^{\mathrm{r}} \text {, with } \mathbb{C}_{i}^{\mathrm{SD}}=\left(1-D_{i_{n+1}}\right) \mathbb{C}_{i}^{\mathrm{S}} .
$$

Therefore, the LCC is defined from the phase residual states using the incremental-secant operators $\mathbb{C}_{i}^{S D}$. Considering that only the matrix phase can experience damage, the set of Eqs. (27-28) is thus rewritten as

$$
\left\{\begin{array}{l}
\Delta \varepsilon_{\mathrm{M}}^{\mathrm{r}}=v_{0}\langle\Delta \boldsymbol{\varepsilon}\rangle_{0}^{\mathrm{r}}+v_{\mathrm{I}}\langle\Delta \boldsymbol{\varepsilon}\rangle_{\mathrm{I}}^{\mathrm{r}} \text { and } \\
\boldsymbol{\sigma}_{\mathrm{M}_{n+1}}=v_{0}\langle\boldsymbol{\sigma}\rangle_{0_{n+1}}+v_{\mathrm{I}}\langle\boldsymbol{\sigma}\rangle_{\mathrm{I}_{n+1}} \\
\langle\Delta \boldsymbol{\varepsilon}\rangle_{\mathrm{I}}^{\mathrm{r}}=\mathbb{B}^{\epsilon}\left(\mathrm{I}, \mathbb{C}_{0}^{\mathrm{SD}}, \mathbb{C}_{\mathrm{I}}^{\mathrm{S}}\right):\langle\Delta \boldsymbol{\varepsilon}\rangle_{0}^{\mathrm{r}}
\end{array}\right.
$$

where the (damaged) incremental-secant operators $\mathbb{C}_{0}^{\mathrm{SD}}$ of the matrix phase and $\mathbb{C}_{\mathrm{I}}^{\mathrm{S}}$ of the inclusions phase are uniform by construction and do not necessarily correspond to the volume averages of their micro-scale counter parts. The resolution of the MFH equations (38) follows an iterative process detailed in [80] and summarized in Appendix C.

Finally, the incremental-secant form of Eq. (26) reads

$$
\boldsymbol{\sigma}_{\mathrm{M}_{n+1}}=\Delta \boldsymbol{\sigma}_{\mathrm{M}}^{\mathrm{r}}=\mathbb{C}_{\mathrm{M}}^{\mathrm{S}}\left(\mathrm{I}, \mathbb{C}_{0}^{\mathrm{SD}}, \mathbb{C}_{\mathrm{I}}^{\mathrm{S}}, v_{\mathrm{I}}\right): \Delta \varepsilon_{\mathrm{M}}^{\mathrm{r}}
$$

where the macro-scale incremental-secant operator $\mathbb{C}_{\mathrm{M}}^{\mathrm{S}}$ reads

$$
\mathbb{C}_{\mathrm{M}}^{\mathrm{S}}=\left[v_{\mathrm{I}} \mathbb{C}_{\mathrm{I}}^{\mathrm{S}}: \mathbb{B}^{\epsilon}\left(\mathrm{I}, \mathbb{C}_{0}^{\mathrm{SD}}, \mathbb{C}_{\mathrm{I}}^{\mathrm{S}}\right)+v_{0} \mathbb{C}_{0}^{\mathrm{SD}}\right]:\left[v_{\mathrm{I}} \mathbb{B}^{\epsilon}\left(\mathrm{I}, \mathbb{C}_{0}^{\mathrm{SD}}, \mathbb{C}_{\mathrm{I}}^{\mathrm{S}}\right)+v_{0} \mathbb{I}\right]^{-1}
$$

Evaluation of the phase incremental-secant operator $\mathbb{C}_{i}^{\mathrm{SD}}$. During an elastic response, which is always the case for an anisotropic elastic phase, the incremental-secant operator $\mathbb{C}_{i}^{S}$ of phase $\omega_{i}$ equals its elastic tensor $\mathbb{C}_{i}^{\mathrm{el}}(29)$.

During a $J_{2}$-elasto-plastic flow in the phase, the linear comparison material is defined as isotropic elastic by assumption. It was shown in [79] that 
this isotropicity is naturally obtained with the incremental-secant approach by considering a return mapping pointing to the residual stress tensor, i.e. a plastic flow direction satisfying

$$
\boldsymbol{N}_{i}=\frac{3}{2} \frac{\mathbb{I}^{\mathrm{dev}}: \mathbb{C}_{i}^{\mathrm{el}}:\langle\Delta \varepsilon\rangle_{i}^{\mathrm{r}}}{\left(\mathbb{C}_{i}^{\mathrm{el}}:\langle\Delta \varepsilon\rangle_{i}^{\mathrm{r}}\right)^{\mathrm{eq}}}=\frac{3}{2} \frac{\mathbb{I}^{\mathrm{dev}}: \mathbb{C}_{i}^{\mathrm{S}}:\langle\Delta \varepsilon\rangle_{i}^{\mathrm{r}}}{\left(\mathbb{C}_{i}^{\mathrm{S}}:\langle\Delta \varepsilon\rangle_{i}^{\mathrm{r}}\right)^{\mathrm{eq}}},
$$

where $\left(\mathbb{C}_{i}^{\mathrm{el}}:\langle\Delta \varepsilon\rangle_{i}^{\mathrm{r}}\right)^{\mathrm{eq}}$ holds for the equivalent von Mises stress operator. Expression (41) avoids the isotropic projection of the LCC operator usually required with affine and incremental-tangent $\mathrm{MFH}[82,77,84]$. When considering the return mapping (41) for a single phase material, since the virtual elastic unloading is aligned with the elastic strain at time $t_{n}$, one can show that $\boldsymbol{N}=\frac{3}{2} \frac{\mathbb{I}^{\mathrm{dev}}: \mathbb{C}^{\mathrm{el}}: \Delta \boldsymbol{\varepsilon}^{\mathrm{r}}}{\left(\mathbb{C}^{\mathrm{el}}: \Delta \boldsymbol{\varepsilon}^{\mathrm{r}}\right)^{\mathrm{eq}}}=\frac{3}{2} \frac{\mathbb{I}^{\mathrm{dev}}:\left(\hat{\boldsymbol{\sigma}}_{n}+\mathcal{O}\left(\Delta \boldsymbol{\varepsilon}^{\mathrm{f}}\right)\right)}{\left(\hat{\boldsymbol{\sigma}}_{n}+\mathcal{O}\left(\Delta \varepsilon^{\mathrm{f}}\right)\right)^{\mathrm{eq}}}$ with $\Delta \boldsymbol{\varepsilon}^{\mathrm{f}}=\varepsilon_{n+1}-\varepsilon_{n}$. After development in a Taylor's series, one has $\boldsymbol{N}=\frac{3}{2} \frac{\frac{\pi}{\mathrm{dev}}^{\mathrm{d}} \cdot \hat{\boldsymbol{\sigma}}_{n}}{\left(\hat{\boldsymbol{\sigma}}_{n}\right)^{\mathrm{eq}}}+\mathcal{O}\left(\Delta \varepsilon^{\mathrm{f}}\right)$ showing that the return direction is a first order approximation of the radial direction. When considering a two-phase elastic material, unless the fiber volume fraction tends to 0 , the average virtual elastic unloading $\Delta \varepsilon_{i}^{\text {unload }}$ is not necessarily aligned with the $\mathbb{C}_{i}^{\mathrm{el}^{-1}}: \hat{\boldsymbol{\sigma}}_{i}$, as it can be seen by combining Eqs. (30-32), and the return mapping applied on the homogenized phase behavior is not necessarily normal to the homogenized undamaged stress tensor. However, when developing a MFH scheme, one has to make assumptions on the phases behaviors which are constructed as being uniform. Only the stress tensor is strictly a volume average of the phase distribution, but locally the plastic flow is not uniformly distributed and thus the direction of the plastic flow constructed to be uniform does not have to be normal to the yield surface built from $\hat{\boldsymbol{\sigma}}_{n+1}$ (this would actually be another assumption since the internal variables cannot be volume averaged).

Therefore, the incremental-secant operator admits the following decomposition:

$$
\mathbb{C}_{i}^{\mathrm{S}}=3 \kappa_{i} \mathbb{I}^{\mathrm{vol}}+2 \mu_{i}^{\mathrm{s}} \mathbb{I}^{\mathrm{dev}}
$$

where $\mu_{i}^{\mathrm{s}}$ is the shear modulus of the virtual elastic material. For this nonlinear phase, this virtual elastic shear modulus $\mu_{i}^{\mathrm{s}}$ can be computed through

$$
\mu_{i}^{\mathrm{s}}=\mu_{i}\left(1-\frac{\Delta p_{i}}{\left(\langle\Delta \varepsilon\rangle_{i}^{\mathrm{r}}\right)^{\mathrm{eq}}}\right)
$$


where $\left(\left\langle\Delta \varepsilon^{\mathrm{r}}\right\rangle\right)_{i}^{\mathrm{eq}}$ is the equivalent increment strain, and $\Delta p_{i}$ is the equivalent accumulated plastic strain which is evaluated through the von Mises criterion (8) and hardening law $R_{i}\left(p_{i}\right)$.

With the first statistical moments formula [79], the equivalent von Mises stresses and equivalent increment strains, corresponding to the averaged stress and strain tensors in phases $\omega_{i}$, are evaluated as

$$
\begin{aligned}
\sigma_{i}^{\mathrm{eq}} & =\sqrt{\frac{3}{2}\langle\boldsymbol{\sigma}\rangle_{\omega_{i}}: \mathbb{I}^{\mathrm{dev}}:\langle\boldsymbol{\sigma}\rangle_{\omega_{i}}} \\
\text { and } \Delta \varepsilon_{i}^{\mathrm{eq}} & =\sqrt{\frac{2}{3}\langle\Delta \varepsilon\rangle_{\omega_{i}}: \mathbb{I}^{\mathrm{dev}}:\langle\Delta \varepsilon\rangle_{\omega_{i}}} .
\end{aligned}
$$

The terms $\sigma_{i}^{\text {eq }}$ and $\Delta \varepsilon_{i}^{\text {eq }}$ can also be evaluated with the second statistical moments formula, see the work in [83] for details.

\section{Stochastic MF-ROM for non-linear composites}

Homogenized material responses can be evaluated by the M-T method following Section 2.2.2 from given micro-structure descriptions and phases material properties. In classical $\mathrm{MFH}$ applications, these micro-structure properties are identified in a unique way, hence leading to deterministic homogenized properties. In this work, in order to reproduce the uncertainties observed by conducting computational homogenization on different SVE realizations following the method described in Section 2.2.1, the micro-structure descriptions and phases material properties of the MFH model correspond to a random vector whose observations are obtained by an inverse analysis from the behaviors evaluated on SVE realizations by computational homogenization.

The MFH and the computational homogenization methods have identical definitions of the homogenized strain tensor increment $\Delta \varepsilon_{\mathrm{M}}$ and stress tensor $\boldsymbol{\sigma}_{\mathrm{M}}$, which are respectively the volume averaged values of their micro-scale counterparts $\Delta \varepsilon_{\mathrm{m}}$ and $\boldsymbol{\sigma}_{\mathrm{m}}$, see Eqs. (11) and (28). Besides, computational homogenization satisfies the Hill-Mandel condition (12). Therefore, in order to reproduce the homogenized response obtained by computational homogenization without violating the Hill-Mandel condition, the inverse analysis should only use the homogenized stress evolution $\boldsymbol{\sigma}_{\mathrm{M}}$ in term of the homogenized strain tensor $\varepsilon_{\mathrm{M}}$ history, which is the natural output of the computational homogenization process. To this end, we build the MF-ROM by 
defining effective micro-scale properties in order to satisfy Eq. (39) at all points of the strain-stress evolution.

First the case of linear elasticity previously developed in [50] is briefly recalled. Then the non-linear elasto-plastic case and the damage-enhanced non-linear case are successively derived.

Note for completeness, that inverse identification was already combined to $\mathrm{MFH}$ in the literature. In order to determine the size of RVE, MoriTanaka MFH with incremental-tangent formula [85] was adopted in [86] to extract the effective matrix parameters with a global error minimization. Effective matrix behavior of nano-composites has been identified with the overall mechanical behavior given by molecular dynamics simulations in [87].

\subsection{Random descriptors of linear elastic composites}

The inverse MFH procedure of linear elastic composites is recalled briefly for the sake of completeness, more details are given in [50].

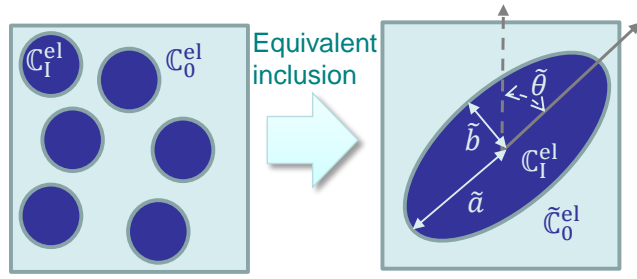

(a) Equivalent inclusion

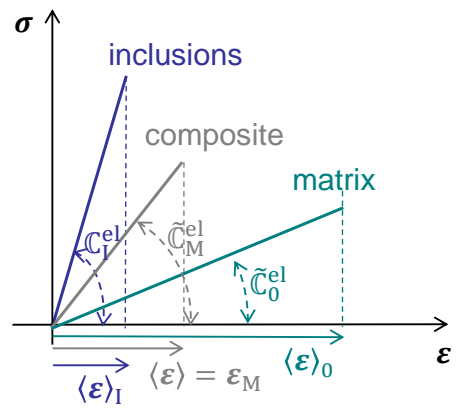

(b) Elastic MFH

Figure 5: Definition of the equivalent inclusion problem for linear elastic materials: (a) Definition of the micro-structure geometrical and phases material properties; and (b) 1D illustration of the MFH elastic response.

In order to reproduce with $\mathrm{MFH}$ the randomness observed by computational homogenization on different SVE realizations, the MFH micro-structure geometrical and phases material properties are represented by random variables whose observations are identified from an inverse analysis. In the following section, we use the notation $\tilde{\bullet}$ to indicate these effective parameters/values obtained from the inverse analysis. Therefore, in linear elasticity, we want to reproduce in $\tilde{\mathbb{C}}_{\mathrm{M}}^{\mathrm{el}}$, the meso-scale material tensor evaluated by 
the M-T method following Eq. (32), the randomness observed in $\mathbb{C}_{\mathrm{M}}^{\mathrm{el}}$, extracted by computational homogenization on different SVE realizations following Section 2.2.1. Besides, some micro-structure geometrical and phases material properties can directly be measured from the SVE realizations and do not result from the inverse identification process, in which case we do not use the $\tilde{\bullet}$ notation. In linear elasticity, the different micro-structure geometrical and phases material properties are, see Fig. 5,

- The volume fractions of inclusions $v_{\mathrm{I}}$, which can be directly measured from the SVE realizations;

- The geometrical information of the effective inclusions I, which correspond to the ellipsoid radii $\tilde{a}$ and $\tilde{b}$ for the case of UD-fiber reinforced composites;

- The orientation variable of the effective inclusion, which is a rotation angle $\tilde{\theta}$ for the case of UD-fiber reinforced composites, since the local coordinates of the effective inclusions do not generally coincide with the global coordinates of composites or of SVEs;

- The effective elasticity properties of the isotropic matrix $\tilde{\mathbb{C}}_{0}^{\mathrm{el}}\left(\tilde{E}_{0}, \tilde{\nu}_{0}\right)$, which is represented by its effective Young's modulus $\tilde{E}_{0}$ and Poisson ratio $\tilde{\nu}_{0}$;

- The effective anisotropic elasticity tensor of fibers $\mathbb{C}_{\mathrm{I}}^{\mathrm{el}}$, which can be considered to be constant for all the SVE realizations [50].

On the one hand, the observations of $v_{\text {I }}$ are obtained directly from the realizations of SVEs. Besides, for UD-fiber reinforced composites, the material response is dominated by the behavior of the matrix phase when transversely loaded and by that of the fiber phase when longitudinally loaded, the transverse properties are kept as the inclusion material properties used in the computational homogenization. However, because of the expression for UDfiber reinforced composites of the strain concentration tensor (25), the M-T model corresponds to the Voigt assumption for the longitudinal response, which is an upper bound for the homogenized property. Therefore a reduced longitudinal Young's modulus $E_{\mathrm{I}}^{\mathrm{L}}$ is identified through a simple pre-step, which reads,

$$
\min _{E_{\mathrm{I}}^{\mathrm{L}}}\left|\left[\tilde{\mathbb{C}}_{\mathrm{M}}^{\mathrm{el}}\left(\mathrm{I}, \mathbb{C}_{0}, \mathbb{C}_{\mathrm{I}}\left(E_{\mathrm{I}}^{\mathrm{L}}\right) ; v_{\mathrm{I}}\right)-\mathbb{C}_{\mathrm{M}}^{\mathrm{el}}\right]_{3333}\right|
$$


where $[\cdot]_{3333}$ indexes the entry of a fourth-order tensor along the longitudinal direction. Afterward, the effective elasticity tensor of fiber $\mathbb{C}_{\mathrm{I}}^{\mathrm{el}}$ is assumed to be constant for all the SVE realizations.

On the other hand, the observations of other random variables are extracted from an inverse MFH study, which is stated under the following optimization problem to be solved on each SVE realization

$$
\min _{\tilde{\mathrm{I}}, \tilde{\theta}, \tilde{E}_{0}, \tilde{\nu}_{0}}\left\|\tilde{\mathbb{C}}_{\mathrm{M}}^{\mathrm{el}}\left(\tilde{\mathrm{I}}, \tilde{\theta}, \tilde{\mathbb{C}}_{0}^{\mathrm{el}}\left(\tilde{E}_{0}, \tilde{\nu}_{0}\right) ; \mathbb{C}_{\mathrm{I}}^{\mathrm{el}}, v_{\mathrm{I}}\right)-\mathbb{C}_{\mathrm{M}}^{\mathrm{el}}\right\|
$$

where $\|\bullet\|$ refers to the Frobenius norm. By solving the optimization problem (47), the uncertain elastic response of the SVEs, $\mathbb{C}_{\mathrm{M}}^{\mathrm{el}}$, is represented by five random variables under the framework of $\mathrm{MFH}, \tilde{\mathbb{C}}_{\mathrm{M}}^{\mathrm{el}}\left(v_{\mathrm{I}}, \tilde{\mathrm{I}}, \tilde{\theta}, \tilde{E}_{0}, \tilde{\nu}_{0}\right)$.

\subsection{Random descriptors of non-linear elasto-plastic composites}

The inverse MFH study of non-linear composites is based on the evolution of the meso-stresses $\boldsymbol{\sigma}_{\mathrm{M} n}$ with respect to the meso-strains $\boldsymbol{\varepsilon}_{\mathrm{M} n}$ at different loading configuration times $t_{n}(n=1,2, \ldots)$, evaluated by full-field analyzes on different SVE realizations. The geometrical information $\tilde{I}, \tilde{\theta}$ of the effective inclusion as well as the effective elastic properties of the matrix phase $\tilde{\mathbb{C}}_{0}^{\mathrm{el}}\left(\tilde{E}_{0}, \tilde{\nu}_{0}\right)$ have been evaluated during the elastic response of the SVEs following the approach presented in Section 3.1, see Fig. 6(a). Therefore what remain to be identified are the effective plastic flow $\tilde{R}_{0}\left(\tilde{p}_{0}\right)$ and the effective yielding stress $\tilde{\sigma}_{Y 0}$ of the matrix phase (we assume that the inclusions remain elastic).

This non-linear inverse process is carried out in the following steps.

- During a time interval $\left[t_{n}, t_{n+1}\right], \boldsymbol{\varepsilon}_{\mathrm{M} n}, \boldsymbol{\sigma}_{\mathrm{M}_{n}}, \boldsymbol{\varepsilon}_{\mathrm{M} n+1}$ and $\boldsymbol{\sigma}_{\mathrm{M} n+1}$ are known from the full-field analysis. A virtual elastic unloading, see Fig. $6(c)$ is performed in order to satisfy

$$
\boldsymbol{\sigma}_{\mathrm{M} n}-\tilde{\mathbb{C}}_{\mathrm{M}}^{\mathrm{el}}: \Delta \varepsilon_{\mathrm{M}}^{\mathrm{unload}}=\mathbf{0} .
$$

According to Eq. (34), this defines the effective LCC using an incremental strain

$$
\Delta \varepsilon_{\mathrm{M}}^{\mathrm{r}}=\left(\varepsilon_{\mathrm{M} n+1}-\varepsilon_{\mathrm{M} n}\right)+\tilde{\mathbb{C}}_{\mathrm{M}}^{\mathrm{el}-1}: \boldsymbol{\sigma}_{\mathrm{M} n}
$$

- Then, the incremental-secant scheme (39) is rewritten as

$$
\tilde{\boldsymbol{\sigma}}_{\mathrm{M} n+1}=\tilde{\mathbb{C}}_{\mathrm{M}}^{\mathrm{S}}\left(\tilde{\mathbb{C}}_{0}^{\mathrm{S}}, \mathbb{C}_{\mathrm{I}}^{\mathrm{S}} ; \tilde{\mathrm{I}}, \tilde{\theta}, v_{\mathrm{I}}\right): \Delta \varepsilon_{\mathrm{M}}^{\mathrm{r}}
$$




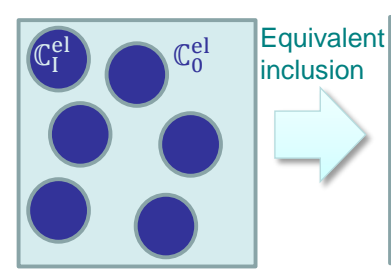

(a) Equivalent inclusion; virtual elastic unloading

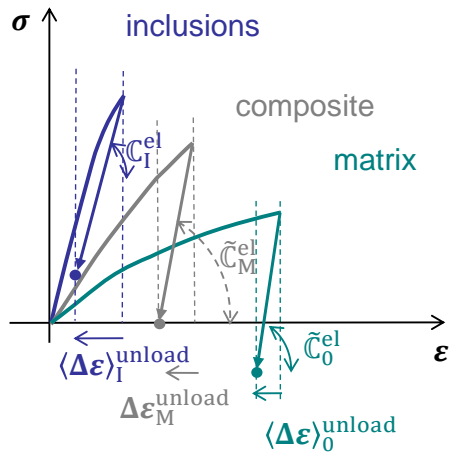

(c) Non-Linear MFH; virtual elastic unloading

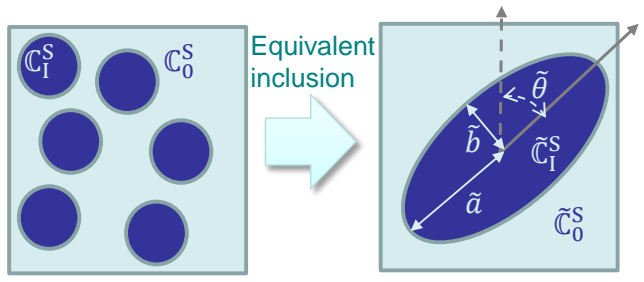

(b) Equivalent inclusion; loading

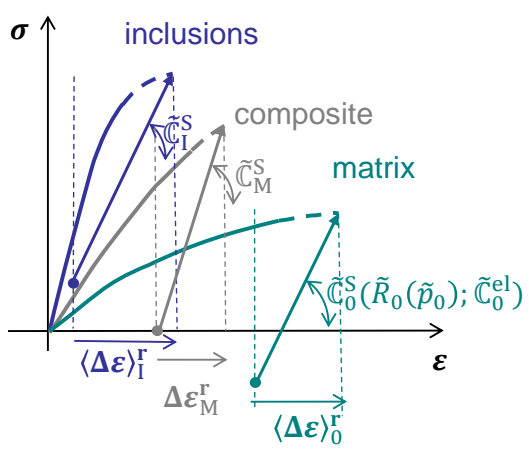

(d) Non-Linear MFH; loading

Figure 6: Definition of the equivalent inclusion problem for non-linear elasto-plastic materials: Definition of the micro-structure geometrical and phases material properties during (a) virtual elastic unloading and (b) elasto-plastic loading; and 1D illustration of the nonlinear MFH response during (c) virtual elastic unloading and (d) elasto-plastic loading.

with the expression of the tensor $\tilde{\mathbb{C}}_{\mathrm{M}}^{\mathrm{S}}$ given by Eq. (40). In order to identify the effective non-linear properties associated to the MFH process, $\widetilde{\mathbb{C}}_{\mathrm{M}}^{\mathrm{S}}$ should be evaluated so that the effective homogenized stress follows the one obtained by computational homogenization. To this end, we define the optimization problem, $\min \left\{\left\|\tilde{\boldsymbol{\sigma}}_{\mathrm{M} n+1}-\boldsymbol{\sigma}_{\mathrm{M} n+1}\right\|\right\}$, which reads, assuming that the inclusion behavior remains elastic,

$$
\min _{\tilde{\mu}_{0}^{\mathrm{S}}}\left\{\left\|\tilde{\mathbb{C}}_{\mathrm{M}}^{\mathrm{S}}\left(\tilde{\mathbb{C}}_{0}^{\mathrm{S}}\left(\tilde{\mu}_{0}^{\mathrm{S}} ; \tilde{\kappa}_{0}\right) ; \mathbb{C}_{\mathrm{I}}^{\mathrm{el}}, \tilde{\mathrm{I}}, \tilde{\theta}, v_{\mathrm{I}}\right): \Delta \boldsymbol{\varepsilon}_{\mathrm{M}}^{\mathrm{r}}-\boldsymbol{\sigma}_{\mathrm{M} n+1}\right\|\right\},
$$

where $\tilde{\mu}_{0}^{\mathrm{S}}$ is the effective virtual elastic shear modulus of the LCC's matrix material defined by Eq. (42) and $\tilde{\kappa}_{0}\left(\tilde{E}_{0}, \tilde{\nu}_{0}\right)$ is the (already identified) effective compressibility modulus of matrix phase.

- Through the resolution of the optimization problem (51) for the time in- 
terval $\left[t_{n}, t_{n+1}\right]$, the effective virtual elastic shear modulus $\tilde{\mu}_{0}^{\mathrm{S}}$ is known and the effective increment of average strain in the matrix phase, $\langle\Delta \varepsilon\rangle_{0}^{\mathrm{r}}$, can be evaluated using Eqs. (38) rewritten as

$$
\langle\Delta \varepsilon\rangle_{0}^{\mathrm{r}}=\left[v_{0} \mathbb{I}+v_{\mathrm{I}} \mathbb{B}^{\epsilon}\left(\tilde{\mathrm{I}}, \tilde{\theta}, \tilde{\mathbb{C}}_{0}^{\mathrm{S}}\left(\tilde{\mu}_{0}^{\mathrm{S}}, \tilde{\kappa}_{0}\right), \mathbb{C}_{\mathrm{I}}^{\mathrm{el}}\right)\right]^{-1}: \Delta \varepsilon_{\mathrm{M}}^{\mathrm{r}}
$$

Finally, using Eq. (43), the effective increment of accumulated plastic strain in the matrix, $\Delta \tilde{p}_{0_{n}}$, during the time interval $\left[t_{n}, t_{n+1}\right]$, is obtained following

$$
\Delta \tilde{p}_{0_{n}}=\left(\langle\Delta \varepsilon\rangle_{0}^{\mathrm{r}}\right)^{\mathrm{eq}}\left(1-\frac{\tilde{\mu}_{0}^{\mathrm{S}}}{\tilde{\mu}_{0}^{\mathrm{el}}}\right)
$$

where $\left(\langle\Delta \varepsilon\rangle_{0}^{\mathrm{r}}\right)^{\text {eq }}$ is evaluated through the first statistical moment formula, Eq. $(45)^{4}$.

- At configuration $t_{n}$, the effective von Mises stress in the matrix phase, $\tilde{\sigma}_{0_{n}}^{\text {eq }}$, is computed from Eqs. (36) and (44), and the approximated accumulated plastic strain reads

$$
\tilde{p}_{0_{n}}=\sum_{l=1}^{n} \Delta \tilde{p}_{0_{l}}
$$

Therefore, a critical accumulated plastic strain, $p_{\text {crit }}$, is defined to identify the effective initial yielding stress of the matrix phase $\tilde{\sigma}_{\mathrm{Y} 0}$. Practically, $p_{\text {crit }}$ can be any reasonably small values $\left(\right.$ e.g. $\left.\times 10^{-6} \sim \times 10^{-4}\right)$. Although the value of $p_{\text {crit }}$ will affect the resulting yielding stress, $\tilde{\sigma}_{\mathrm{Y} 0}$ and the effective parameters of the material strain hardening law, it will not have an obvious effect on the accuracy of the predicted $\mathrm{MFH}$ behavior. In this work, the critical value is set as $p_{\text {crit }}=2.0 \times 10^{-4}$. A linear interpolation is then used to evaluate the effective initial yielding stress of the matrix phase, which reads

$$
\tilde{\sigma}_{\mathrm{Y} 0}=\tilde{\sigma}_{0_{l}}^{\mathrm{eq}}+\left(p_{\text {crit }}-\tilde{p}_{0_{l}}\right) \frac{\tilde{\sigma}_{0_{l+1}}^{\mathrm{eq}}-\tilde{\sigma}_{0_{l}}^{\mathrm{eq}}}{\tilde{p}_{0_{l+1}}-\tilde{p}_{0_{l}}}, \quad \text { if } \quad \tilde{p}_{0_{l}} \leq p_{\text {crit }}<\tilde{p}_{0_{l+1}} .
$$

\footnotetext{
${ }^{4}$ This evaluation does not require to perform a volume average on the SVE
} 
Then, the recorded effective accumulated plastic strain in the matrix needs to be updated by

$$
\tilde{p}_{0_{n}} \rightarrow \tilde{p}_{0_{n}}-p_{\text {crit }}, \quad \text { for all } n \text { such that } \tilde{p}_{0_{n}}>p_{\text {crit }} .
$$

Moreover, the effective isotropic hardening stress in the matrix phase is obtained at each configuration $t_{n}$ by

$$
\tilde{R}_{0}\left(\tilde{p}_{0_{n}}\right)=\tilde{\sigma}_{0_{n}}^{\mathrm{eq}}-\tilde{\sigma}_{\mathrm{Y} 0}, \quad \text { if } \quad \tilde{p}_{0_{n}}>0 .
$$

- Finally, the effective elasto-plastic behavior $\tilde{R}_{0}\left(\tilde{p}_{0}\right)$ of the matrix phase can be represented by a proper plastic hardening model which fits the discretized hardening law $\tilde{R}_{0}\left(\tilde{p}_{0_{n}}\right)$.

In the proposed formulation, the effective parameters, including the hardening law, of the MF-ROM are identified from the full-field response through Eq. (51) only. The methodology never evaluates the volume average of internal variables such as the plastic strain on the SVEs, which would not be consistent with the Hill-Mandel condition.

\subsection{Random descriptors of non-linear damage-enhanced elasto-plastic com- posites}

In this section, a scalar damage variable $D_{0}$ is considered in the matrix phase. As for the elasto-plastic case described in the previous section, the geometrical information $\tilde{\mathrm{I}}, \tilde{\theta}$ of the effective inclusion and the effective elastic properties of the matrix phase $\tilde{\mathbb{C}}_{0}^{\text {el }}\left(\tilde{E}_{0}, \tilde{\nu}_{0}\right)$ are evaluated during the initial elastic response of the SVEs for which $D_{0}=0$, following the approach presented in Section 3.1. During the remaining direct finite element analyzes conducted on the SVEs, the damaged homogenized elastic tensor, $\mathbb{C}_{\mathrm{M} n}^{\mathrm{elD}}$ of an SVE, see Fig. $7(\mathrm{a})$, is evaluated at each time configuration $t_{n}(n=1,2, \ldots)$, together with the evolution of the meso-stress $\boldsymbol{\sigma}_{\mathrm{M} n}$ with respect to the mesostrain $\varepsilon_{\mathrm{M}_{n}}$.

In the damage-enhanced case, the effective damage evolution has to be identified through the inverse identification process before identifying the effective plastic flow $\tilde{R}_{0}(\tilde{p})$ and the effective yielding stress $\tilde{\sigma}_{Y 0}$ of the matrix phase (we assume that the inclusions remain elastic), since the latter are expressed in the undamaged stress space. Using the identified effective parameters $\tilde{\mathrm{I}}, \tilde{\theta}, \tilde{E}_{0}, \tilde{\nu}_{0}$ and $v_{\mathrm{I}}$, the effective damage $\tilde{D}_{0}$ in the matrix phase is 

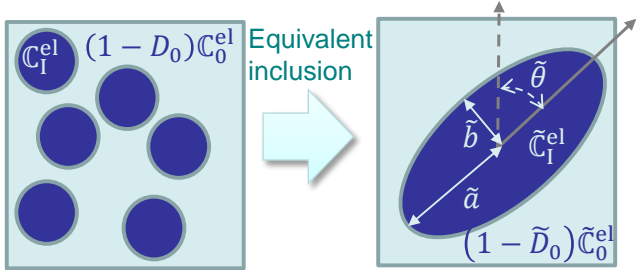

(a) Equivalent inclusion; virtual elastic unloading

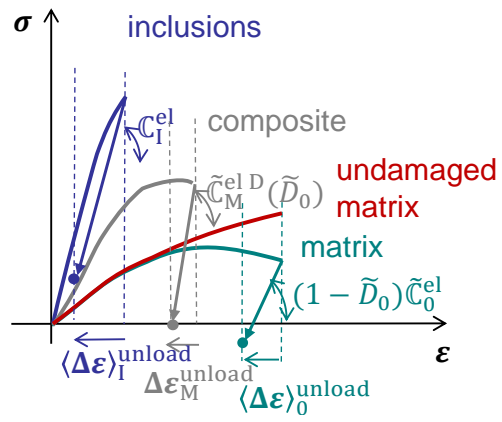

(c) Non-Linear MFH; virtual elastic unloading

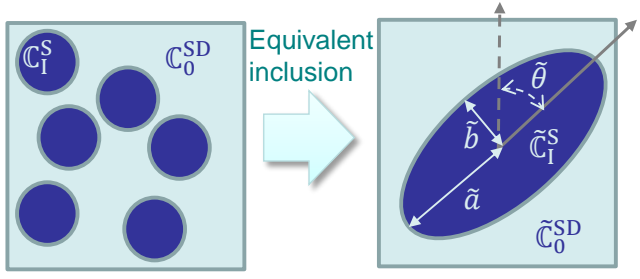

(b) Equivalent inclusion; loading

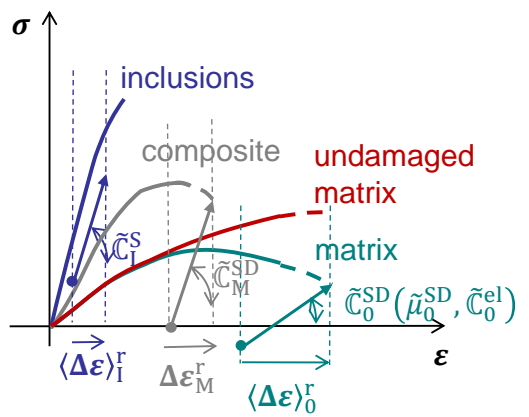

(d) Non-Linear MFH; loading

Figure 7: Definition of the equivalent inclusion problem for damage-enhanced non-linear materials: Definition of the micro-structure geometrical and phases material properties during (a) virtual elastic loading and (b) damage-enhanced elasto-plastic loading; and 1D illustration of the non-linear MFH response during (c) virtual elastic unloading and (d) damage-enhanced elasto-plastic loading.

evaluated, at each time configuration $t_{n}$, by solving the following optimization problem

$$
\min _{\tilde{D}_{0_{n}}}\left\|\tilde{\mathbb{C}}_{\mathrm{M}}^{\mathrm{el}}\left(\tilde{\mathbb{C}}_{0}^{\mathrm{el} \mathrm{D}}\left(\tilde{D}_{0_{n}}\right) ; \tilde{\mathrm{I}}, \tilde{\theta}, \tilde{E}_{0}, \tilde{\nu}_{0}, \mathbb{C}_{\mathrm{I}}^{\mathrm{el}}, v_{\mathrm{I}}\right)-\mathbb{C}_{\mathrm{M} n}^{\mathrm{el} \mathrm{D}}\right\|,
$$

where $\tilde{\mathbb{C}}_{0}^{\text {elD }}\left(\tilde{D}_{0_{n}}\right)=\left(1-\tilde{D}_{0_{n}}\right) \tilde{\mathbb{C}}_{0}^{\text {el }}\left(\tilde{E}_{0}, \tilde{\nu}_{0}\right)$ is also denoted by $\tilde{\mathbb{C}}_{0_{n}}^{\text {elD }}$.

Once the effective damage evolution $\tilde{D}_{0_{n}},(n=1,2, \ldots)$ has been obtained, the effective non-linear properties of the phases in the undamaged space can be inferred by a similar process as the one presented in Section 3.2, Eqs. (4857). However, because of the existence of damage in the matrix phase, a few modifications are needed when studying the incremental-secant formulation in the time interval $\left[t_{n}, t_{n+1}\right]$ :

- $\tilde{\mathbb{C}}_{\mathrm{M} n}^{\text {elD }}=\tilde{\mathbb{C}}_{\mathrm{M}}^{\mathrm{el}}\left(\tilde{\mathbb{C}}_{0_{n}}^{\mathrm{elD}}, \mathbb{C}_{\mathrm{I}}^{\mathrm{el}}, \tilde{\mathrm{I}}, \tilde{\theta}, v_{\mathrm{I}}\right)$ is used instead of $\tilde{\mathbb{C}}_{\mathrm{M}}^{\mathrm{el}}$ in the elastic un- 
loading step, Eqs. (48) and (49).

- The optimization problem (51) is restated in terms of $\tilde{\mu}_{0}^{\mathrm{SD}}$ as argument, and the term $\tilde{\mathbb{C}}_{\mathrm{M}}^{\mathrm{SD}}\left(\tilde{\mathbb{C}}_{0}^{\mathrm{SD}}\left(\tilde{\mu}_{0}^{\mathrm{SD}}\right) ; \mathbb{C}_{\mathrm{I}}^{\mathrm{e}}, \tilde{\mathrm{I}}, \tilde{\theta}, v_{\mathrm{I}}\right)$ substitutes to the term $\tilde{\mathbb{C}}_{\mathrm{M}}^{\mathrm{S}}\left(\tilde{\mathbb{C}}_{0}^{\mathrm{S}}\left(\tilde{\mu}_{0}^{\mathrm{S}}\right) ; \mathbb{C}_{\mathrm{I}}^{\mathrm{el}}, \tilde{\mathrm{I}}, \tilde{\theta}, v_{\mathrm{I}}\right)$, yielding

$$
\min _{\tilde{\mu}_{0}^{\mathrm{SD}}}\left\{\left\|\tilde{\mathbb{C}}_{\mathrm{M}}^{\mathrm{SD}}\left(\tilde{\mathbb{C}}_{0}^{\mathrm{SD}}\left(\tilde{\mu}_{0}^{\mathrm{SD}} ; \tilde{E}_{0}, \tilde{\nu}_{0}, \tilde{D}_{0}\right) ; \mathbb{C}_{\mathrm{I}}^{\mathrm{el}}, \tilde{\mathrm{I}}, \tilde{\theta}, v_{\mathrm{I}}\right): \Delta \boldsymbol{\varepsilon}_{\mathrm{M}}^{\mathrm{r}}-\boldsymbol{\sigma}_{\mathrm{M} n+1}\right\|\right\}
$$

Moreover, the damaged incremental-secant operator $\tilde{\mathbb{C}}_{0}^{\mathrm{SD}}\left(\tilde{\mu}_{0}^{\mathrm{SD}} ; \tilde{E}_{0}, \tilde{\nu}_{0}, \tilde{D}_{0}\right)$ is used to compute $\mathbb{B}^{\epsilon}$ in Eq. (52) instead of $\tilde{\mathbb{C}}_{0}^{\mathrm{S}}$.

- Considering the effective damage in matrix allows Eq. (53) to be rewritten as

$$
\Delta \tilde{p}_{0_{n}}=\left(\langle\Delta \varepsilon\rangle_{0}^{\mathrm{r}}\right)^{\mathrm{eq}}\left(1-\frac{\tilde{\mu}_{0}^{\mathrm{SD}}}{\tilde{\mu}_{0}^{\mathrm{elD}}}\right),
$$

with $\tilde{\mu}_{0}^{\mathrm{elD}}=\tilde{\mu}_{0}^{\mathrm{el}}\left(1-\tilde{D}_{0_{n+1}}\right)$.

- Finally, the effective equivalent undamaged stress matrix reads

$$
\tilde{\hat{\sigma}}_{0_{n+1}}^{\mathrm{eq}}=\frac{\tilde{\sigma}_{0_{n+1}}^{\mathrm{eq}}}{1-\tilde{D}_{0_{n+1}}}
$$

and is used to evaluate the isotropic hardening stress $\tilde{R}_{0}\left(\tilde{p}_{0_{n}}\right)$ in equation (57). Indeed, the plastic flow behavior is related to the equivalent value of the undamaged stress.

In order to define the governing laws of the matrix phase of the MFH model, the effective damage evolution $\tilde{D}_{0}\left(\tilde{\varepsilon}_{0}^{\mathrm{e}}, \tilde{p}_{0}\right)$ is described with a proper damage model, whose parameters can be identified through the recorded damage evolution $\tilde{D}_{0_{n}}$, and through the corresponding effective stress, $\tilde{\boldsymbol{\sigma}}_{0_{n}}$, and elastic strain, $\tilde{\varepsilon}_{0_{n}}^{\mathrm{e}}$, state in the matrix phase.

\section{Application to UD-fiber reinforced matrix composites}

In this section the inverse MFH procedure presented in Section 3 is applied to reproduce the stochastic non-linear behavior of UD-fiber reinforced matrix composites. After having described the SVEs, the stochastic inverse MFH process is applied successively to the cases of elastic fiber-reinforced elastoplastic matrix and of elastic fiber-reinforced damage-enhanced elasto-plastic matrix, with respectively 1900 and 1868 SVEs. 


\subsection{SVEs description}

\subsubsection{Micro-structure generation}

In this work it is assumed that the uncertainties result from the spatial organization of the fibers only and the material properties are considered deterministic. The SVE cross-section realizations of UD-carbon fiber reinforced composites are obtained using the methodology described in [14]. The chosen cross-section size is $25 \times 25 \mu \mathrm{m}^{2}$, for which it has been shown in [14] that the homogenized elastic properties of two neighboring SVEs are not correlated and could be represented as independent random vectors. As a result, the inclusion volume fraction within an SVE ranges from a few percents up to more than 60 percents depending on the realization. In this paper we assume that the homogenized properties could also be represented as independent random vectors in the non-linear range.

The SVEs are meshed with quadratic elements, but with a geometrical linear interpolation at the fiber/matrix interface in order to prevent the existence of concave elements. A mesh sensitivity analysis has been conducted to select a maximum mesh size of $1 \mu \mathrm{m}$.

\subsubsection{Material properties}

The UD-carbon fibers follow a transversely isotropic linear elastic constitutive material model whose material parameters are presented in Table 1.

Table 1: Material properties of the carbon fibers.

\begin{tabular}{ll}
\hline Property & Value \\
\hline Long. Young's modulus $E_{\mathrm{I}}^{\mathrm{L}}[\mathrm{GPa}]$ & 230.0 \\
Trans. Young's modulus $E_{\mathrm{I}}^{\mathrm{T}}[\mathrm{GPa}]$ & 40.0 \\
Trans. Poisson ratio $\nu_{\mathrm{I}}^{\mathrm{TT}}[-]$ & 0.20 \\
Long.-Trans. Poisson ratio $\nu_{\mathrm{I}}^{\mathrm{LT}}[-]$ & 0.256 \\
Trans. shear modulus $\mu_{\mathrm{I}}^{\mathrm{TT}}[\mathrm{GPa}]$ & 16.7 \\
Long.-Trans. shear modulus $\mu_{\mathrm{I}}^{\mathrm{LT}}[\mathrm{GPa}]$ & 24.0 \\
\hline
\end{tabular}

The matrix follows an elasto-plastic behavior model and its hardening law reads

$$
R_{0}(p)=h_{0}\left(1-e^{-m_{0} p}\right),
$$


where $h_{0}$ and $m_{0}$ are the material parameters. The elasto-plastic model of matrix material can be further enhanced by a Lemaitre [88] scalar damage setting affecting the constitutive law of matrix material following Eqs. (6-10). To avoid the mesh dependency of finite element analysis during strain softening, the damage model is formulated in a non-local form, and the evolution of the damage (7) reads

$$
\dot{D}_{0}=\left(\frac{Y}{S_{0}}\right)^{s_{0}} \dot{\chi}
$$

where $S_{0}$ and $s_{0}$ are the material parameters, and $Y$ is the strain energy release rate computed as

$$
Y=\frac{1}{2} \varepsilon^{\mathrm{el}}: \mathbb{C}_{0}^{\mathrm{el}}: \varepsilon^{\mathrm{el}}
$$

To illustrate the efficiency of the method to capture extended plasticity, a relatively ductile matrix behavior is considered as in [80]. The material parameters of the matrix material are reported in Table 2. Stress-strain curves under unidirectional tension and bi-axial loading condition of the matrix material are illustrated in Fig. 8. It can be seen that the used matrix material is quite ductile in tension compared to the epoxy used in UD-fiber reinforced matrix composites. Because of this ductility, the presented inverse procedure can be better revealed in the non-linear range than with a brittle matrix material.

Table 2: Material properties of the matrix.

\begin{tabular}{ll}
\hline Property & Value \\
\hline Young's modulus $E_{0}[\mathrm{GPa}]$ & 3.2 \\
Poisson ratio $\nu_{0}[-]$ & 0.3 \\
Initial yield stress $\sigma_{\mathrm{Y} 0}[\mathrm{MPa}]$ & 25.0 \\
Hardening modulus $h_{0}[\mathrm{MPa}]$ & 73.0 \\
Hardening exponent $m_{0}[-]$ & 60.0 \\
Damage critical energy release $S_{0}[\mathrm{Mpa}]$ & 2.0 \\
Damage exponent $s_{0}[-]$ & 0.5 \\
Damage critical plastic strain $p_{C_{0}}[-]$ & 0.0 \\
Characteristic length $l_{0}[\mathrm{~mm}]$ & $\sqrt{2}$ \\
\hline
\end{tabular}




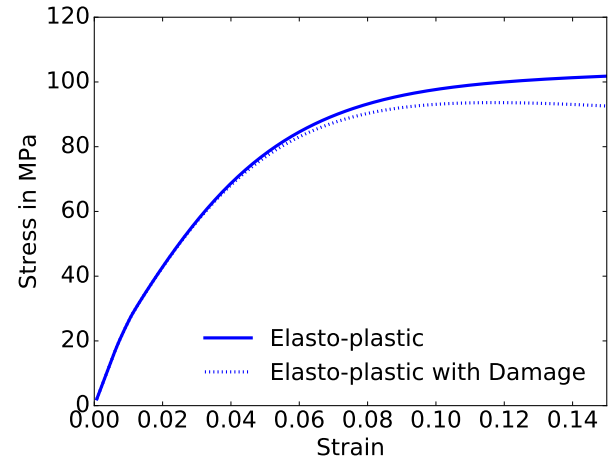

(a) Uni-axial tension

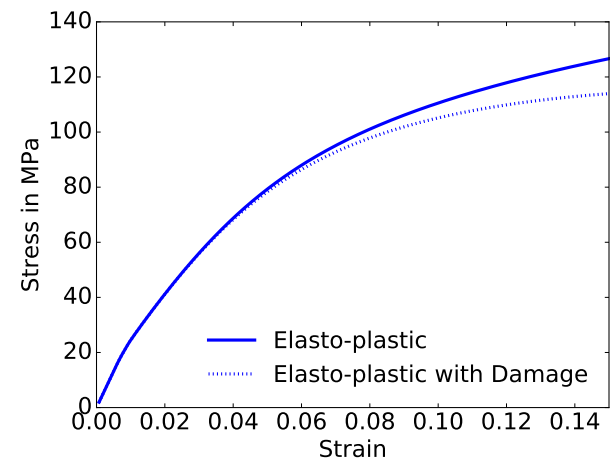

(b) Bi-axial loading

Figure 8: Stress-strain curve, $\sigma_{11}-\varepsilon_{11}$, of the matrix material whose parameters are reported in Table 2, under different boundary conditions: (a) Uni-axial tension with only $\sigma_{11} \neq 0$; (b) Bi-axial loading, with $\varepsilon_{22}=-0.5 \varepsilon_{11}$ and $\varepsilon_{33}=\varepsilon_{12}=\varepsilon_{23}=\varepsilon_{31}=0.0$.

\subsubsection{The loading conditions on the SVEs}

A loading condition is defined and used in the direct finite element analyzes performed on the SVEs to obtain the reference macro-responses. The loading condition is defined by the macro-strain $\varepsilon_{\mathrm{M}}$ which reads

$$
\varepsilon_{\mathrm{M} 11} \in[0 . ; 0.07], \varepsilon_{\mathrm{M} 22}=-0.5 \varepsilon_{\mathrm{M} 11} \text { and } \varepsilon_{\mathrm{M} 33}=\varepsilon_{\mathrm{M} 12}=\varepsilon_{\mathrm{M} 23}=\varepsilon_{\mathrm{M} 31}=0 \text {. }
$$

This loading condition is close to the state of UD-fiber reinforced composites under transverse loading with an extra strain constraint along the thickness direction of a ply.

Besides, PBCs (19) are applied on the SVE boundary. Even in the context of damage, the periodic boundary conditions capture the SVE response with accuracy since the final failure stage is not studied. We refer to the work [71] for a more complete analysis of the boundary condition effect on the failure study of UD composite SVEs.

Later, the same loading condition (65) will be applied during the MFH with the effective phases material properties obtained from the inverse MFH procedure. Since these boundary conditions do not imply a kinematic unknown, they fasten the identification process.

\subsection{Stochastic inverse MFH process for elastic fiber-reinforced elasto-plastic matrix}

According to the previous study in [50], the uncertainties can be modeled by inferring an effective inclusion geometry and an effective matrix behavior, 
while the effective properties of the carbon fibers can be considered constant for all the SVEs. In particular, the transverse-related material properties are the original ones used during the computational homogenization and presented in Table 1, while the longitudinal Young's modulus is inferred from Eq. (46) leading to $\tilde{E}_{\mathrm{L}}=222.1 \mathrm{GPa}$. Therefore, the inverse MFH procedure consists in evaluating the micro-structure effective inclusion $\tilde{\mathrm{I}}, \tilde{\theta}$ and the effective matrix properties. The micro-structure effective inclusion $\tilde{\mathrm{I}}, \tilde{\theta}$ and the effective matrix elastic properties $\tilde{E}_{0}, \tilde{\nu}_{0}$ are inferred from Eq. (47), and the results have been extensively presented in [50]. The identification of the effective matrix hardening law is discussed here after.

\subsubsection{Identification of the effective matrix isotropic hardening law}

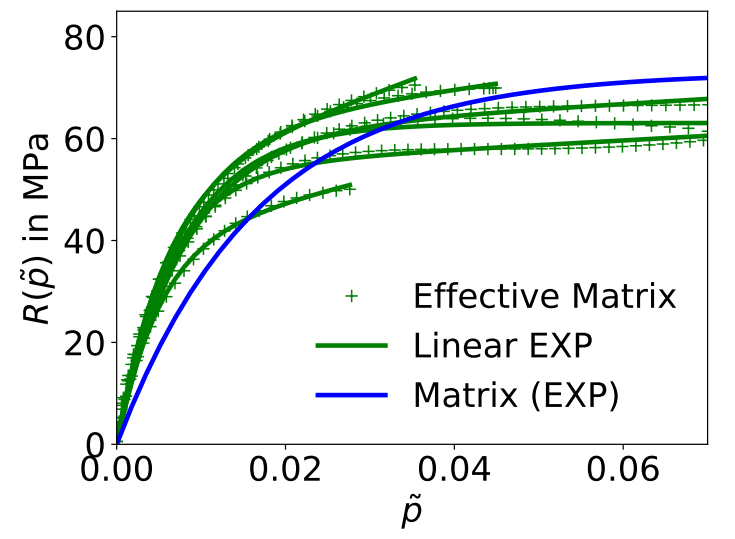

Figure 9: Isotropic hardening behavior of the the matrix material: "Matrix (EXP)" corresponds to the hardening law (62) used in the SVE direct simulations; "Effective matrix" corresponds to the effective curves obtained from the inverse identification process; "Linear EXP" corresponds to the effective behavior curve-fitted following Eq. (66).

The evolution of the isotropic hardening stress $\tilde{R}_{0}\left(\tilde{p}_{0}\right)$ associated to the effective accumulated plastic strain $\tilde{p}_{0}$ is extracted from the direct finite element analyzes, according to the inverse procedure presented in Section 3.2, and the results of a few randomly picked SVEs are presented in Fig. 9. The behavior of a pure matrix following the material parameter reported in Table 2 is also presented for comparison purpose. The figure shows that the exponential hardening law of pure matrix, Eq. (62), cannot fit the behavior of the effective matrix for all the realizations. Therefore, a linear exponential hardening law, which reads

$$
\tilde{R}_{0}\left(\tilde{p}_{0}\right)=k_{1_{0}} \tilde{p}_{0}+k_{2_{0}}\left(1-e^{-m_{0} \tilde{p}_{0}}\right),
$$


is adopted to model the behavior of the effective matrix. Compared to an exponential hardening law, the linear exponential hardening law is enriched with a linear hardening term so that the exponential hardening law (62) can be recovered by the linear exponential hardening law (66) with $k_{1_{0}}=0$.

The strain hardening parameters, $\tilde{k}_{1_{0}}, \tilde{k}_{2_{0}}$ and $\tilde{m}_{0}$ of the linear exponential hardening law of the effective matrix obtained using a standard "curve - fit" function of the Scipy library are illustrated in Fig. 9, under the label "Linear EXP".

\subsubsection{Assessment of the elasto-plastic MF-ROM}

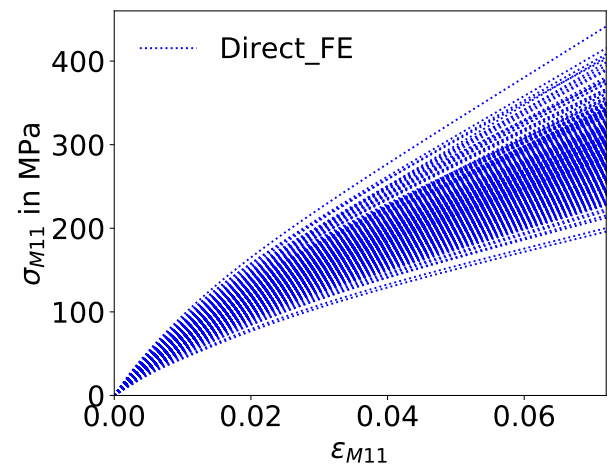

(a)

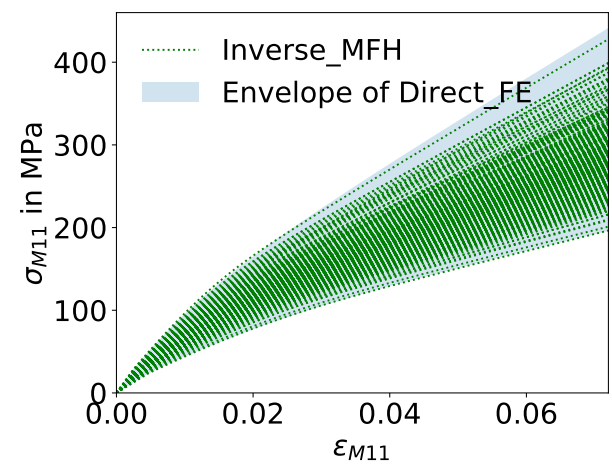

(b)

Figure 10: Homogenized responses $\sigma_{\mathrm{M} 11}-\varepsilon_{\mathrm{M} 11}$ of elasto-plastic SVEs obtained from: (a) Direct finite element analyzes; and (b) MF-ROM with effective material properties.

The responses of 750 SVEs obtained from direct finite element analyzes are plotted in Fig. 10(a) in terms of $\sigma_{\mathrm{M} 11}-\varepsilon_{\mathrm{M} 11}$ curves. The corresponding results predicted by the MF-ROM with the identified effective material properties are presented in Fig. 10(b) for comparison purpose. It can be seen that the MF-ROM reproduces well the stochastic macro responses $\sigma_{\text {M11 }}$ of the SVEs. For all the comparison results presented in Fig. 10, the maximum relative error in the stress $\sigma_{\mathrm{M} 11}$ is found to be lower than $3.5 \%$; the higher relative errors are found for the SVEs with the higher fiber volume fractions (e.g. $v_{\mathrm{I}}>60 \%$ ). For the SVEs with lower fiber volume fractions, the inverse $\mathrm{MFH}$ can provide rather accurate results, e.g. the lowest curves in Figs. 10 (a) and 10(b) refer to an SVE with a fiber volume fraction $v_{\mathrm{I}}=25 \%$, for which the relative error of MF-ROM is lower than 1.0\%. Furthermore, the statistical characteristic of SVEs macro responses is well preserved. This fea- 
ture is crucial for the future application of MF-ROM to achieve the stochastic analysis at a higher scale.

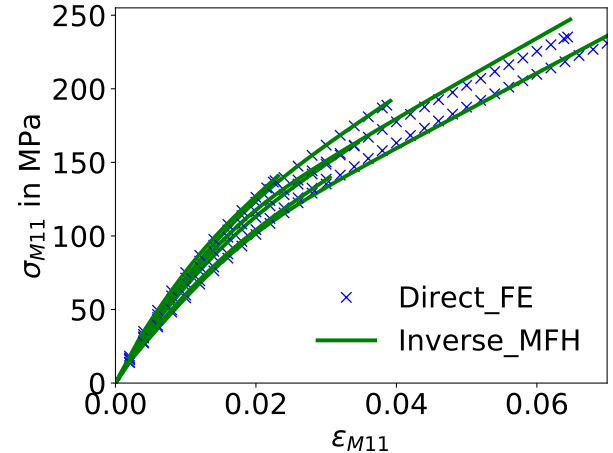

(a)

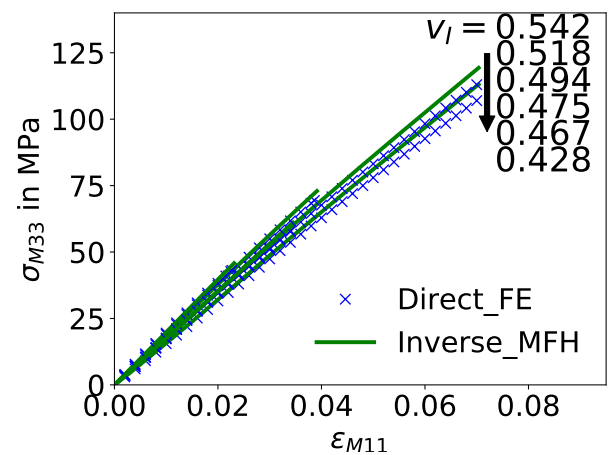

(c)

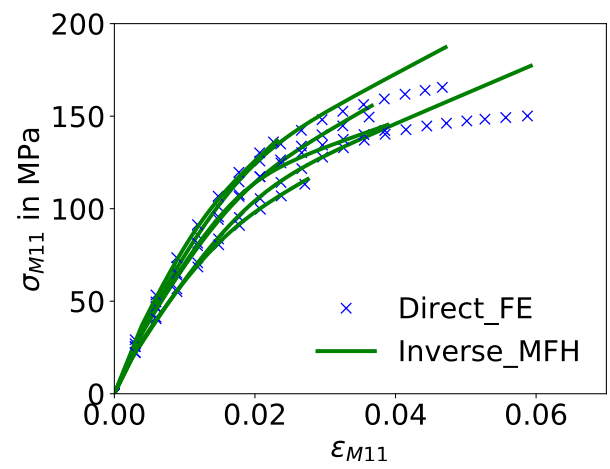

(e)

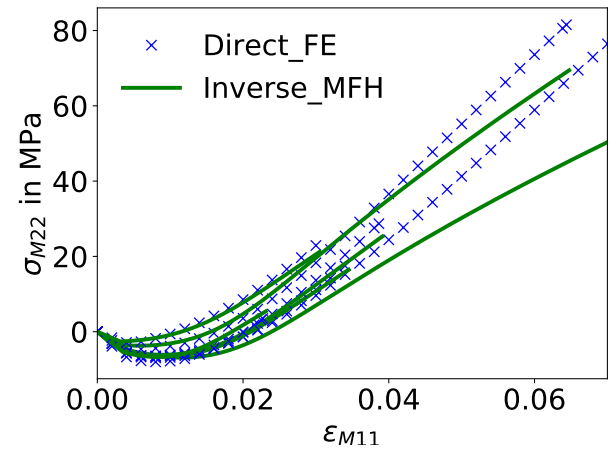

(b)

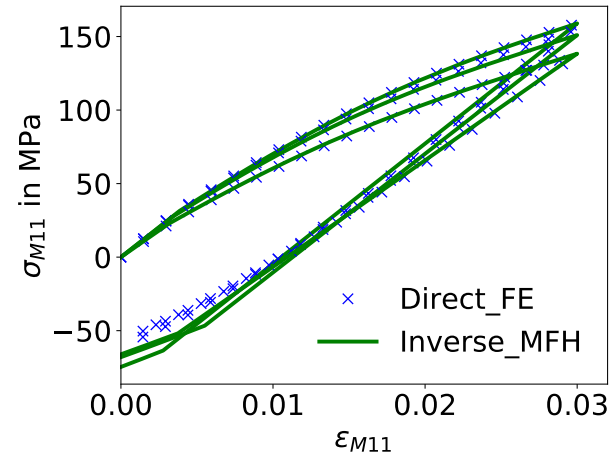

(d)

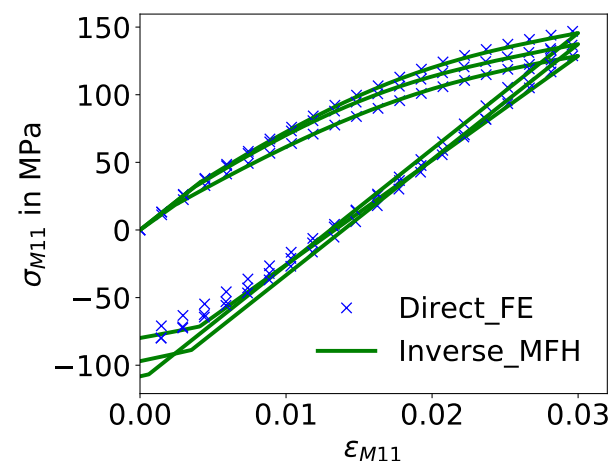

(f)

Figure 11: Homogenized response of elastic-plastic SVEs: (a)-(d) Under boundary conditions (65); and (e)-(f) Under boundary condition (67). 
A more detailed comparison of the stress evolution is presented in Fig. 11 for a few randomly picked SVEs. It can be seen that the homogenized stress components $\sigma_{\mathrm{M} 11}$ and $\sigma_{\mathrm{M} 33}$ obtained with the MF-ROM with the effective materials properties agree well with that obtained from the direct finite element analysis, see Figs. 11(a) and 11(c); Besides the trend of $\sigma_{\mathrm{M} 22}$ is well preserved as compared to the results of the finite element simulations, see Fig. 11(b). Finally, loading-unloading cycles are well captured by the MF-ROM up to the re-hardening stage during reverse plasticity, as it can be seen on Fig. 11(d).

In order to assess the effect of the loading conditions, a transverse tension, with the boundary condition specified by

$$
\varepsilon_{\mathrm{M} 33}=\varepsilon_{\mathrm{M} 12}=\varepsilon_{\mathrm{M} 23}=\varepsilon_{\mathrm{M} 31}=0 \quad \text { and } \quad \sigma_{\mathrm{M} 22}=0 \text {, }
$$

is applied on the same SVEs. The resulting $\sigma_{\mathrm{M} 11}$ predicted by the MF-ROM are compared to that of the direct finite element analysis in Fig. 11(e). Although the loading condition applied for this comparison is different from the loading condition (65) that is used for the inverse study, obvious disagreements between the finite element results and the MF-ROM predictions only appear at the later stage of the loading. However, loading-unloading cycles are well captured by the method, at the exception of the re-hardening occurring during reverse plasticity, as seen in Fig. 11(f). This indicates that using MFH with the effective phases material properties from the inverse study to reproduce the non-linear behaviors of SVEs is valid in a low to moderate strain region. However, when high strain zones develop within the SVEs with the increase of the homogenized-stain, $\varepsilon_{\mathrm{M}}$, the accuracy of the MF-ROM becomes limited.

\subsection{Stochastic inverse MFH process for elastic fiber-reinforced damage-en- hanced elasto-plastic matrix}

In the matrix damage-enhanced elasto-plastic case, the MF-ROM random variables to be identified are

- The micro-structural geometrical parameters $\tilde{\mathrm{I}}, \tilde{\theta}$ and $v_{\mathrm{I}}$, and the effective matrix elastic properties $\tilde{E}_{0}, \tilde{\nu}_{0}$ that are inferred from Eq. (47);

- The effective matrix yielding stress and hardening law (66) parameters $\tilde{\sigma}_{\mathrm{Y} 0}, \tilde{k}_{1_{0}}, \tilde{k}_{2_{0}}, \tilde{m}_{0}$ that are identified from the effective matrix undamaged stress evolution $\tilde{\boldsymbol{\sigma}}_{0}$ as detailed in Section 3.3, and which extraction 
does not differ from what had been presented in the previous subsection;

- The effective damage law (63) parameters $\tilde{S}_{0}$ and $\tilde{s}_{0}$ whose extraction is detailed here below.

\subsubsection{Identification of the effective matrix damage evolution law}

Although in the direct finite element analysis the damage variable of the matrix possesses a non-local form, in the inverse $\mathrm{MFH}$ analysis, the effective damage, which is associated to (but which is not rigorously) the volume averaged degradation of the matrix material, is written in its local form

$$
\dot{\tilde{D}}_{0}= \begin{cases}0, & \text { if } \tilde{p}_{0} \leqslant p_{C_{0}} \\ \left(\frac{Y}{S_{0}}\right)^{s_{0}} \dot{\tilde{p}}_{0}, & \text { if } \tilde{p}_{0}>p_{C_{0}},\end{cases}
$$

where $\tilde{D}_{0}$ and $\tilde{p}_{0}$ are respectively the effective matrix damage and accumulated plastic strain. We keep $p_{C_{0}}=0$, and two random variables, $\tilde{S}_{0}$ and $\tilde{s}_{0}$, are thus added to describe the effective properties of the matrix.

\subsubsection{Assessment of the damage-enhanced elasto-plastic MF-ROM}

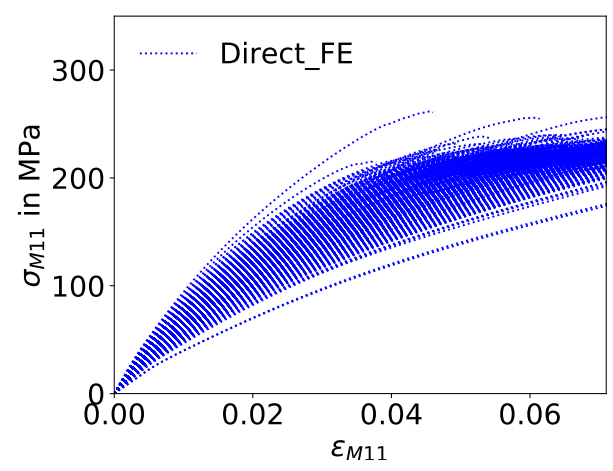

(a)

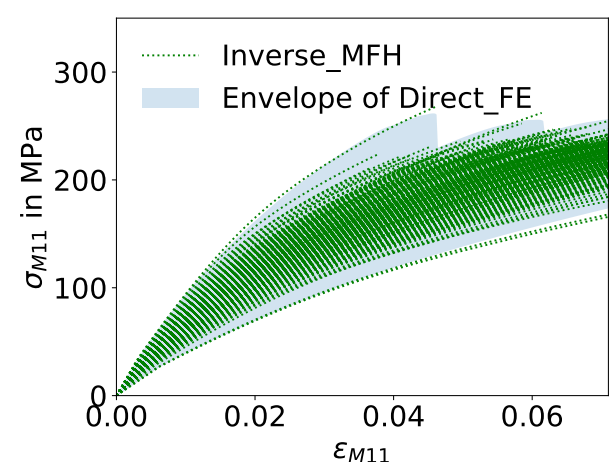

(b)

Figure 12: Homogenized responses $\sigma_{\mathrm{M} 11}-\varepsilon_{\mathrm{M} 11}$ of damage-enhanced elasto-plastic SVEs obtained from: (a) Direct finite element analyzes; and (b) MF-ROM with effective material properties.

The macro responses of 750 SVEs obtained by direct finite element analyzes are plotted in Fig. 12(a) for $\sigma_{\mathrm{M} 11}-\varepsilon_{\mathrm{M} 11}$ for boundary conditions (65). The corresponding results obtained by the MF-ROM with the effective 
phases materials properties identified following the methodology reported in Section 3.3 are presented in Fig. 12(b). Since a damage variable is applied in the matrix phase, the damage induced strain softening can be seen in the macro responses of some SVEs, see Fig. 12(a). However, for the homogenized responses $\sigma_{\mathrm{M} 11}$ reproduced by the MF-ROM, the damage induced strain softening can only be seen for a few SVEs, see Fig. 12(b). Comparing Fig. 12 to Fig. 10 shows that the MF-ROM is less accurate in the case of damage-enhanced elasto-plasticity. For some severe cases, the maximum relative error, before reaching the softening stage, is around $6 \%$ in term of the stress $\sigma_{\mathrm{M} 11}$. The statistical characteristics of SVEs homogenized responses is only preserved up to the strain softening onset, upon which the homogenized strain stress curve looses its size objectivity in any case.

The direct finite element results of randomly picked SVEs under loading condition (65) are presented in Figs. 13(a)-13(c). It can be seen that the homogenized stress components $\sigma_{\mathrm{M} 11}$ and $\sigma_{\mathrm{M} 33}$ obtained with the MF-ROM with the effective materials properties agree well with that obtained from the direct finite element analysis, see Figs. 13(a) and 13(c); a discrepancy appears for larger strain in the trend of $\sigma_{\mathrm{M} 22}$, see Fig. 13(b). Loadingunloading cycles are studied in Fig. 13(d) and are shown to be well captured by the MF-ROM. MFH analyzes are then performed using the same identified effective properties, but by considering the loading condition (67). These results are compared to the results of direct finite element analyzes on several SVEs in Fig. 13(e). An obvious strain softening can be seen for the direct finite element results in Fig. 13(e), which is not captured by the effective matrix material properties of the MF-ROM. However, a loading-unloading cycle is well captured by the method if the unloading occurs in the first stage of the damage evolution as seen in Fig. 13(f).

One of the reason for the loss of accuracy of the stochastic MF-ROM is related to the use of a uniform isotropic damaged effective matrix to represent the real matrix. Although the damage law of the matrix phase is isotropic, the distribution in the SVE of damage during the computational homogenization is not isotropic and would require a non-isotropic damage model in the micro-mechanics-based reduced order model. This point is discussed further hereafter.

\subsubsection{The effect of damage on the accuracy of the $M F-R O M$}

The damage evolution of 2 randomly picked SVEs is presented in Fig. 14. Figure 14 shows that the damage in the matrix is almost uniform at 


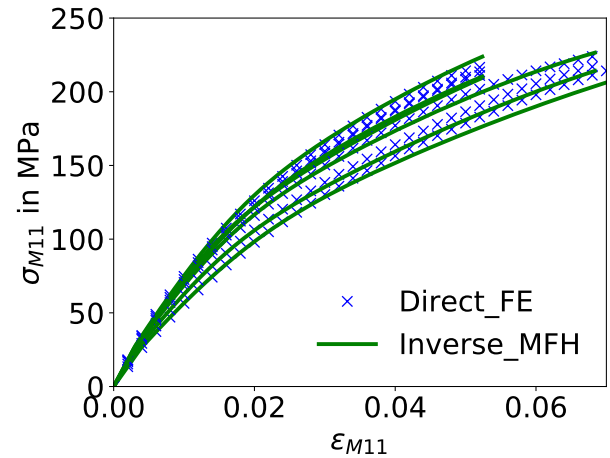

(a)

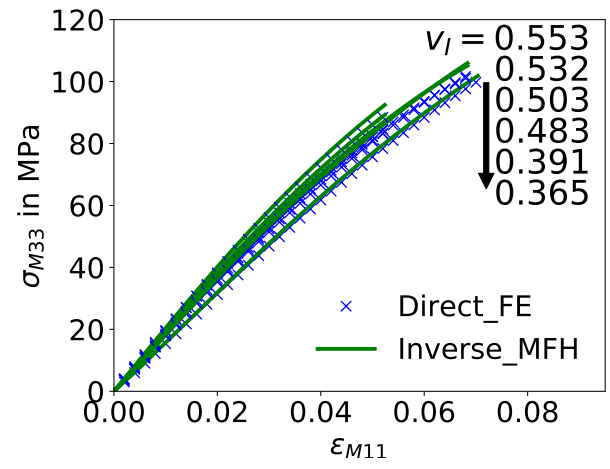

(c)

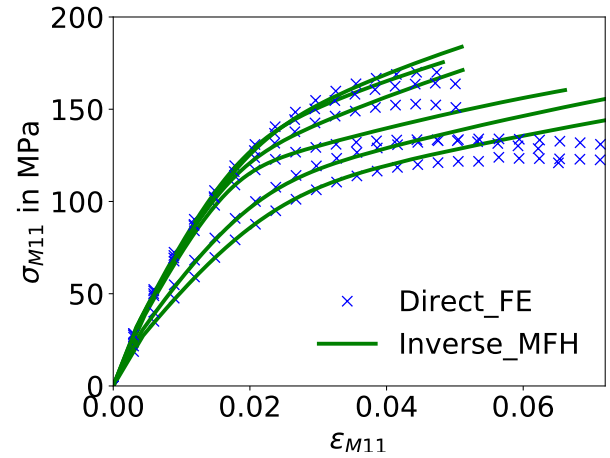

(e)

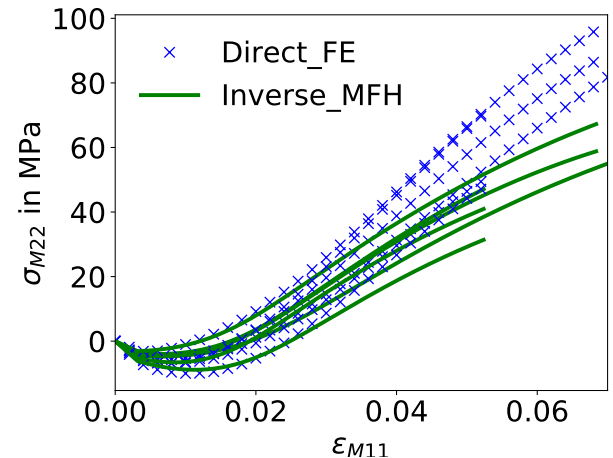

(b)

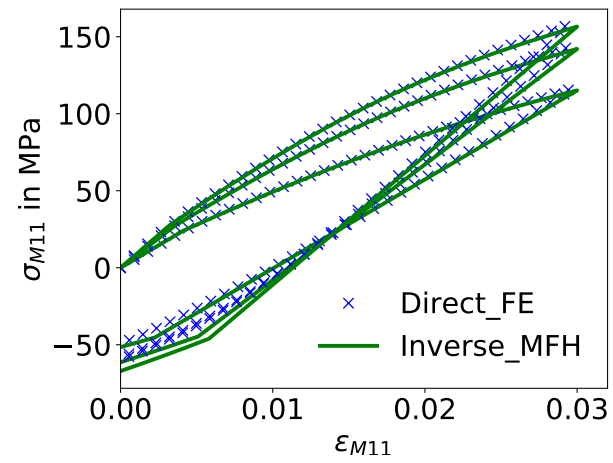

(d)

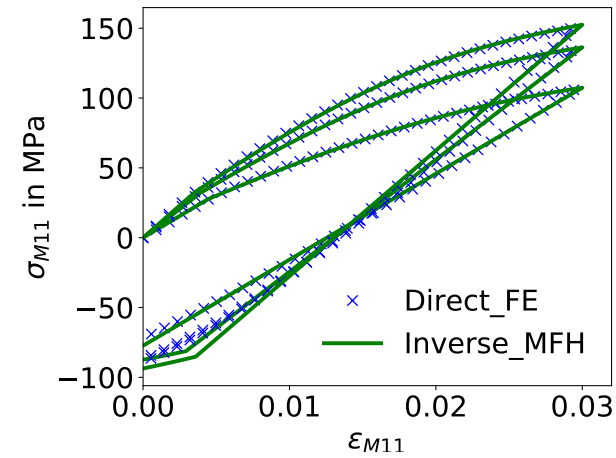

(f)

Figure 13: Homogenized response of damage-enhanced elasto-plastic SVEs: (a)-(d) Under boundary conditions (65); and (e)-(f) Under boundary condition (67).

the early stage of deformation. Therefore, the assumption of using a scalar damage variable is valid and the effective damage can be identified with the 


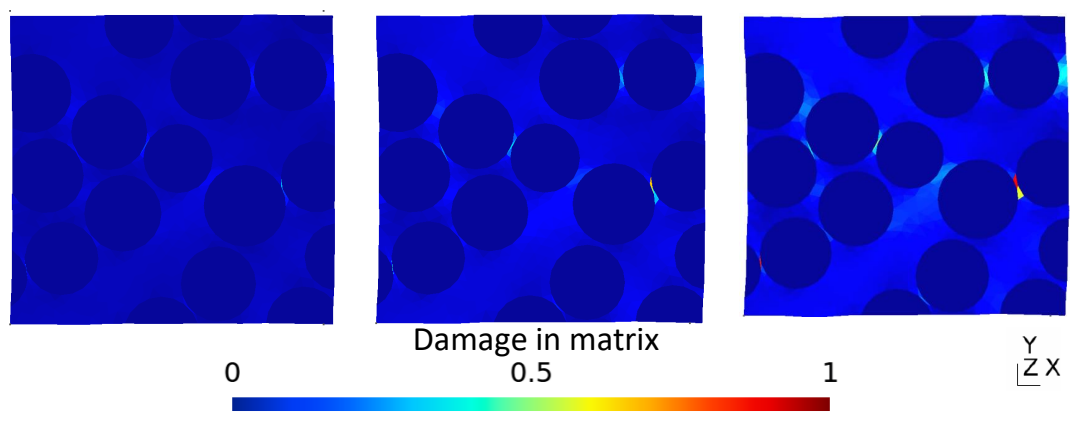

(a) SVE1
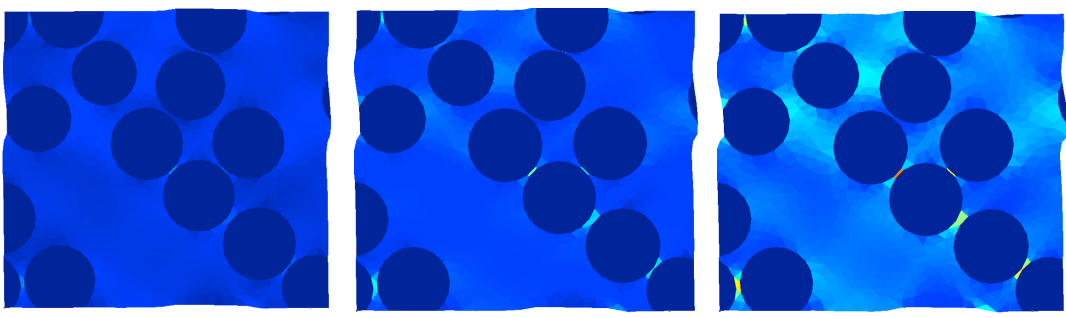

0

Damage in matrix 0.5

1

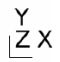

(b) SVE2

Figure 14: Damage evolution in matrix.

optimization process (58). Once the damage starts to localize at a few spots of the matrix when the deformation of the SVEs increases, since the distribution of these damage localization spots is neither uniform nor isotropic, an anisotropic behavior of the matrix appears after homogenization. The damage in the effective matrix cannot be well described with a scalar law anymore, hence, identifying the damage in the matrix with the optimization process (58) leads to unreliable results. With the MF-ROM, the maximum effective damage values $\tilde{D}_{0}$ reached in the matrix are 0.089 for SVE \#1, Fig. 14(a), and 0.187 for SVE \#2, Fig. 14(b). These effective damage values are quite close to the volume averaged damage in the matrix. However, in the direct finite simulations, the damage reaches almost 1.0 locally, and these damage localization spots have a much stronger effect on the apparent stiffness of these SVEs in the main loading direction ( $x$-direction), than in the other directions.

Because the effective damage could not be accurately extracted with the 
optimization process (58) when serious damage localization occurs, the extracted accumulated plastic strain $\tilde{p}_{0}$ and isotropic hardening stress $\tilde{R}_{0}\left(\tilde{p}_{0}\right)$ evolution, Eqs. (60) and (61), are no longer reliable, and the accuracy of of the MF-ROM decreases. Moreover, severe damage localizations normally appear together with the strain softening of SVEs, during which homogenized stress-strain relationship looses size objectivity. Another homogenization method should thus be considered beyond strain softening onset as it will be discussed in Section 6.2.

\section{Statistical analysis and generation of random material parame- ters}

In this section, in order to generate proper random fields for SFEM, some statistical features of the identified parameters of the MF-ROM are first analyzed. We consider the random effective material parameters extracted by the inverse identification process conducted in Section 4 on UD-fiber reinforced elasto-plastic matrix, successively without and with damage enhancement; the distribution of these parameters and their dependency are studied. A stochastic model is then built using the data-driven sampling method developed in [60]. It is then shown that, despite the existing dependency in the effective properties and the limited number of samples, a Markov chain Monte Carlo (MCMC) process can generate random vectors respecting these statistical features in order to conduct SFEM simulations in the next section.

\subsection{Random material parameters for UD-fiber reinforced composites}

In the presented stochastic MF-ROM, there are totally 9 and 11 random parameters being used respectively to describe the UD-fiber reinforced elastoplastic matrix without and with damage enhancement. We denote them in the form of the random vectors

- Without damage: $\boldsymbol{\beta}=\left[\tilde{\mathrm{I}}, \tilde{\theta}, v_{\mathrm{I}}, \tilde{E}_{0}, \tilde{\nu}_{0}, \tilde{\sigma}_{\mathrm{Y} 0}, \tilde{k}_{1_{0}}, \tilde{k}_{2_{0}}, \tilde{m}_{0}\right]^{\mathrm{T}}$; and

- With damage: $\boldsymbol{\beta}^{\mathrm{D}}=\left[\tilde{\mathrm{I}}, \tilde{\theta}, v_{\mathrm{I}}, \tilde{E}_{0}, \tilde{\nu}_{0}, \tilde{\sigma}_{\mathrm{Y} 0}, \tilde{k}_{1_{0}}^{\mathrm{D}}, \tilde{k}_{2_{0}}^{\mathrm{D}}, \tilde{m}_{0}^{\mathrm{D}}, \tilde{S}_{0}, \tilde{s}_{0}\right]^{\mathrm{T}}$.

According to the presented inverse MFH process, the 3 micro-structural geometrical parameters, $\tilde{\mathrm{I}}, \tilde{\theta}$ and $v_{\mathrm{I}}$, and the 3 effective matrix parameters, $\tilde{E}_{0}$, $\tilde{\nu}_{0}$ and $\tilde{\sigma}_{\mathrm{Y} 0}$ will have the same statistical features for the elasto-plastic matrix and for the damage enhanced elasto-plastic matrix. Therefore, the notations used for these six random parameters are not discriminated, contrarily to the 
hardening law parameters $\tilde{k}_{1_{0}} / \tilde{k}_{1_{0}}^{\mathrm{D}}, \tilde{k}_{2_{0}} / \tilde{k}_{2_{0}}^{\mathrm{D}}$ and $\tilde{m}_{0} / \tilde{m}_{0}^{\mathrm{D} 5}$, for the two kinds of matrix materials.

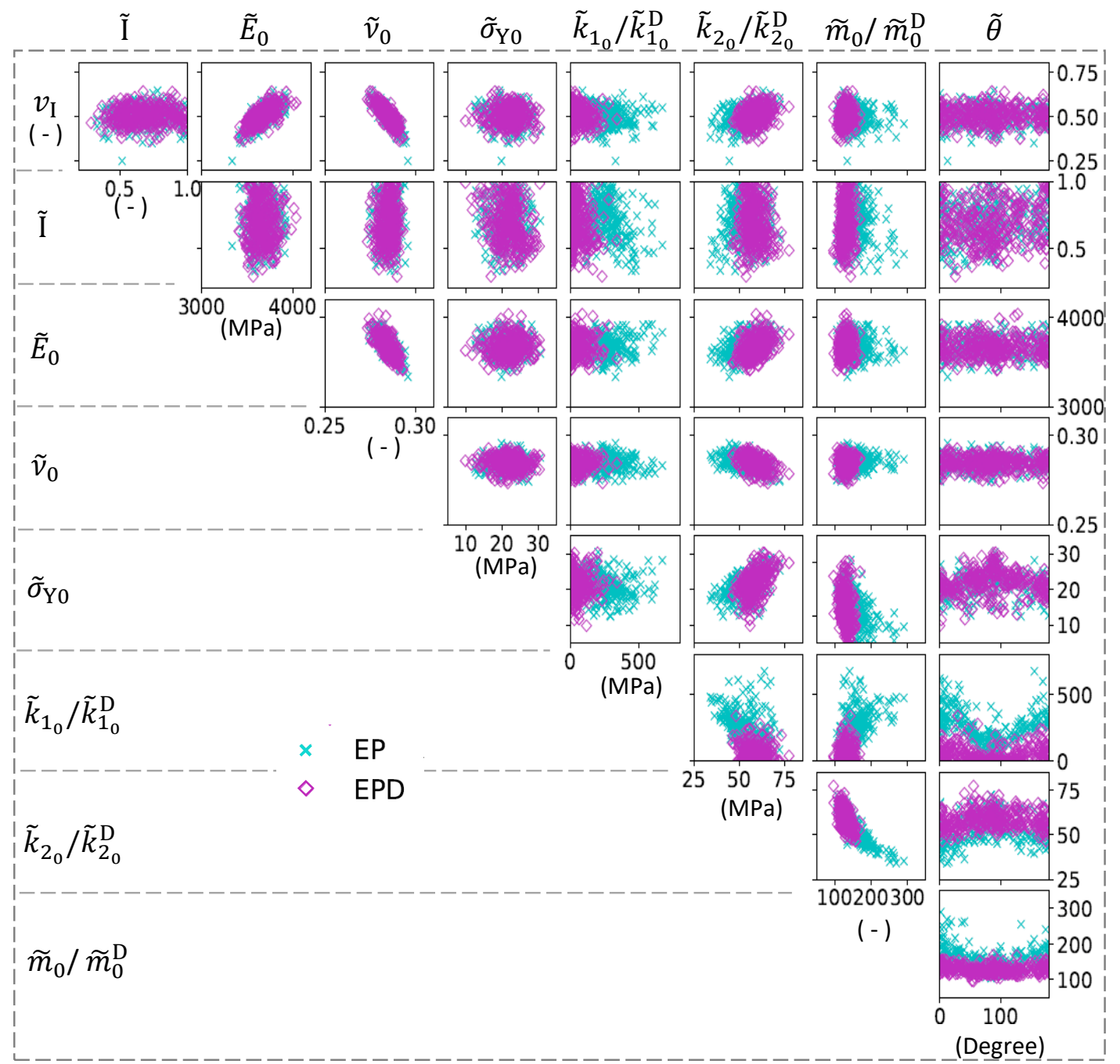

Figure 15: Randomly picked realizations of $v_{\mathrm{I}}, \tilde{\mathrm{I}}, \tilde{E}_{0}, \tilde{\nu}_{0}, \tilde{\sigma}_{Y 0}, \tilde{k}_{1_{0}} / \tilde{k}_{1_{0}}^{\mathrm{D}}, \tilde{k}_{2_{0}} / \tilde{k}_{2_{0}}^{\mathrm{D}}, \tilde{m}_{0} / \tilde{m}_{0}^{\mathrm{D}}$ and $\tilde{\theta}$. The notation $\tilde{k}_{1_{0}} / \tilde{k}_{1_{0}}^{\mathrm{D}}$ means either $\tilde{k}_{1_{0}}$ or $\tilde{k}_{1_{0}}^{\mathrm{D}}$ for respectively the elasto-plastic matrix (EP) and the damage-enhanced elasto-plastic matrix (EPD).

${ }^{5}$ The notations $\tilde{k}_{1_{0}} / \tilde{k}_{1_{0}}^{\mathrm{D}}$ means either $\tilde{k}_{1_{0}}$ or $\tilde{k}_{1_{0}}^{\mathrm{D}}$ for respectively the elasto-plastic matrix and the damage-enhanced elasto-plastic matrix. 


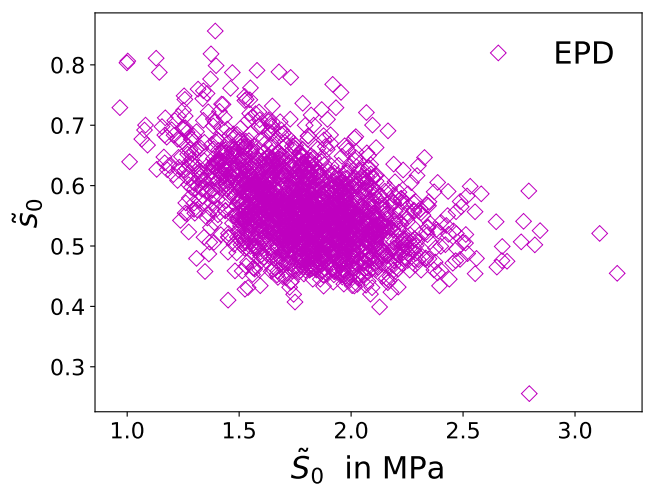

Figure 16: Randomly picked realizations of $\tilde{S}_{0}$ and $\tilde{s}_{0}$ for the damage-enhanced elastoplastic matrix.

In Fig. 15, a few randomly picked realizations of $\tilde{\mathrm{I}}, \tilde{\theta}, v_{\mathrm{I}}, \tilde{E}_{0}, \tilde{\nu}_{0}, \tilde{\sigma}_{\mathrm{Y} 0}$, $\tilde{k}_{1_{0}}, \tilde{k}_{2_{0}}, \tilde{m}_{0}$ and $\tilde{k}_{1_{0}}^{\mathrm{D}}, \tilde{k}_{2_{0}}^{\mathrm{D}}, \tilde{m}_{0}^{\mathrm{D}}$ are presented in order to study their dependency. Respecting this dependency is of prime importance in order to generate the meso-scale random properties for the stochastic finite element simulations. Only 300 realizations are used for a better visualization in both cases of elasto-plastic matrix (EP) and of damage enhanced elasto-plastic matrix (EPD). Obvious correlations can be found between the random parameters in Fig. 15, except for $\tilde{\theta}$. Since the non-local damage model is adopted in the direct finite element analysis of SVEs with damage enhanced elasto-plastic matrix, and since this non-local model introduces a volume average effect on the non-linear behavior, the identified hardening parameters are different for the two effective matrix material behaviors. In particular, the difference between $\tilde{k}_{1_{0}}$ and $\tilde{k}_{1_{0}}^{\mathrm{D}}, \tilde{k}_{2_{0}}$ and $\tilde{k}_{2_{0}}^{\mathrm{D}}$ and $\tilde{m}_{0}$ and $\tilde{m}_{0}^{\mathrm{D}}$ respectively are observed in Fig. 15.

For completeness, 1800 realizations of $\tilde{S}_{0}$ and $\tilde{s}_{0}$ are plotted in Fig. 16. The histograms of the $\tilde{\sigma}_{\mathrm{Y} 0}, \tilde{k}_{1_{0}}^{\mathrm{D}}, \tilde{k}_{2_{0}}^{\mathrm{D}}, \tilde{m}_{0}^{\mathrm{D}}, \tilde{S}_{0}$ and $\tilde{s}_{0}$ are also presented in Fig. 17, while the histograms of the $\tilde{\mathrm{I}}, \tilde{\theta}, v_{\mathrm{I}}, \tilde{E}_{0}$ and $\tilde{\nu}_{0}$ distributions are left out for conciseness since they have already been presented in [50].

In this section, we focus on the random MFH input parameters for composites with damage enhanced elasto-plastic matrix. Since the distributions of the random effective material parameters obtained from the inverse identification process are not Gaussian in general, see Fig. 17, the distance correlations are used to investigate the dependence between the 11 random parameters of the random vector $\beta^{\mathrm{D}}$. More than 1800 samples are used to 


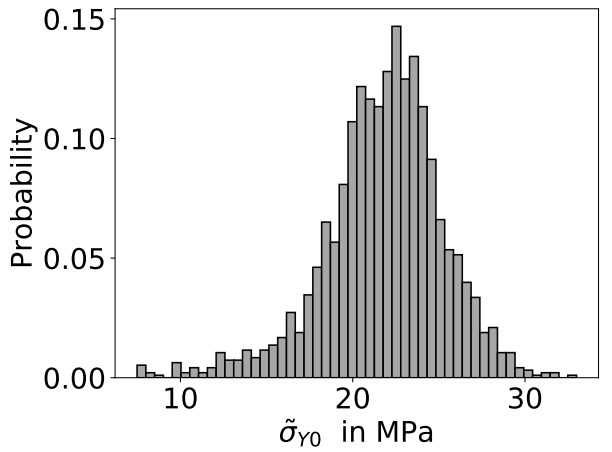

(a)

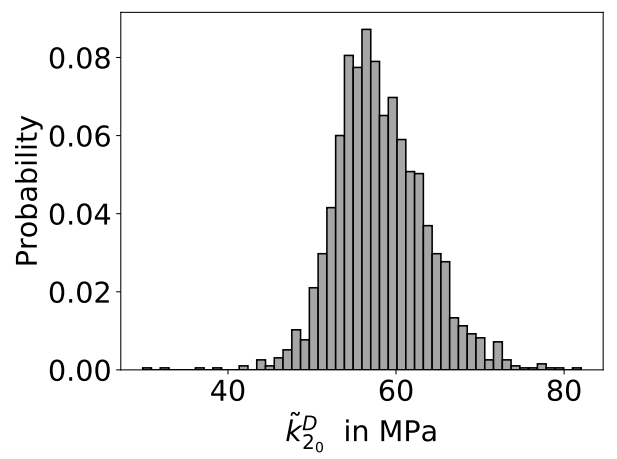

(c)

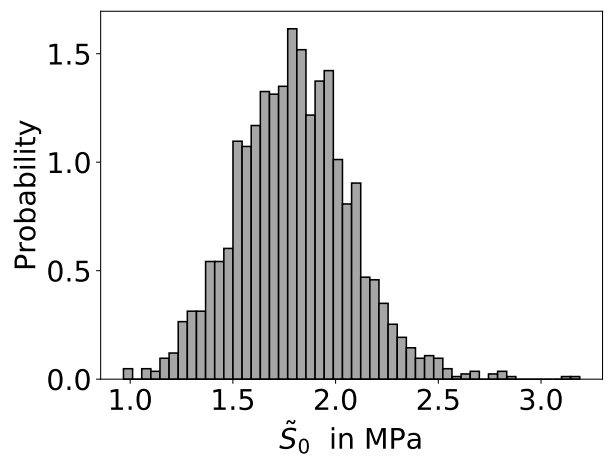

(e)

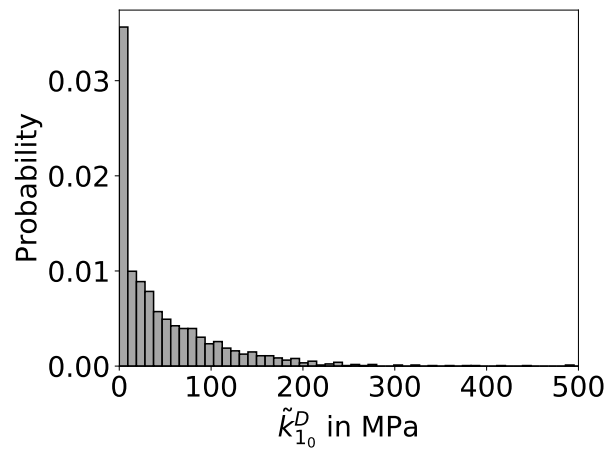

(b)

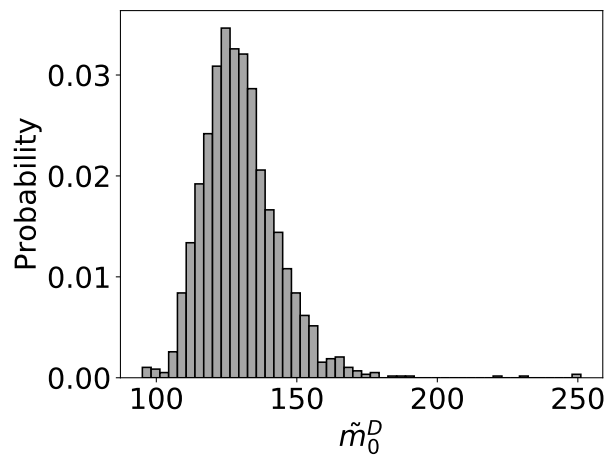

(d)

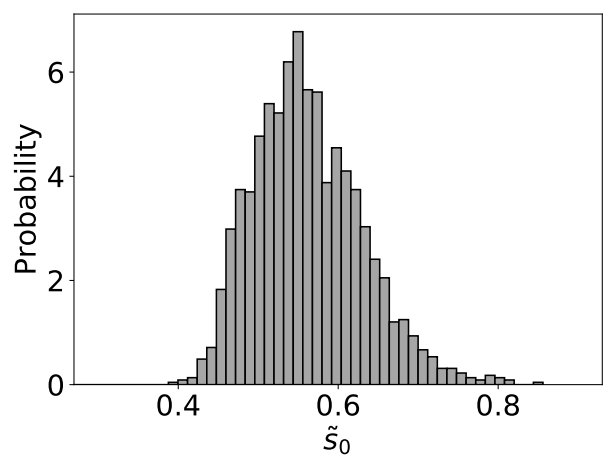

(f)

Figure 17: Histograms of (a) $\tilde{\sigma}_{\mathrm{Y} 0}$; (b) $\tilde{k}_{1_{0}}^{\mathrm{D}} ;$ (c) $\tilde{k}_{2_{0}}^{\mathrm{D}} ;$ (d) $\tilde{m}_{0}^{\mathrm{D}}$; (e) $\tilde{S}_{0}$ and (f) $\tilde{s}_{0}$ distributions for the damage-enhanced elasto-plastic matrix.

compute their distance correlations which are presented in Table 3. From Table 3 , an obvious dependence can be seen among the 11 random variables. 
Table 3: Distance correlation of effective random material parameters.

\begin{tabular}{|l|lllllllllll|}
\hline & $v_{\mathrm{I}}$ & $\tilde{\mathrm{I}}$ & $\tilde{E}_{0}$ & $\tilde{\nu}_{0}$ & $\tilde{\sigma}_{\mathrm{Y} 0}$ & $\tilde{k}_{1_{0}}^{\mathrm{D}}$ & $\tilde{k}_{2_{0}}^{\mathrm{D}}$ & $\tilde{m}_{0}^{\mathrm{D}}$ & $\tilde{\theta}$ & $\tilde{S}_{0}$ & $\tilde{s}_{0}$ \\
\hline$v_{\mathrm{I}}$ & 1.0 & 0.09 & 0.70 & 0.82 & 0.07 & 0.11 & 0.30 & 0.21 & 0.04 & 0.11 & 0.38 \\
$\tilde{\mathrm{I}}$ & & 1.0 & 0.06 & 0.06 & 0.11 & 0.10 & 0.05 & 0.08 & 0.15 & 0.18 & 0.10 \\
$\tilde{E}_{0}$ & & & 1.0 & 0.70 & 0.12 & 0.19 & 0.38 & 0.19 & 0.05 & 0.11 & 0.56 \\
$\tilde{\nu}_{0}$ & & & & 1.0 & 0.10 & 0.09 & 0.42 & 0.13 & 0.04 & 0.09 & 0.40 \\
$\tilde{\sigma}_{\mathrm{Y} 0}$ & & & & & 1.0 & 0.23 & 0.44 & 0.36 & 0.22 & 0.29 & 0.06 \\
$\tilde{k}_{1_{0}}^{\mathrm{D}}$ & & & & & & 1.0 & 0.17 & 0.16 & 0.29 & 0.37 & 0.47 \\
$\tilde{k}_{20}^{\mathrm{D}}$ & & & & & & & 1.0 & 0.51 & 0.13 & 0.17 & 0.36 \\
$\tilde{m}_{0}^{\mathrm{D}}$ & & & \multirow{2}{*}{ sym } & & & & & 1.0 & 0.16 & 0.15 & 0.08 \\
$\tilde{\theta}^{\mathrm{D}}$ & & & & & & & & & 1.0 & 0.29 & 0.25 \\
$\tilde{S}_{0}$ & & & & & & & & & & 1.0 & 0.42 \\
$\tilde{s}_{0}$ & & & & & & & & & & & 1.0 \\
\hline
\end{tabular}

\subsection{Stochastic model of the MF-ROM input parameters}

In the remaining we consider the damage-enhanced case, the elasto-plastic one being easily deduced from it. Using the realizations of random vector $\beta^{\mathrm{D}}$ as random material properties, a stochastic finite element analysis can be carried out at the higher length scale. The realizations of random vector $\beta^{\mathrm{D}}$ can be obtained directly using the presented inverse MFH process, which is computational costly and not practical. This motivates the construction of a random vector generator. Based on the available samples, empirical copula has been constructed to generate the random elastic material property vectors in [50]. However, empirical copula is not feasible in the present work. Firstly, the random vector $\beta^{\mathrm{D}}$ has a relatively high dimension of 11 , in which case constructing a reasonable empirical copula would require an enormous amount of sampling data. Secondly, the samples of $\beta^{\mathrm{D}}$ are extracted from direct non-linear elasto-plastic finite element simulations on SVEs, which is much more computational expensive compared to that of the linear elastic simulations. In order to construct a random vector generator of 11 dimensions with dependence based on a limited number of samples ( $N=1868$ samples), a sophisticated data-driven sampling method [60] is adopted in this work. 


\subsubsection{Data-driven probability sampling}

The data-driven sampling method, which has been presented in reference [60], is summarized in this section.

Considering the damage-enhanced case, the random material properties are represented by a random vector $\beta^{\mathrm{D}}$, which is defined on a probability space $(\Theta, \mathcal{T}, \mathcal{P})$, with value in $\mathbb{R}^{n}(n=11$ in our case). The distribution of this random vector is unknown, while, it is assumed that its statistic information is concentrated on a subset $\mathcal{S}^{n}$ of $\mathbb{R}^{n}$. The available information consists of a given set of $N$ statistically independent realizations $\beta^{\mathrm{D}}(\theta)$, here the realizations illustrated in Section 5.1, where $\theta \in \Theta$. It is further assumed that the local structure of the given data-set is preserved via a random matrix $\left[\beta^{\mathrm{D}}\right]$, which is defined on $(\Theta, \mathcal{T}, \mathcal{P})$, with value in $\mathbb{M}_{n, N}$. Specifically, $\left[\beta^{\mathrm{D}}\right]=$ $\left[\beta_{1}^{\mathrm{D}}, \ldots, \beta_{N}^{\mathrm{D}}\right]$, with each column $\beta_{i}^{\mathrm{D}}(i=1, \ldots, N)$ being an independent copy of the random vector $\beta^{\mathrm{D}}$. Therefore, if the vector $\mathrm{b}$ denotes a realization of $\beta^{\mathrm{D}}$, the matrix $[\mathbf{b}]=\left[\mathbf{b}_{1}, \ldots, \mathbf{b}_{N}\right]$ is a realization of $\left[\beta^{\mathrm{D}}\right]$ (with $N=1868$ observation samples in our case). A MCMC process is then used to generate extra data samples based on the matrix $[\mathbf{b}]$.

This random samples generating process can be carried out in three sequential sub-processes:

1. Preparation of data-set by removing the mean and scaling it to the unit variance;

2. Random data generating process; and

3. Recovering generated data in the original data-set scale.

These three steps are summarized in Appendix D.

Finally, $k \times N$ additional realizations of $\beta^{D}$ can be generated, with $k=$ $1, \ldots, n_{\mathrm{MC}}$, where $n_{\mathrm{MC}}$ corresponds to the maximum number of required additional realizations, see details in Appendix D.

\subsubsection{Application of the data-driven sampling method}

In this section, the data-driven sampling method summarized in Section 5.2.1 is applied using the $N=1868$ realizations of the random vector $\beta^{\mathrm{D}}$, which were obtained from the inverse $\mathrm{MFH}$ analysis of direct finite element simulations on SVEs in Section 5.1.

With $n_{\mathrm{MC}}=10,18680$ additional realizations of $\beta^{\mathrm{D}}$ are generated. 300 samples are randomly drew from the 18680 generated realizations and 300 other samples from the 1868 given realizations obtained from the inverse 


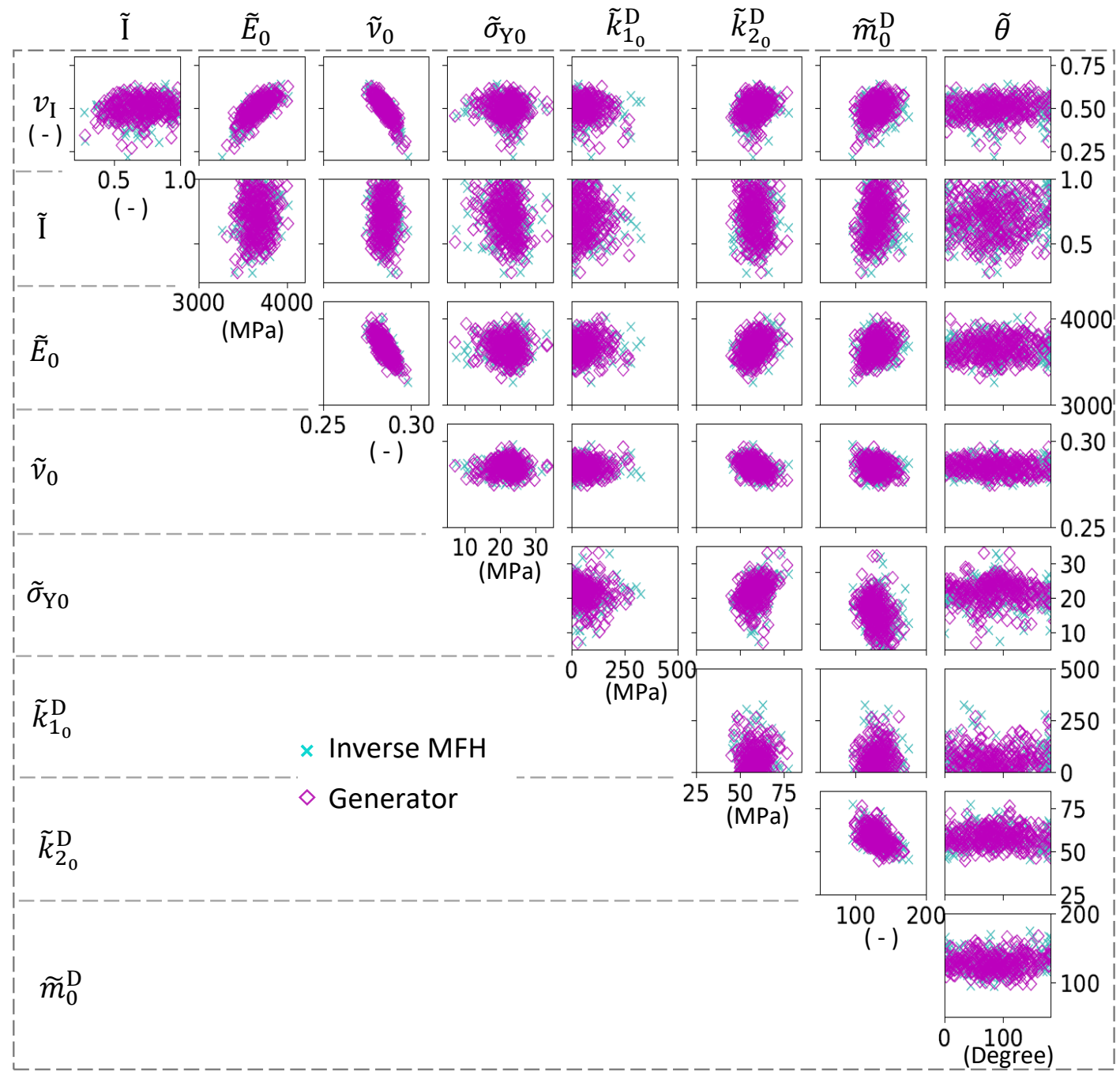

Figure 18: Comparison of the generated samples and the original observations for $\tilde{\mathrm{I}}, \tilde{\theta}, v_{\mathrm{I}}$, $\tilde{E}_{0}, \tilde{\nu}_{0}, \tilde{\sigma}_{\mathrm{Y} 0}, \tilde{k}_{1_{0}}^{\mathrm{D}}, \tilde{k}_{2_{0}}^{\mathrm{D}}$ and $\tilde{m}_{0}^{\mathrm{D}}$.

MFH analysis. They are reported in Figs. 18 and 19 for the purpose of comparison. Very good agreements between the distributions of the generated data and the original observation data can be seen. 


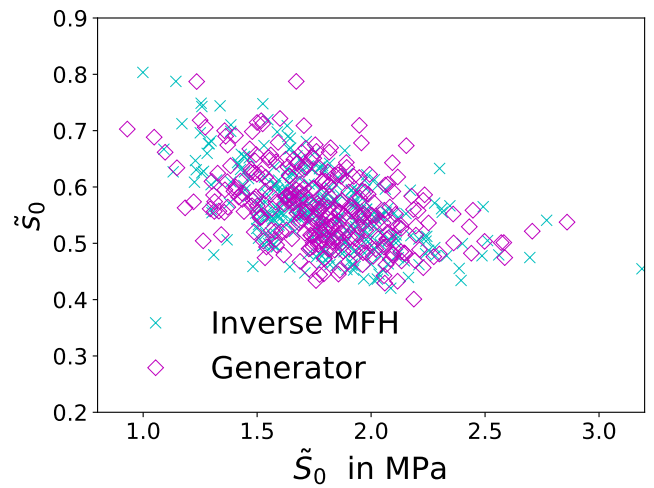

Figure 19: Comparison of the generated samples and the original observations for $\tilde{S}_{0}$ and $\tilde{s}$.

\section{Stochastic analysis of a transverse tensile test on a ply}

In this section, in order to illustrate the applicability of the stochastic MFROM, we study the response of a UD composite ply loaded in the transverse direction. First the description of the test, in particular the discretization of the stochastic field and of the finite elements, is detailed before applying the stochastic MF-ROM enhanced by the non-local damage formulation. A few realizations of the tensile tests are then studied to illustrate the stochastic effect on a strain-stress curve. For each realization, a random field is generated by the stochastic model described in Section 5.2 and serves as input for MF-ROM.

\subsection{Tensile test setup}

A composite cross-section of $500 \times 250 \mu \mathrm{m}^{2}$ is loaded in the transverse $x$-direction, with plane-strain condition along the longitudinal $z$-direction of fibers, and surface-traction-free condition along the $y$-direction. Its finite element discretization along with the applied boundary and loading conditions is presented in Fig. 20.

The case of the damage enhanced elasto-plastic matrix is considered in this test. The random field of the material properties is discretized into squares of $25 \times 25 \mu \mathrm{m}^{2}$, and the random material properties of each square are independent from the properties of the neighboring squares since the spatial correlation vanishes as shown in [14]. The applied material properties are realizations of the random vector $\beta^{\mathrm{D}}$, which are generated values through the process described in Section 5.2. The discretization of the random material 


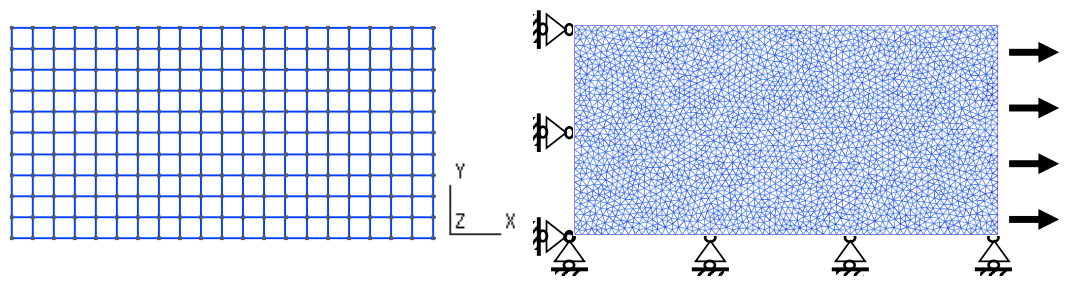

Figure 20: Tensile stochastic finite element analysis: discretizations of the random field (left) and of the finite element structure (right).

properties field is also shown in Fig. 20. In order to avoid a strong contrast of material properties, which may lead to an artificial stress concentration, smooth-step functions are used to describe the transition of the material properties at the internal boundaries of the random field discretization [14].

The non-local formulation of damage is also used at the ply level in order to avoid mesh dependency effects. At the ply level, the non-local equations read

$$
\left\{\begin{array}{l}
\nabla_{\mathrm{M}} \cdot \boldsymbol{\sigma}_{\mathrm{M}}=0 \quad \forall \boldsymbol{x} \in \Omega, \\
\underline{p}-\nabla \cdot \boldsymbol{c}_{\mathrm{g}} \cdot \nabla \underline{p}=p_{0} \quad \forall \boldsymbol{x} \in \Omega, \\
\boldsymbol{n}_{\mathrm{M}} \cdot \boldsymbol{\sigma}_{\mathrm{M}}=\boldsymbol{t}_{\mathrm{M}} \quad \forall \boldsymbol{x} \in \partial \Omega, \\
\boldsymbol{n}_{\mathrm{M}} \cdot \boldsymbol{c}_{\mathrm{g}} \cdot \nabla \underline{p}=0 \quad \forall \boldsymbol{x} \in \partial \Omega,
\end{array}\right.
$$

where $\boldsymbol{\sigma}_{\mathrm{M}}$ results from the MF-ROM following the resolution process described in Appendix $\mathrm{C}$ and where the anisotropic form of $\boldsymbol{c}_{\mathrm{g}}=\operatorname{diag}\left(l_{x}^{2}, l_{y}^{2}, l_{z}^{2}\right)$ is used. In this case, we consider $l_{x}=l_{y}=\frac{25}{\sqrt{2}} \mu \mathrm{m}$ according to the size of SVEs, $25 \times 25 \mu \mathrm{m}^{2}$, and we keep $l_{z}=\sqrt{2} \mathrm{~mm}$, since the interaction along the fiber direction ranges on a longer distance.

Figures 21(a) and 21(b) show respectively the homogenized transverse stress of the composite material, and the accumulated plastic strain of the matrix at the ply loading strain $\varepsilon_{\mathrm{M}_{11}}=0.08$. These fields are quite even in each $25 \times 25 \mu \mathrm{m}^{2}$ random field discretization cell. Locally, the damage start to evolve in the matrix at different strain states of composites and the different damage evolution rates can be observed by comparing Fig. 21(c) and Fig. $21(\mathrm{~d})$, in which the spots of the highest damage at the average loading strain $\varepsilon_{\mathrm{M}_{11}}=0.04$ changed as the macro-strain increases to $\varepsilon_{\mathrm{M}_{11}}=0.08$.

The obvious effect of random material properties can be seen from Figs. 21(a) and 21(b), in which the location where a higher homogenized transverse stress is reached in the composite material does not necessarily correspond 


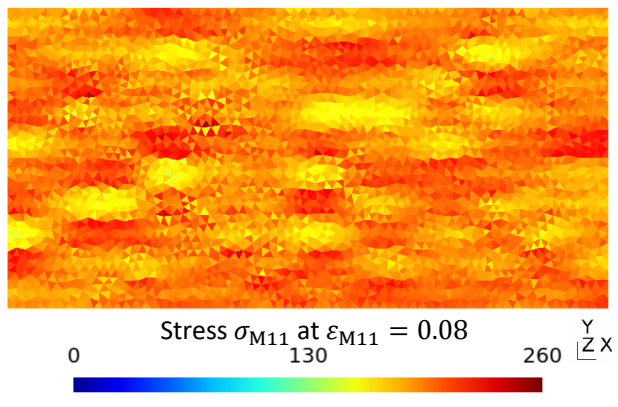

(a)

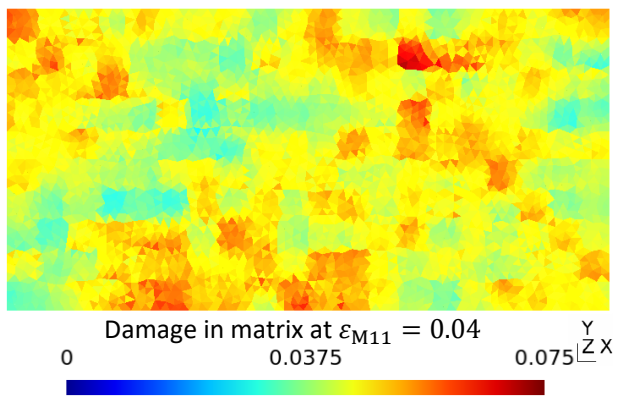

(c)

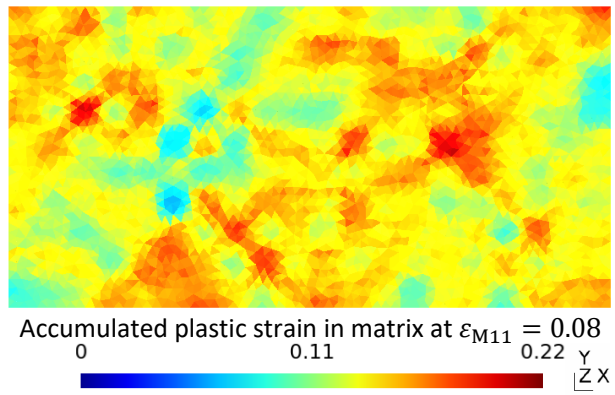

(b)

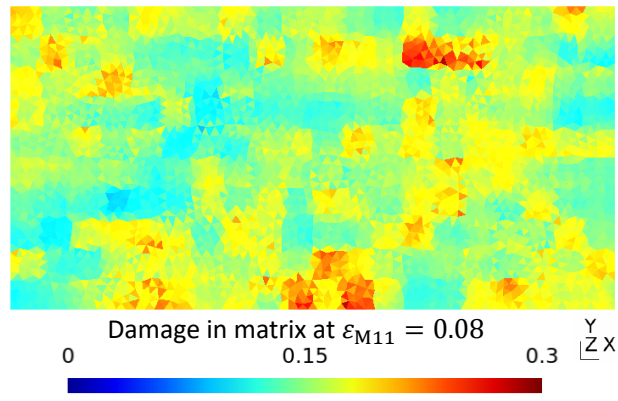

(d)

Figure 21: The distributions of macro-scale stress of the composite material, accumulated plastic strain and damage of effective matrix in the $500 \times 250 \mu \mathrm{m}^{2}$ cross-section under transverse loading.

to the location where a higher accumulated plastic strain is obtained in the matrix phase; indeed, the location associated to a lower homogenized stress associates with a SVE of low fiber volume fraction, for which the plastic strain in the matrix phase starts to develop sooner. According to the applied damage model in this work, the earlier development of plasticity in the matrix leads to an earlier evolution of its damage. Therefore, the location corresponding to a high accumulated plastic strain in the matrix phase may associate with a high damage value. However, it is only the case at the early stage of loading as it can be seen by comparing Figs. 21(c) and 21(d). Indeed, for the material points with effective material properties associated to SVEs of high fiber volume fraction, the plastic strain and damage in the matrix initiate at a higher stress state. In that case, although the level of plastic strain is not high, the effective damage evolution in the matrix can be faster, see Fig, 21(d), because of the localization effect in the micro-structure. 


\subsection{Study of different ply realizations}

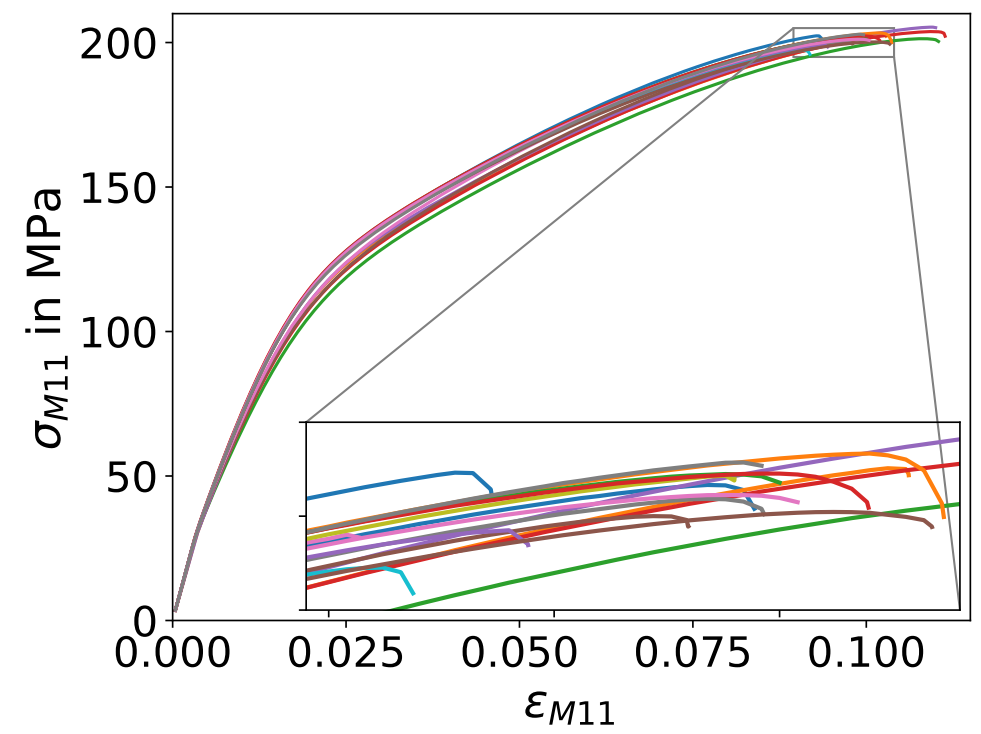

Figure 22: The strain-stress curves of five $500 \times 250 \mu \mathrm{m}^{2}$ tensile test samples.

The analysis conducted in the previous paragraph is now repeated for 18 ply realizations. The resulting strain-stress curves are presented in Fig. 22 up to the point of failure. We however note that the developed multiscale approach looses objectivity when the homogenized stress-strain relationship reaches strain softening onset. Beyond that point, the homogenized behavior should be formulated in terms of other quantities for which size objectivity can be recovered, such as fracture toughness or traction-displacement jump softening response [89, 90, 91, 92]. With a view to the development of a stochastic multiscale process, which can be used to carry out a complete structural analysis including the degradation and failure of composite structures, the localization stages should thus be up-scaled using other indicator such as fracture toughness, which also exhibits uncertainties [71, 93]. During structural analyzes, damage localization zones can be substituted by a crack at the onset of strain softening of local material [94, 95] and the corresponding cohesive laws can be extracted from the simulations of SVEs $[92,71]$. An alternative is to extract parameters of a phase-field model [93] from lower scale simulations. The maximum loading in the curves presented in Fig. 22 should thus be seen as a trend study and not as a rigorous failure analysis: the presented inverse MFH analysis cannot reflect the effect of damage local- 
ization in SVEs, and the MFH analysis with the effective material properties usually postpones the failure of SVEs, see Fig. 13. Therefore, the failure points in Fig. 22 are postponed results comparing to the reality.

When analyzing the stress-strain curves of Fig. 22, it appears that, during the first stage of the loading, the plies do not exhibit a difference in their stiffness. This results from the adopted SVE micro-structure generator, which is based on the assumption that the statistical descriptors of fiber distribution are homogeneous or stationary in space [14]. With the increase of testing sample's size, more and more effective properties of SVEs are used in the analysis, leading to a convergence of the stiffness to a unique value. However, there exists a discrepancy in the ply responses in the non-linear stage and in the composite material strength.

\section{Conclusions}

In this work, a MF-ROM, which was developed for linear elastic composites in [50], is extended to the non-linear regime. The main purpose of the presented inverse $\mathrm{MFH}$ procedure is to improve the computational efficiency of stochastic multiscale analyzes while relying on micro mechanics models. Using the incremental-secant MFH formulation as a core, the uncertainty observed in the response obtained by direct finite element simulations of composite SVEs, and which results from the uncertainties of their micro-structures, is represented by random effective matrix properties and geometrical parameters. Compared to the direct finite element analyzes on SVEs, the MF-ROM reduces not only the computational cost, but also the order of uncertain parameters in the composite micro-structures.

The accuracy of the presented procedure is evaluated by comparing the results of direct finite element simulations on SVE realizations with the homogenized behaviors predicted by the MF-ROM for the input parameters observations extracted using the developed inverse identification process. The observed relative error in the loading direction is within $3.5 \%$ for the composites made of an elasto-plastic matrix, and within $6 \%$, before the softening stage, for the composites made of a damage-enhanced elasto-plastic matrix. When considering the stress components transverse to the loading direction or different loading conditions, the error is more important because the considered composite material is characterized, on the one hand, by a high volume fraction of inclusions, and, on the other hand, by a ductile matrix phase, which correspond to a severe situation. 
A stochastic model is then built using a data-driven sampling process in order to be able to generate random vectors as input for the MF-ROM used in the SFEM. In order to illustrate the potential of the methodology, the strength under transverse loading is extracted for several composite ply realizations. However, in this proposed work, although a damage model is considered at both scales, we did not account for the loss of objectivity in the scale transition during local softening. In a future work, the damage to crack transition at the onset of strain softening will also be studied in a stochastic way to reduce the error caused by damage localization.

\section{Appendix A. Tensorial operations and notations}

- Dots and colons are used to indicate tensor products contracted over one and two indices respectively:

$$
\begin{aligned}
\boldsymbol{u} \cdot \boldsymbol{v}=u_{i} v_{i}, & (\boldsymbol{a} \cdot \boldsymbol{u})_{i}=a_{i j} u_{j} \\
(\boldsymbol{a} \cdot \boldsymbol{b})_{i j}=a_{i k} b_{k j}, & \boldsymbol{a}: \boldsymbol{b}=a_{i j} b_{j i} ; \\
(\mathbb{C}: \boldsymbol{a})_{i j}=\mathbb{C}_{i j k l} a_{l k}, & (\mathbb{C}: \mathbb{D})_{i j k l}=\mathbb{C}_{i j m n} \mathbb{D}_{n m k l} .
\end{aligned}
$$

- Dyadic products are designated by $\otimes$ :

$$
(\boldsymbol{u} \otimes \boldsymbol{v})_{i j}=u_{i} v_{j}, \quad(\boldsymbol{a} \otimes \boldsymbol{b})_{i j k l}=a_{i j} b_{k l} .
$$

- Symbols 1 and $\mathbb{I}$ designate the second- and fourth-order symmetric identity tensors respectively:

$$
\mathbf{1}_{i j}=\delta_{i j}, \quad \mathbb{I}_{i j k l}=\frac{1}{2}\left(\delta_{i k} \delta_{j l}+\delta_{i l} \delta_{j k}\right),
$$

where $\delta_{i j}=1$ if $i=j, \delta_{i j}=0$ if $i \neq j$.

- The spherical and deviatoric operators are $\mathbb{I}^{\text {vol }}$ and $\mathbb{I}^{\text {dev }}$ respectively:

$$
\mathbb{I}^{\mathrm{vol}} \equiv \frac{1}{3} \mathbf{1} \otimes \mathbf{1}, \quad \mathbb{I}^{\mathrm{dev}}=\mathbb{I}-\mathbb{I}^{\mathrm{vol}}
$$

so that for symmetric tensors $a_{i j}=a_{j i}$ we have:

$$
\mathbb{I}^{\mathrm{vol}}: \boldsymbol{a}=\frac{1}{3} a_{m m} \mathbf{1}, \quad \mathbb{I}^{\mathrm{dev}}: \boldsymbol{a}=\boldsymbol{a}-\frac{1}{3} a_{m m} \mathbf{1}=\operatorname{dev}(\boldsymbol{a}) .
$$




\section{Appendix B. The Lemaitre-Chaboche ductile damage model in non-local form}

In the "time-continuum" formulation of Section 2.1.2, a "continuum" elasto-plastic tangent operator such that $\dot{\hat{\boldsymbol{\sigma}}}=\mathbb{C}^{\mathrm{ep}}: \dot{\boldsymbol{\varepsilon}}$ can be calculated from (e.g. [96, chapter 12]):

$$
\mathbb{C}^{\mathrm{ep}}=\mathbb{C}^{\mathrm{el}}-\frac{(2 \mu)^{2}}{h} \boldsymbol{N} \otimes \boldsymbol{N}, \quad h=3 \mu+\frac{\mathrm{d} R}{\mathrm{~d} p}>0,
$$

where $\mu$ is the shear modulus. Due to the finite increments of strain and stress between time $t_{n}$ and time $t_{n+1}$, the material operator is actually the

algorithmic one $\mathbb{C}^{\text {alg }}=\frac{\partial \Delta \hat{\sigma}}{\partial \Delta \varepsilon}$ and differs from $\mathbb{C}^{\mathrm{ep}}$, [97]. In the case of the radial return mapping assumption, the derivative of the undamaged stress increment with respect to the strain increment reads (e.g. [96, chapter 12])

$$
\mathbb{C}^{\mathrm{alg}}=\mathbb{C}^{\mathrm{ep}}-(2 \mu)^{2}(\Delta p) \frac{\hat{\sigma}^{\mathrm{eq}}}{\hat{\sigma}^{\mathrm{eq}, \mathrm{tr}}} \frac{\partial \boldsymbol{N}}{\partial \hat{\boldsymbol{\sigma}}} \text {, with } \frac{\partial \boldsymbol{N}}{\partial \hat{\boldsymbol{\sigma}}}=\frac{1}{\hat{\sigma}^{\mathrm{eq}}}\left(\frac{3}{2} \mathbb{I}^{\mathrm{dev}}-\boldsymbol{N} \otimes \boldsymbol{N}\right) \text {. }
$$

In this last relation, $\hat{\sigma}^{\mathrm{eq}, \mathrm{tr}}$ is the trial (elastic predictor) value of $\hat{\sigma}^{\mathrm{eq}}$, and $\Delta p$ is the accumulated plastic strain increment in the time interval. Note that in case of plastic flow both $\mathbb{C}^{\mathrm{ep}}$ and $\mathbb{C}^{\text {alg }}$ are anisotropic, and that in case of elasticity $\mathbb{C}^{\text {alg }}$ reduces to $\mathbb{C}^{\mathrm{el}}$.

The material operators can then be obtained. The ones related to the Cauchy stress tensor are directly obtained following

$$
\begin{aligned}
\mathbb{C} & =\frac{\partial \Delta \boldsymbol{\sigma}}{\partial \Delta \boldsymbol{\varepsilon}}=(1-D) \mathbb{C}^{\text {alg }}-\hat{\boldsymbol{\sigma}} \otimes \frac{\partial D}{\partial \boldsymbol{\varepsilon}}, \\
\boldsymbol{C}^{u \underline{p}} & =\frac{\partial \Delta \boldsymbol{\sigma}}{\partial \underline{p}}=-\hat{\boldsymbol{\sigma}} \frac{\partial D}{\partial \underline{p}} .
\end{aligned}
$$

and the material operators related to the equivalent plastic strain following

$$
\begin{aligned}
\boldsymbol{C}^{p u} & =\frac{\partial p}{\partial \Delta \varepsilon}=\frac{2 \mu}{h} \boldsymbol{N}, \\
C^{p \underline{p}} & =\frac{\partial p}{\partial \underline{p}}=0 .
\end{aligned}
$$

These expressions are completed by the linearization of the damage law (68) written in the incremental form following [88]:

$$
\Delta D=\left(\frac{Y_{n+\alpha}}{S_{0}}\right)^{s} \Delta \underline{p},
$$


where

$$
Y=\frac{1}{2} \varepsilon^{\mathrm{e}}: \mathbb{C}^{\mathrm{el}}: \varepsilon^{\mathrm{e}} \quad \text { and } \quad Y_{n+\alpha}=(1-\alpha) Y_{n}+\alpha Y_{n+1}
$$

It can be easily deduced that

$$
\frac{\partial Y}{\partial \varepsilon^{\mathrm{e}}}: \frac{\partial \varepsilon^{\mathrm{e}}}{\partial \varepsilon}: \delta \varepsilon=\alpha \varepsilon^{\mathrm{e}}: \mathbb{C}^{\mathrm{alg}}: \delta \varepsilon,
$$

leading to

$$
\begin{aligned}
\delta D(\varepsilon, \underline{p}) & \approx \frac{\partial \Delta D}{\partial Y} \frac{\partial Y}{\partial \varepsilon^{\mathrm{e}}}: \frac{\partial \varepsilon^{\mathrm{e}}}{\partial \varepsilon}: \delta \varepsilon+\frac{\partial \Delta D}{\partial \underline{p}} \delta \underline{p} \\
& =s \Delta \underline{p} \frac{\left(Y_{n+\alpha}\right)^{s-1}}{S_{0}^{s}} \frac{\partial Y}{\partial \varepsilon^{\mathrm{e}}}: \frac{\partial \varepsilon^{\mathrm{e}}}{\partial \boldsymbol{\varepsilon}}: \delta \varepsilon+\left(\frac{Y_{n+\alpha}}{S_{0}}\right)^{s} \delta \underline{p} \\
& =\alpha s \Delta \underline{p} \frac{\left(Y_{n+\alpha}\right)^{s-1}}{S_{0}^{s}} \varepsilon^{\mathrm{e}}: \mathbb{C}^{\mathrm{alg}}: \delta \varepsilon+\left(\frac{Y_{n+\alpha}}{S_{0}}\right)^{s} \delta \underline{p}
\end{aligned}
$$

\section{Appendix C. MFH-homogenization based multiscale method}

In this section we assume that the matrix phase $\omega_{0}$ follows a damageenhanced material law and that the inclusions phase $\omega_{\text {I }}$ follows a damage-free elasto-plastic model. In that case, only a non-local internal variable $\underline{p}$ related to the matrix phase has to be considered.

The set of equations (38) was rewritten [80] as

$$
0=\boldsymbol{F}=\mathbb{C}_{0}^{\mathrm{SD}}:\left[\langle\Delta \varepsilon\rangle_{\mathrm{I}}^{\mathrm{r}}-\frac{1}{v_{0}} \mathbb{S}^{-1}:\left(\langle\Delta \varepsilon\rangle_{\mathrm{I}}^{\mathrm{r}}-\Delta \varepsilon_{\mathrm{M}}^{\mathrm{r}}\right)\right]-\mathbb{C}_{\mathrm{I}}^{\mathrm{S}}:\langle\Delta \varepsilon\rangle_{\mathrm{I}}^{\mathrm{r}},(\mathrm{C}
$$

which can be linearized as

$$
\mathrm{d} \boldsymbol{F}=\frac{\partial \boldsymbol{F}}{\partial\langle\boldsymbol{\varepsilon}\rangle_{\mathrm{I}}}: \mathrm{d}\langle\Delta \boldsymbol{\varepsilon}\rangle_{\mathrm{I}}^{\mathrm{r}}+\frac{\partial \boldsymbol{F}}{\partial\langle\boldsymbol{\varepsilon}\rangle_{0}}: \mathrm{d}\langle\Delta \boldsymbol{\varepsilon}\rangle_{0}^{\mathrm{r}}+\frac{\partial \boldsymbol{F}}{\Delta \boldsymbol{\varepsilon}_{\mathrm{M}}^{\mathrm{r}}}: \mathrm{d} \Delta \boldsymbol{\varepsilon}_{\mathrm{M}}^{\mathrm{r}}+\frac{\partial \boldsymbol{F}}{\partial \underline{p}} \mathrm{~d} \underline{p}(\mathrm{C} .2)
$$

When solving $\boldsymbol{F}=0$ at constant $\Delta \boldsymbol{\varepsilon}_{\mathrm{M}}^{\mathrm{r}}$ and constant $\underline{p}$, since $v_{0}\langle\Delta \boldsymbol{\varepsilon}\rangle_{0}^{\mathrm{r}}+$ $v_{\mathrm{I}}\langle\Delta \varepsilon\rangle_{\mathrm{I}}^{\mathrm{r}}$ is constant, Eq. (C.1) is solved iteratively with $\mathrm{d} \boldsymbol{F}=\mathbb{J}: \mathrm{d}\langle\Delta \varepsilon\rangle_{\mathrm{I}}^{\mathrm{r}}$ 
with $\mathbb{J}=\frac{\partial \boldsymbol{F}}{\partial\langle\Delta \boldsymbol{\varepsilon}\rangle_{\mathrm{I}}^{\mathrm{r}}}+\frac{\partial \boldsymbol{F}}{\partial\langle\Delta \boldsymbol{\varepsilon}\rangle_{0}^{\mathrm{r}}}: \frac{\partial\langle\Delta \boldsymbol{\varepsilon}\rangle_{0}^{\mathrm{r}}}{\partial\langle\Delta \boldsymbol{\varepsilon}\rangle_{\mathrm{I}}^{\mathrm{r}}}$ given by

$$
\begin{aligned}
\mathbb{J}= & \mathbb{C}_{0}^{\mathrm{SD}}:\left[\mathbb{I}-\mathbb{S}^{-1}\right]-\mathbb{C}_{\mathrm{I}}^{\mathrm{S}}-\frac{\partial \mathbb{C}_{\mathrm{I}}^{\mathrm{S}}}{\partial\langle\Delta \varepsilon\rangle_{\mathrm{I}}^{\mathrm{r}}}:\langle\Delta \varepsilon\rangle_{\mathrm{I}}^{\mathrm{r}}- \\
& \frac{v_{\mathrm{I}}}{v_{0}}\left(\frac{\partial \mathbb{C}_{0}^{\mathrm{SD}}}{\partial\langle\Delta \varepsilon\rangle_{0}^{\mathrm{r}}}+\frac{\partial \mathbb{C}_{0}^{\mathrm{SD}}}{\partial D_{0}} \frac{\partial D_{0}}{\partial\langle\Delta \varepsilon\rangle_{0}^{\mathrm{r}}}\right):\left[\langle\Delta \varepsilon\rangle_{\mathrm{I}}^{\mathrm{r}}-\mathbb{S}^{-1}: \frac{\left(\langle\Delta \varepsilon\rangle_{\mathrm{I}}^{\mathrm{r}}-\Delta \varepsilon_{\mathrm{M}}^{\mathrm{r}}\right)}{v_{0}}\right]- \\
& \frac{v_{\mathrm{I}}}{v_{0}^{2}} \mathbb{C}_{0}^{\mathrm{SD}} \otimes\left(\langle\Delta \varepsilon\rangle_{\mathrm{I}}^{\mathrm{r}}-\Delta \varepsilon_{\mathrm{M}}^{\mathrm{r}}\right)::\left(\mathbb{S}^{-1} \otimes \mathbb{S}^{-1}\right)::\left(\frac{\partial \mathbb{S}}{\partial\langle\varepsilon\rangle_{0}}+\frac{\partial \mathbb{S}}{\partial D_{0}} \frac{\partial D_{0}}{\partial\langle\varepsilon\rangle_{0}}\right)- \\
& \frac{v_{\mathrm{I}}}{v_{0}} \mathbb{C}_{0}^{\mathrm{SD}}: \mathbb{S}^{-1} .
\end{aligned}
$$

The derivatives of the incremental-secant operators (42) read [80]

$$
\left\{\begin{array}{l}
\frac{\partial \mathbb{C}^{\mathrm{S}}}{\partial\langle\Delta \boldsymbol{\varepsilon}\rangle^{\mathrm{r}}}=2 \mathbb{I}^{\operatorname{dev}} \otimes\left[\frac{1}{6 \mu^{\mathrm{S}}\left(\left(\langle\Delta \varepsilon\rangle^{\mathrm{r}}\right)^{\mathrm{eq}}\right)^{2}} \Delta \hat{\boldsymbol{\sigma}}^{\mathrm{r}}: \mathbb{I}^{\mathrm{dev}}: \mathbb{C}^{\mathrm{alg}}-\frac{2}{3} \mu^{\mathrm{s}} \frac{\mathbb{I}^{\mathrm{dev}}:\langle\Delta \varepsilon\rangle^{\mathrm{r}}}{\left(\left(\langle\Delta \varepsilon\rangle^{\mathrm{r}}\right)^{\mathrm{eq}}\right)^{2}}\right] \\
\frac{\partial \mathbb{C}^{\mathrm{SD}}}{\partial\langle\Delta \varepsilon\rangle^{\mathrm{r}}}=(1-D) \frac{\partial \mathbb{C}^{\mathrm{S}}}{\partial\langle\Delta \varepsilon\rangle^{\mathrm{r}}}-\mathbb{C}^{\mathrm{S}} \otimes \frac{\partial D}{\partial\langle\Delta \varepsilon\rangle^{\mathrm{r}}} \\
\frac{\partial \mathbb{C}^{\mathrm{SD}}}{\partial \underline{p}}=-\frac{\partial D}{\partial \underline{p}} \mathbb{C}^{\mathrm{S}}
\end{array}\right.
$$

where $\mathbb{C}^{\text {alg }}$ is given in Appendix $B$. The derivative of the Eshelby tensor $\frac{\partial \mathbb{S}}{\partial\langle\boldsymbol{\varepsilon}\rangle_{0}}=\left(\frac{\partial \mathbb{S}}{\partial\langle\varepsilon\rangle_{0}}+\frac{\partial \mathbb{S}}{\partial D_{0}} \frac{\partial D_{0}}{\partial\langle\varepsilon\rangle_{0}}\right)$ was developed in [80].

Once $\boldsymbol{F}=0$ is satisfied, the effect on the strain increment in each phase of a variation $\mathrm{d} \Delta \varepsilon_{\mathrm{M}}^{\mathrm{r}}$ at constant $\Delta p$ and vice versa can directly be obtained by constraining $\mathrm{d} \boldsymbol{F}=0$ in Eq. (C. $\overline{2})$ leading to

$$
\left\{\begin{array}{l}
\frac{\partial\langle\Delta \varepsilon\rangle_{\mathrm{I}}^{\mathrm{r}}}{\partial \Delta \varepsilon_{\mathrm{M}}^{\mathrm{r}}}=-\mathbb{J}^{-1}: \frac{\partial \boldsymbol{F}}{\partial \Delta \varepsilon_{\mathrm{M}}^{\mathrm{r}}}, \quad \text { and } \frac{\partial\langle\Delta \varepsilon\rangle_{0}^{\mathrm{r}}}{\partial \Delta \varepsilon_{\mathrm{M}}^{\mathrm{r}}}=\frac{1}{v_{0}}\left(\mathbb{I}-v_{\mathrm{I}} \frac{\partial\langle\Delta \varepsilon\rangle_{\mathrm{I}}^{\mathrm{r}}}{\partial \Delta \varepsilon_{\mathrm{M}}^{\mathrm{r}}}\right) \\
\frac{\partial\langle\Delta \varepsilon\rangle_{\mathrm{I}}^{\mathrm{r}}}{\partial \underline{p}}=-\mathbb{J}^{-1}: \frac{\partial \boldsymbol{F}}{\partial \underline{p}}, \text { and } \frac{\partial\langle\Delta \varepsilon\rangle_{0}^{\mathrm{r}}}{\partial \underline{p}}=-\frac{v_{\mathrm{I}}}{v_{0}} \frac{\partial\langle\Delta \varepsilon\rangle_{\mathrm{I}}^{\mathrm{r}}}{\partial \underline{p}}
\end{array}\right.
$$

However, when performing the inverse identification process, the MFH is not related to a finite element formulation in which case there is no existence of a non-local variable $\underline{p}$. In that case, the equations here above simplifies by removing the dependencies in $\underline{p}$, and by performing the following substitution:

$$
\frac{\partial D_{0}}{\partial\langle\Delta \boldsymbol{\varepsilon}\rangle_{0}^{\mathrm{r}}} \rightarrow \frac{\partial D_{0}}{\partial\langle\Delta \boldsymbol{\varepsilon}\rangle_{0}^{\mathrm{r}}}+\frac{\partial D_{0}}{\partial p_{0}} \frac{\partial p_{0}}{\partial\langle\Delta \boldsymbol{\varepsilon}\rangle_{0}^{\mathrm{r}}}=\frac{\partial D_{0}}{\partial\langle\Delta \boldsymbol{\varepsilon}\rangle_{0}^{\mathrm{r}}}+\frac{\partial D_{0}}{\partial p_{0}} \boldsymbol{C}_{0}^{p u}
$$

with the expression of $\boldsymbol{C}_{0}^{p u}$ given in Appendix B. 
Appendix C.1. Linearization of the non-local MFH

At the finite element level, the input of the MFH are the strain increment $\Delta \varepsilon_{\mathrm{M}}^{\mathrm{f}}=\Delta \varepsilon_{\mathrm{M}}^{\mathrm{r}}-\Delta \varepsilon_{\mathrm{M}}^{\text {unload }}$ and the non-local variable $\underline{p}$. The "consistent" linearization of the homogenized stress (38) thus reads

$$
\delta \boldsymbol{\sigma}_{\mathrm{M}}=v_{\mathrm{I}} \delta\langle\boldsymbol{\sigma}\rangle_{\mathrm{I}}+v_{0} \delta\langle\boldsymbol{\sigma}\rangle_{0}=\mathbb{C}_{\mathrm{M}} \delta \Delta \varepsilon_{\mathrm{M}}^{\mathrm{f}}+\boldsymbol{C}_{\mathrm{M}}^{u \underline{p}} \delta \underline{p}=\mathbb{C}_{\mathrm{M}} \delta \Delta \varepsilon_{\mathrm{M}}^{\mathrm{r}}+\boldsymbol{C}_{\mathrm{M}}^{u p} \delta \underline{p} .
$$

The two operators $\mathbb{C}_{\mathrm{M}}$ and $\boldsymbol{C}_{\mathrm{M}}^{u \underline{p}}$ have been evaluated in [80] and read

$$
\begin{aligned}
\mathbb{C}_{\mathrm{M}}= & v_{\mathrm{I}} \mathbb{C}_{\mathrm{I}}^{\text {alg }}: \frac{\partial\langle\Delta \varepsilon\rangle_{\mathrm{I}}^{\mathrm{r}}}{\partial \Delta \varepsilon_{\mathrm{M}}^{\mathrm{r}}}+v_{0}\left(\left(1-D_{0}\right) \mathbb{C}_{0}^{\mathrm{alg}}-\hat{\boldsymbol{\sigma}}_{0} \otimes \frac{\partial D_{0}}{\partial\langle\Delta \varepsilon\rangle_{0}^{\mathrm{r}}}\right): \frac{\partial\langle\Delta \boldsymbol{\varepsilon}\rangle_{0}^{\mathrm{r}}}{\partial \Delta \boldsymbol{\varepsilon}_{\mathrm{M}}^{\mathrm{r}}} \text {, and } \\
\boldsymbol{C}_{\mathrm{M}}^{u \underline{p}}= & v_{\mathrm{I}} \mathbb{C}_{\mathrm{I}}^{\mathrm{alg}}: \frac{\partial\langle\Delta \varepsilon\rangle_{\mathrm{I}}^{\mathrm{r}}}{\partial \underline{p}}+v_{0}\left(\left(1-D_{0}\right) \mathbb{C}_{0}^{\mathrm{alg}}-\hat{\boldsymbol{\sigma}}_{0} \otimes \frac{\partial D_{0}}{\partial\langle\Delta \boldsymbol{\varepsilon}\rangle_{0}^{\mathrm{r}}}\right): \frac{\partial\langle\Delta \boldsymbol{\varepsilon}\rangle_{0}^{\mathrm{r}}}{\partial \underline{p}}- \\
& v_{0} \hat{\boldsymbol{\sigma}}_{0} \frac{\partial D_{0}}{\partial \underline{p}},
\end{aligned}
$$

where the phases algorithmic operators $\mathbb{C}_{i}^{\text {alg }}$ and damage evolution derivatives are given in Appendix B, and where the derivatives of the strain increments in the phases are reported in Appendix C.

The linearization of the homogenized equivalent plastic strain in the matrix $p_{0}$ reads, see [80]

$$
\delta p_{0}=\frac{1}{h} \boldsymbol{N}: \mathbb{C}_{0}^{\mathrm{el}}: \delta\langle\varepsilon\rangle_{0}=\frac{2 \mu}{h} \boldsymbol{N}:\left(\frac{\partial\langle\varepsilon\rangle_{0}}{\partial \Delta \varepsilon_{\mathrm{M}}^{\mathrm{f}}}: \delta \Delta \varepsilon_{\mathrm{M}}^{\mathrm{f}}+\frac{\partial\langle\varepsilon\rangle_{0}}{\partial \underline{p}} \delta \underline{p}\right),
$$

yielding

$$
\begin{aligned}
\boldsymbol{C}_{\mathrm{M}}^{p u} & =\frac{\partial p_{0}}{\partial \Delta \varepsilon_{\mathrm{M}}^{\mathrm{f}}}=\frac{2 \mu}{h} \boldsymbol{N}: \frac{\partial\langle\varepsilon\rangle_{0}}{\partial \Delta \varepsilon_{\mathrm{M}}^{\mathrm{f}}}, \\
C_{\mathrm{M}}^{p \underline{p}} & =\frac{\partial p_{0}}{\partial \underline{p}}=\frac{2 \mu}{h} \boldsymbol{N}: \frac{\partial\langle\varepsilon\rangle_{0}}{\partial \underline{p}} .
\end{aligned}
$$

However, when performing the inverse identification process, the MFH is not related to a finite element formulation in which case there is no existence of a non-local variable $p$. The homogenization tensor $\mathbb{C}_{\mathrm{M}}$ should thus also include the part resulting from the derivative of the damage variable with respect to the local internal variable:

$$
\begin{aligned}
\mathbb{C}_{\mathrm{M}}= & v_{0}\left[(1-D) \mathbb{C}_{0}^{\mathrm{alg}}-\hat{\boldsymbol{\sigma}}_{0} \otimes\left(\frac{\partial D_{0}}{\partial\langle\Delta \varepsilon\rangle_{0}^{\mathrm{r}}}+\frac{\partial D_{0}}{\partial p} \boldsymbol{C}_{0}^{p u}\right)\right]: \frac{\partial\langle\Delta \boldsymbol{\varepsilon}\rangle_{0}^{\mathrm{r}}}{\partial \Delta \boldsymbol{\varepsilon}_{\mathrm{M}}^{\mathrm{r}}}+ \\
& v_{\mathrm{I}} \mathbb{C}_{\mathrm{I}}^{\mathrm{alg}}: \frac{\partial\langle\Delta \boldsymbol{\varepsilon}\rangle_{\mathrm{I}}^{\mathrm{r}}}{\partial \Delta \boldsymbol{\varepsilon}_{\mathrm{M}}^{\mathrm{r}}}
\end{aligned}
$$




\section{Appendix D. Data-driven probability sampling}

The random samples generating process is carried out following the detailed three sequential sub-processes:

1. Preparation of data-set for random samples generator. The original dataset $\mathbf{b}_{i},(i=1, \ldots, N)$ is normalized by removing the mean and scaling it to the unit variance. The mean vector of $\mathbb{E}[\mathbf{b}]$ is estimated statistically by

$$
\mathbb{E}[\mathbf{b}]=\frac{1}{N} \sum_{i=1}^{N} \mathbf{b}_{i}
$$

and the empirical estimate of covariance matrix $\mathbb{E}\left\{(\mathbf{b}-\mathbb{E}[\mathbf{b}])(\mathbf{b}-\mathbb{E}[\mathbf{b}])^{\mathrm{T}}\right\}$ reads,

$$
[\mathbf{C}]=\frac{1}{N-1} \sum_{i=1}^{N}\left(\mathbf{b}_{i}-\mathbb{E}[\mathbf{b}]\right)\left(\mathbf{b}_{i}-\mathbb{E}[\mathbf{b}]\right)^{\mathrm{T}} .
$$

The eigen-decomposition of matrix $[\mathbf{C}]$ gives

$$
[\mathbf{C}]=[\boldsymbol{Q}][\boldsymbol{\xi}][\boldsymbol{Q}]^{\mathrm{T}},
$$

with the matrix $[Q]=\left[Q_{1}, \ldots, Q_{n}\right]$, where $\boldsymbol{Q}_{k}$ is the $k^{\text {th }}$ eigenvector of $[\mathbf{C}]$ corresponding to the $k^{\text {th }}$ eigenvalue $\xi_{k}$, and the matrix $[\boldsymbol{\xi}]=\operatorname{diag}\left[\xi_{1}, \ldots, \xi_{n}\right]$. The normalized dataset is then computed by

$$
\boldsymbol{\eta}_{i}=[\boldsymbol{\xi}]^{-1 / 2}[\boldsymbol{Q}]^{\mathrm{T}}\left(\boldsymbol{b}_{i}-\mathbb{E}[\mathbf{b}]\right), \quad \forall i=1, \ldots, N
$$

The original data-set $[\boldsymbol{b}]_{n \times N}=\left[\boldsymbol{b}_{1}, \ldots, \boldsymbol{b}_{N}\right]$ is thus transferred to $[\boldsymbol{\eta}]_{n \times N}=$ $\left[\boldsymbol{\eta}_{1}, \ldots, \boldsymbol{\eta}_{N}\right]$.

2. Random data generating process. The following steps have to be carried out.

- The given data-set $\boldsymbol{\eta}_{i},(i=1, \ldots, N)$, serves as $N$ realizations of a random vector $\boldsymbol{V}$, which is defined on a probability space $(\Theta, \mathcal{T}, \mathcal{P})$, with value in $\mathbb{R}^{n}$. The non-parametric estimate of the probability distribution function (pdf) $p_{\boldsymbol{V}}$ can be constructed by using the Gaussian kernel-density estimation method, with

$$
p_{\boldsymbol{V}}(\boldsymbol{\eta})=\frac{1}{N} \sum_{i=1}^{N} \frac{1}{\left(\sqrt{2 \pi} \hat{s}_{n}\right)^{n}} \exp \left(-\frac{1}{2 \hat{s}_{n}^{2}}\left\|\frac{\hat{s}_{n}}{s_{n}} \boldsymbol{\eta}_{i}-\boldsymbol{\eta}\right\|^{2}\right)
$$


with $\|\boldsymbol{\eta}\|^{2}=\eta_{1}^{2}+\ldots+\eta_{n}^{2}$, and where the multidimensional optimal Silverman bandwith $s_{n}$ and parameter $\hat{s}_{n}$ read

$$
s_{n}=\left[\frac{4}{N(2+n)}\right]^{1 /(4+n)}, \quad \hat{s}_{n}=\frac{s_{n}}{\sqrt{s_{n}^{2}+\frac{N-1}{N}}} .
$$

- Based on the normalized observation data-set $[\boldsymbol{\eta}]_{n \times N}$, a diffusion-map basis $[\boldsymbol{\phi}]$ is constructed using the Gaussian kernel, which is defined on $\mathbb{R}^{n} \times \mathbb{R}^{n}$, by

$$
k_{\epsilon}\left(\boldsymbol{\eta}_{i}, \boldsymbol{\eta}_{j}\right)=\exp \left(-\frac{1}{4 \epsilon}\left\|\boldsymbol{\eta}_{i}-\boldsymbol{\eta}_{j}\right\|^{2}\right), \quad \forall i, j=1, \ldots, N
$$

Let $[\boldsymbol{K}]$ be the symmetric matrix in $\mathbb{M}_{N}$, with its entries $[\boldsymbol{K}]_{i j}=$ $k_{\epsilon}\left(\boldsymbol{\eta}_{i}, \boldsymbol{\eta}_{j}\right)$. A diagonal real matrix $[\boldsymbol{\rho}]=\operatorname{diag}\left(\rho_{1}, \ldots, \rho_{N}\right)$, in $\mathbb{M}_{N}$, is first computed by

$$
\rho_{i}=\sum_{j=1}^{N}[K]_{i j}, \quad \forall i, j=1, \ldots, N
$$

A symmetric matrix $\left[\mathbb{P}_{\mathrm{s}}\right]$ is then defined as

$$
\left[\mathbb{P}_{\mathrm{s}}\right]=[\boldsymbol{\rho}]^{-1 / 2}[\boldsymbol{K}][\boldsymbol{\rho}]^{-1 / 2},
$$

with its eigenvalue problem defined by $\left[\mathbb{P}_{\mathrm{s}}\right] \psi^{i}=\lambda_{i} \psi^{i},(i=1, \ldots, N)$. Using the definition of $\left[\mathbb{P}_{\mathrm{s}}\right]$, Eq. (D.9), and multiplying by $[\boldsymbol{\rho}]^{-1 / 2}$ its corresponding eigenvalue problem, give

$$
[\boldsymbol{\rho}]^{-1}[\boldsymbol{K}][\boldsymbol{\rho}]^{-1 / 2} \boldsymbol{\psi}^{i}=\lambda_{i}[\boldsymbol{\rho}]^{-1 / 2} \boldsymbol{\psi}^{i}
$$

which corresponds to the new eigenvalue problem,

$$
[\mathbb{P}] \zeta^{i}=\lambda_{i} \zeta^{i}, \quad i=1, \ldots, N
$$

where $[\mathbb{P}]=[\boldsymbol{\rho}]^{-1}[\boldsymbol{K}]$. The eigenvalues and eigenvectors of this new problem are respectively $\lambda_{i}$ and $\zeta^{i}=[\boldsymbol{\rho}]^{-1 / 2} \boldsymbol{\psi}^{i}$. From the definition of the diagonal matrix $[\boldsymbol{\rho}]$, Eq. (D.8), it derives that $\sum_{j=1}^{N}[\mathbb{P}]_{i j}=1$, $\forall i=1, \ldots, N$. Therefore, it can be seen as a Markov chain transition matrix in one step. 
Finally, the diffusion map basis is defined by $[\phi]=\left[\phi^{1}, \ldots, \phi^{N}\right] \in \mathbb{M}_{N, N}$, such that

$$
\phi^{i}=\lambda_{i} \zeta^{i} \in \mathbb{R}^{N}, \quad \forall i=1, \ldots, N,
$$

where $\lambda_{i}$ are the eigenvalues of the matrix $\left[\mathbb{P}_{\mathrm{s}}\right]$. Assuming the eigenvalues are ordered in a descending order, it can be proved that

$$
1=\lambda_{1} \geq \lambda_{2} \geq \ldots \geq \lambda_{N} .
$$

A dimension reduction can be achieved by discarding the basis vectors corresponding to the eigenvalues which are lower than a threshold value $\tau$, such as

$$
\tau \geq \lambda_{m+1} \geq \lambda_{m+2} \geq \ldots \geq \lambda_{N} .
$$

The reduced basis $[\phi]_{\mathrm{r}}=\left[\phi^{1}, \ldots, \boldsymbol{\phi}^{m}\right] \in \mathbb{M}_{N, m}$, is then obtained.

Remembering that the given data-set $[\boldsymbol{\eta}]_{n \times N}$ serves as a realization of the random matrix $[\boldsymbol{V}]$, which is defined on the probability space $(\Theta, \mathcal{T}, \mathcal{P})$, with value in $\mathbb{M}_{n, N}$, this random matrix $[\boldsymbol{V}]$ can be represented with the reduced diffusion map basis $[\boldsymbol{\phi}]_{\mathrm{r}}$ as

$$
[\boldsymbol{V}]=[\boldsymbol{Z}][\boldsymbol{\phi}]_{\mathrm{r}}^{\mathrm{T}},
$$

and it yields

$$
[\boldsymbol{Z}]=[\boldsymbol{V}][\boldsymbol{a}] \text {, and }[\boldsymbol{a}]=[\boldsymbol{\phi}]_{\mathrm{r}}\left([\boldsymbol{\phi}]_{\mathrm{r}}^{\mathrm{T}}[\boldsymbol{\phi}]_{\mathrm{r}}\right)^{-1} \in \mathbb{M}_{N, m} .
$$

In particular, using the given data-set $[\boldsymbol{\eta}]_{n \times N}$ yields

$$
[\boldsymbol{z}]=[\boldsymbol{\eta}][\boldsymbol{a}] \in \mathbb{M}_{n, m} .
$$

Remark: If the order reduction is not desired in the random vector generating process, the step of diffusion map basis construction is not necessary anymore. One can use $[\boldsymbol{a}]=[\boldsymbol{I}]_{N \times N}, m=N$ and $[\boldsymbol{\phi}]_{\mathrm{r}}=[\boldsymbol{\phi}]=$ $[\boldsymbol{I}]_{N \times N}$ in the following steps.

- The additional realizations of the random matrix $[\boldsymbol{V}]$ are computed through Eq. (D.15) with additional realizations of $[\boldsymbol{Z}]$, which are generated by solving a Itô stochastic differential equations (ISDE). It is defined as a Markov stochastic process $\left\{([\mathcal{Z}(r)],[\mathcal{Y}(r)]), r \in \mathbb{R}^{+}\right\}$on 
the probability space $(\Theta, \mathcal{T}, \mathcal{P})$, with value in $\mathbb{M}_{n, N} \times \mathbb{M}_{n, N}$, indexed by $\mathbb{R}^{+}=[0,+\infty)$. The ISDE is defined as

$$
\begin{aligned}
\mathrm{d}[\mathcal{Z}(r)] & =[\mathcal{Y}(r)] \mathrm{d} r, \\
\mathrm{~d}[\mathcal{Y}(r)] & =[\mathcal{L}([\mathcal{Z}(r)])] \mathrm{d} r-\frac{1}{2} f_{0}[\mathcal{Y}(r)] \mathrm{d} r+\sqrt{f_{0}}[\mathrm{~d} \mathcal{W}(r)],
\end{aligned}
$$

with the initial conditions

$$
\left[\mathcal{Z}_{0}\right]=[\boldsymbol{z}]=[\boldsymbol{\eta}][\boldsymbol{a}], \quad\left[\mathcal{Y}_{0}\right]=[\mathcal{N}][\boldsymbol{a}], \quad\left[\mathcal{W}_{0}\right]=[\mathbf{0}]_{n \times m},
$$

where $[\mathcal{N}]_{n \times N}$ represents the random matrix whose entries are $n \times N$ independent random variables with normalized Gaussian distribution, and $[\mathrm{d} \mathcal{W}(r)]_{n \times m}=[\mathrm{d} \boldsymbol{W}(r)][\boldsymbol{a}]$ with $[\mathrm{d} \boldsymbol{W}(r)]_{n \times N}$ consisting of a $n \times N$ independent normalized Wiener process.

The term $[\mathcal{L}([\mathcal{Z}(r)])]_{n \times m}$ in Eq. (D.19) is expressed as

$$
[\mathcal{L}([\mathcal{Z}(r)])]=\left[\boldsymbol{L}\left([\mathcal{Z}(r)][\boldsymbol{\phi}]_{\mathrm{r}}^{\mathrm{T}}\right)\right][\boldsymbol{a}] .
$$

We note $[\boldsymbol{q}]=[\mathcal{Z}(r)][\boldsymbol{\phi}]_{\mathrm{r}}^{\mathrm{T}}$, in which $[\boldsymbol{q}]=\left[\boldsymbol{q}_{1}, \ldots, \boldsymbol{q}_{N}\right]$ with $\boldsymbol{q}_{i} \in \mathbb{R}^{n}$. The columns of the matrix $[\boldsymbol{L}([\boldsymbol{q}])]_{n \times N}$ read

$$
[\boldsymbol{L}([\boldsymbol{q}])]_{:, i}=-\left.\frac{\partial \mathcal{V}(\mathbf{g})}{\partial \mathbf{g}}\right|_{\mathbf{g}=\boldsymbol{q}_{i}}, \quad \forall i=1, \ldots, N,
$$

where the potential $\mathcal{V}(\mathbf{g})$ is defined on $\mathbb{R}^{n}$ with the values in $\mathbb{R}^{+}$, and reads

$$
\mathcal{V}(\mathbf{g})=-\log \left[\frac{1}{N} \sum_{j=1}^{N} \exp \left(-\frac{1}{2 \hat{s}_{n}^{2}}\left\|\frac{\hat{s}_{n}}{s_{n}} \boldsymbol{\eta}_{j}-\mathbf{g}\right\|^{2}\right)\right]
$$

In this last equation, $\boldsymbol{\eta}_{j}, \forall j=1, \ldots, N$ are column vectors of the given data-set $[\boldsymbol{\eta}]$, and the parameters $\hat{s}_{n}$ and $s_{n}$ are defined in Eq. (D.6).

- To be complete, the ISDE presented in Eqs. (D.18-D.19) is solved with the Störmer-Verlet scheme. The continuous index parameter $r$ is discretized into $r_{0}, r_{1}, \ldots, r_{l}, \ldots$ with the constant sampling step $\Delta r$ and $r_{l}=l \Delta r$. Using the following notations $\left[\mathcal{Z}_{l}\right]=\left[\mathcal{Z}\left(r_{l}\right)\right],\left[\mathcal{Y}_{l}\right]=\left[\mathcal{Y}\left(r_{l}\right)\right]$ 
and $\left[\mathcal{W}_{l}\right]=\left[\mathcal{W}\left(r_{l}\right)\right]$, the Störmer-Verlet scheme yields

$$
\begin{aligned}
{\left[\mathcal{Z}_{l+\frac{1}{2}}\right] } & =\left[\mathcal{Z}_{l}\right]+\frac{\Delta r}{2}\left[\mathcal{Y}_{l}\right] \\
{\left[\mathcal{Y}_{l+1}\right] } & =\frac{1-\alpha}{1+\alpha}\left[\mathcal{Y}_{l}\right]+\frac{\Delta r}{1+\alpha}\left[\mathcal{L}_{l+\frac{1}{2}}\right]+\frac{\sqrt{f_{0}}}{1+\alpha}\left[\Delta \mathcal{W}_{l+1}\right] \\
{\left[\mathcal{Z}_{l+1}\right] } & =\left[\mathcal{Z}_{l+\frac{1}{2}}\right]+\frac{\Delta r}{2}\left[\mathcal{Y}_{l+1}\right]
\end{aligned}
$$

where $\left[\mathcal{L}_{l+\frac{1}{2}}\right]=\left[\mathcal{L}\left(\left[\mathcal{Z}_{l+\frac{1}{2}}\right]\right)\right]$ and $\alpha=f_{0} \Delta r / 4$.

The additional realizations of $[\boldsymbol{Z}]$ are sampled after every $M_{0}$ steps. Let $[\hat{\boldsymbol{z}}]^{k}$ denote the additional realizations of $\boldsymbol{Z}$, which take the value of $\left[\mathcal{Z}_{l}\right]$ at $l=k M_{0}$ with $k=1, \ldots, n_{\mathrm{MC}}$, where $n_{\mathrm{MC}}$ is the number of the required additional realizations and $M_{0}$ is a positive integer sampling parameter. Hence, the additional realizations of the random matrix $\boldsymbol{V}$ are obtained by rewriting Eq. (D.15) as

$$
[\hat{\boldsymbol{\eta}}]^{k}=[\hat{\boldsymbol{z}}]^{k}[\phi]_{\mathrm{r}}^{\mathrm{T}} \in \mathbb{M}_{n, N}, \quad \forall k=1, \ldots, n_{\mathrm{MC}}
$$

3. Recovering generated data in the original data-set scale. According to the original data-set normalization Eq. (D.4), the generated random data-set $[\hat{\boldsymbol{\eta}}]^{k}=\left[\hat{\boldsymbol{\eta}}_{1}^{k}, \ldots, \hat{\boldsymbol{\eta}}_{N}^{k}\right]$, with $k=1, \ldots, n_{\mathrm{MC}}$, can be transferred back to recover the scale and mean of the original data-set by

$$
\hat{\boldsymbol{b}}_{i}=\mathbb{E}(\mathbf{b})+[\boldsymbol{Q}][\boldsymbol{\xi}]^{1 / 2} \hat{\boldsymbol{\eta}}_{i}^{k}, \quad \forall i=1, \ldots, N
$$

Finally, $k \times N$ additional realizations of $\beta^{D}$ are generated.

Table D.4: Parameters used in the sampling process.

\begin{tabular}{ccccccc}
\hline$n$ & $N$ & $\epsilon$ & $f_{0}$ & $F a c$ & $\Delta r$ & $M_{0}$ \\
\hline 11 & 1868 & 2.7318 & 1.5 & 20 & 0.1534 & 100 \\
\hline
\end{tabular}

Practically, in this work, the order reduction is not applied, and the parameters used in the process are given in Table D.4, where the value of $\Delta r$, which is used in Eq. (D.24), is computed by $\Delta r=2 \pi \hat{s}_{n} / F a c$. More details can be found in [60]. 


\section{Acknowledgment}

The research has been funded by the Walloon Region under the agreement no 1410246-STOMMMAC (CT-INT 2013-03-28) in the context of the MERA.NET Joint Call 2014.

\section{References}

[1] L. Graham-Brady, S. Arwade, D. Corr, M. Gutirrez, D. Breysse, M. Grigoriu, N. Zabaras, Probability and Materials: from Nano- to Macro-Scale: A summary, Probabilistic Engineering Mechanics 21 (3) (2006) 193 - 199, ISSN 0266-8920, doi:https://doi.org/10.1016/j.probengmech.2005.10.005, URL http://www.sciencedirect.com/science/article/pii/S0266892005000706, probability and Materials: from Nano- to Macro-Scale.

[2] D. L. McDowell, A perspective on trends in multiscale plasticity, International Journal of Plasticity 26 (9) (2010) 1280 - 1309, ISSN 0749-6419, doi:https://doi.org/10.1016/j.ijplas.2010.02.008, URL http://www.sciencedirect.com/science/article/pii/S0749641910000306, special Issue In Honor of David L. McDowell.

[3] R. Ghanem, P. Spanos, Stochastic Finite Elements: A Spectral Approach, Springer Verlag, 1991.

[4] G. Stefanou, The stochastic finite element method: Past, present and future, Computer Methods in Applied Mechanics and Engineering 198 (912) (2009) 1031 - 1051, ISSN 0045-7825, doi: http://dx.doi.org/10.1016/j.cma.2008.11.007.

[5] D. Charmpis, G. Schuller, M. Pellissetti, The need for linking micromechanics of materials with stochastic finite elements: A challenge for materials science, Computational Materials Science 41 (1) (2007) 27 - 37, ISSN 0927-0256, doi:https://doi.org/10.1016/j.commatsci.2007.02.014, URL http://www.sciencedirect.com/science/article/pii/S0927025607000602.

[6] K. Alzebdeh, M. Ostoja-Starzewski, Micromechanically based stochastic finite elements: length scales and anisotropy, Probabilistic Engineering Mechanics 11 (4) (1996) 205 - 214, ISSN 0266-8920, doi: 
http://dx.doi.org/10.1016/0266-8920(96)00015-X, third International Stochastic Structural Dynamics Conference.

[7] M. Ostoja-Starzewski, X. Wang, Stochastic finite elements as a bridge between random material microstructure and global response, Computer Methods in Applied Mechanics and Engineering 168 (14) (1999) 35 - 49, ISSN 0045-7825, doi:http://dx.doi.org/10.1016/S0045-7825(98)00105-4.

[8] M. Blacklock, H. Bale, M. Begley, B. Cox, Generating virtual textile composite specimens using statistical data from micro-computed tomography: 1D tow representations for the Binary Model, Journal of the Mechanics and Physics of Solids 60 (3) (2012) 451 - 470, ISSN 0022-5096, doi:http://dx.doi.org/10.1016/j.jmps.2011.11.010, URL http://www.sciencedirect.com/science/article/pii/S0022509611002225.

[9] D. Tal, J. Fish, Generating a statistically equivalent representative volume element with discrete defects, Composite Structures 153 (2016) 791 - 803, ISSN 0263-8223, doi:http://dx.doi.org/10.1016/j.compstruct.2016.06.077, URL http://www.sciencedirect.com/science/article/pii/S0263822316310790.

[10] A. Vanaerschot, B. N. Cox, S. V. Lomov, D. Vandepitte, Stochastic multi-scale modelling of textile composites based on internal geometry variability, Computers \& Structures 122 (2013) 55 - 64, ISSN 00457949, doi:http://dx.doi.org/10.1016/j.compstruc.2012.10.026, URL http://www.sciencedirect.com/science/article/pii/S0045794912002647, computational Fluid and Solid Mechanics 2013.

[11] A. Gupta, A. Cecen, S. Goyal, A. K. Singh, S. R. Kalidinhi, Structure-property linkages using a data science approach: Application to a non-metallic inclusion/steel composite system, Acta Materialia 91 (2015) 239 - 254, ISSN 13596454, doi:http://dx.doi.org/10.1016/j.actamat.2015.02.045, URL http://www.sciencedirect.com/science/article/pii/S1359645415001603.

[12] T. Vaughan, C. McCarthy, A combined experimental-numerical approach for generating statistically equivalent fibre distributions for high strength laminated composite materials, Composites 
Science and Technology 70 (2) (2010) 291 - 297, ISSN 02663538, doi:http://dx.doi.org/10.1016/j.compscitech.2009.10.020, URL http://www. sciencedirect.com/science/article/pii/S0266353809003832.

[13] A. Melro, P. Camanho, S. Pinho, Generation of random distribution of fibres in long-fibre reinforced composites, Composites Science and Technology 68 (9) (2008) 2092 - 2102, ISSN 02663538, doi:http://dx.doi.org/10.1016/j.compscitech.2008.03.013, URL http://www.sciencedirect.com/science/article/pii/S0266353808001048.

[14] L. Wu, C. N. Chung, Z. Major, L. Adam, L. Noels, From SEM images to elastic responses: A stochastic multiscale analysis of UD fiber reinforced composites, Composite Structures 189 (2018) 206 - 227, ISSN 0263-8223, doi:https://doi.org/10.1016/j.compstruct.2018.01.051, URL http://www.sciencedirect.com/science/article/pii/S0263822317327770.

[15] P. Kanouté, D. Boso, J. Chaboche, B. Schrefler, Multiscale Methods for Composites: A Review, Archives of Computational Methods in Engineering 16 (2009) 31-75, ISSN 1134-3060, 10.1007/s11831-008-9028-8.

[16] L. Noels, L. Wu, L. Adam, J. Seyfarth, G. Soni, J. Segurado, G. Laschet, G. Chen, M. Lesueur, M. Lobos, T. Böhlke, T. Reiter, S. Oberpeilsteiner, D. Salaberger, D. Weichert, C. Broeckmann, Effective Properties, in: Handbook of Software Solutions for ICME, Wiley-VCH Verlag GmbH \& Co. KGaA, ISBN 9783527693566, 433-485, doi:10.1002/9783527693566.ch6, URL http://dx.doi.org/10.1002/9783527693566.ch6, 2016.

[17] K. Matous, M. G. Geers, V. G. Kouznetsova, A. Gillman, A review of predictive nonlinear theories for multiscale modeling of heterogeneous materials, Journal of Computational Physics 330 (Supplement C) (2017) 192 - 220, ISSN 0021-9991, doi:10.1016/j.jcp.2016.10.070.

[18] F. Feyel, Multiscale FE2 elastoviscoplastic analysis of composite structures, Computational Materials Science 16 (1) (1999) 344 - 354, ISSN 0927-0256, doi:http://dx.doi.org/10.1016/S0927-0256(99)00077-4, URL http://www. sciencedirect. com/science/article/pii/S0927025699000774.

[19] J. Michel, H. Moulinec, P. Suquet, Effective properties of composite materials with periodic microstructure: a compu- 
tational approach, Computer Methods in Applied Mechanics and Engineering 172 (1) (1999) 109 - 143, ISSN 00457825, doi:http://dx.doi.org/10.1016/S0045-7825(98)00227-8, URL http://www.sciencedirect.com/science/article/pii/S0045782598002278.

[20] K. Terada, M. Hori, T. Kyoya, N. Kikuchi, Simulation of the multi-scale convergence in computational homogenization approaches, International Journal of Solids and Structures 37 (16) (2000) 2285 - 2311, ISSN 0020-7683, doi:http://dx.doi.org/10.1016/S0020-7683(98)00341-2, URL http://www.sciencedirect.com/science/article/pii/S0020768398003412.

[21] V. Kouznetsova, W. A. M. Brekelmans, F. P. T. Baaijens, An approach to micro-macro modeling of heterogeneous materials, Computational Mechanics 27 (1) (2001) 37-48, ISSN 1432-0924, doi:10.1007/s004660000212, URL https://doi.org/10.1007/s004660000212.

[22] C. Miehe, Strain-driven homogenization of inelastic microstructures and composites based on an incremental variational formulation, International Journal for Numerical Methods in Engineering 55 (11) (2002) 1285-1322, ISSN 1097-0207, doi:10.1002/nme.515, URL http://dx.doi.org/10.1002/nme.515.

[23] M. Ostoja-Starzewski, X. Du, Z. F. Khisaeva, W. Li, Comparisons of the Size of the Representative Volume Element in Elastic, Plastic, Thermoelastic, and Permeable Random Microstructures, International Journal for Multiscale Computational Engineering 5 (2) (2007) 73-82, ISSN 1543-1649.

[24] M. Salmi, F. Auslender, M. Bornert, M. Fogli, Apparent and effective mechanical properties of linear matrix-inclusion random composites: Improved bounds for the effective behavior, International Journal of Solids and Structures 49 (10) (2012) 1195 - 1211, ISSN 0020-7683, doi:https://doi.org/10.1016/j.ijsolstr.2012.01.018, URL http://www.sciencedirect.com/science/article/pii/S0020768312000340.

[25] P. Trovalusci, M. Ostoja-Starzewski, M. L. De Bellis, A. Murrali, Scale-dependent homogenization of random composites as micropolar continua, European Journal of Mechanics 
- A/Solids 49 (0) (2015) 396 - 407, ISSN 0997-7538, doi: http://dx.doi.org/10.1016/j.euromechsol.2014.08.010.

[26] E. Reccia, M. L. De Bellis, P. Trovalusci, R. Masiani, Sensitivity to material contrast in homogenization of random particle composites as micropolar continua, Composites Part B: Engineering 136 (2018) 39 - 45, ISSN 13598368, doi:https://doi.org/10.1016/j.compositesb.2017.10.017, URL http://www.sciencedirect.com/science/article/pii/S1359836817325088.

[27] M. Ostoja-Starzewski, Scale effects in plasticity of random media: status and challenges, International Journal of Plasticity $21 \quad(6) \quad(2005) \quad 1119$ - 1160 , ISSN 07496419, doi:https://doi.org/10.1016/j.ijplas.2004.06.008, URL http://www.sciencedirect.com/science/article/pii/S0749641904001391, plasticity of Multiphase Materials.

[28] A. Hachemi, M. Chen, G. Chen, D. Weichert, Limit state of structures made of heterogeneous materials, International Journal of Plasticity $63 \quad(2014) \quad 124 \quad-\quad 137, \quad$ ISSN $0749-$ 6419, doi:https://doi.org/10.1016/j.ijplas.2014.03.019, URL http://www.sciencedirect.com/science/article/pii/S0749641914000862, deformation Tensors in Material Modeling in Honor of Prof. Otto T. Bruhns.

[29] V. Lucas, J.-C. Golinval, S. Paquay, V.-D. Nguyen, L. Noels, L. Wu, A stochastic computational multiscale approach; Application to MEMS resonators, Computer Methods in Applied Mechanics and Engineering 294 (2015) 141 - 167, ISSN 0045-7825, doi:http://dx.doi.org/10.1016/j.cma.2015.05.019, URL http://www.sciencedirect.com/science/article/pii/S0045782515001929.

[30] D. Savvas, G. Stefanou, M. Papadrakakis, Determination of RVE size for random composites with local volume fraction variation, Computer Methods in Applied Mechanics and Engineering 305 (2016) 340 - 358, ISSN 0045-7825, doi:http://dx.doi.org/10.1016/j.cma.2016.03.002, URL http://www.sciencedirect.com/science/article/pii/S0045782516300822.

[31] G. Stefanou, D. Savvas, M. Papadrakakis, Stochastic finite element analysis of composite structures based on mesoscale ran- 
dom fields of material properties, Computer Methods in Applied Mechanics and Engineering 326 (2017) 319 - 337, ISSN 0045-7825, doi:https://doi.org/10.1016/j.cma.2017.08.002, URL http://www.sciencedirect.com/science/article/pii/S0045782517305868.

[32] L. Wu, V. Lucas, V.-D. Nguyen, J.-C. Golinval, S. Paquay, L. Noels, A stochastic multi-scale approach for the modeling of thermoelastic damping in micro-resonators, Computer Methods in Applied Mechanics and Engineering 310 (2016) 802 - 839, ISSN 0045-7825, doi:http://dx.doi.org/10.1016/j.cma.2016.07.042, URL http://www. sciencedirect.com/science/article/pii/S0045782516303930.

[33] D. Pivovarov, P. Steinmann, Modified SFEM for computational homogenization of heterogeneous materials with microstructural geometric uncertainties, Computational Mechanics 57 (1) (2016) 123-147.

[34] J. Yvonnet, Q.-C. He, The reduced model multiscale method (R3M) for the non-linear homogenization of hyperelastic media at finite strains, Journal of Computational Physics 223 (1) (2007) 341 - 368, ISSN 0021-9991, doi:https://doi.org/10.1016/j.jcp.2006.09.019, URL http://www.sciencedirect.com/science/article/pii/S0021999106004402.

[35] A. Radermacher, B. A. Bednarcyk, B. Stier, J. Simon, L. Zhou, S. Reese, Displacement-based multiscale modeling of fiber-reinforced composites by means of proper orthogonal decomposition, Advanced Modeling and Simulation in Engineering Sciences 3 (1) (2016) 29, ISSN 2213-7467, doi:10.1186/s40323-016-0082-8, URL https://doi .org/10.1186/s40323-016-0082-8.

[36] J. Hernández, J. Oliver, A. Huespe, M. Caicedo, J. Cante, High-performance model reduction techniques in computational multiscale homogenization, Computer Methods in Applied Mechanics and Engineering 276 (2014) 149 - 189, ISSN 0045-7825, doi:https://doi.org/10.1016/j.cma.2014.03.011, URL http://www. sciencedirect.com/science/article/pii/S0045782514000978.

[37] D. Soldner, B. Brands, R. Zabihyan, P. Steinmann, J. Mergheim, A numerical study of different projection-based model reduction techniques applied to computational homogenisation, Computational Mechanics 
60 (4) (2017) 613-625, ISSN 1432-0924, doi:10.1007/s00466-017-1428$\mathrm{x}$, URL https://doi.org/10.1007/s00466-017-1428-x.

[38] J.-C. Michel, P. Suquet, Nonuniform transformation field analysis: a reduced model for multiscale nonlinear problems in solid mechanics, in: U. Galvanetto, F. Aliabadi (Eds.), Multiscale Modelling in Solid Mechanics - Computational Approaches, Imperial College Press, Imperial College Press, London., 159-206, URL https://hal . archives-ouvertes .fr/hal-00367772, iSBN: 9781-84816-307-2., 2009.

[39] J.-C. Michel, P. Suquet, A model-reduction approach in micromechanics of materials preserving the variational structure of constitutive relations, Journal of the Mechanics and Physics of Solids 90 (2016) 254 - 285, ISSN 0022-5096, doi:https://doi.org/10.1016/j.jmps.2016.02.005, URL http://www.sciencedirect.com/science/article/pii/S0022509616300928.

[40] F. Fritzen, M. Leuschner, Reduced basis hybrid computational homogenization based on a mixed incremental formulation, Computer Methods in Applied Mechanics and Engineering 260 (2013) 143 - 154, ISSN 0045-7825, doi:https://doi.org/10.1016/j.cma.2013.03.007, URL http://www.sciencedirect.com/science/article/pii/S0045782513000583.

[41] F. Fritzen, M. Hodapp, The finite element square reduced (FE2R) method with GPU acceleration: towards three-dimensional twoscale simulations, International Journal for Numerical Methods in Engineering 107 (10) (2016) 853-881, doi:10.1002/nme.5188, URL https://onlinelibrary.wiley.com/doi/abs/10.1002/nme.5188.

[42] S. Wulfinghoff, F. Cavaliere, S. Reese, Model order reduction of nonlinear homogenization problems using a HashinShtrikman type finite element method, Computer Methods in Applied Mechanics and Engineering 330 (2018) 149 - 179, ISSN 0045-7825, doi:https://doi.org/10.1016/j.cma.2017.10.019, URL http://www.sciencedirect.com/science/article/pii/S0045782517306904.

[43] Z. Liu, M. Bessa, W. K. Liu, Self-consistent clustering analysis: An efficient multi-scale scheme for inelastic heterogeneous materials, Computer Methods in Applied Mechanics and Engineering 306 (2016) 319 - 341, 
ISSN 0045-7825, doi:https://doi.org/10.1016/j.cma.2016.04.004, URL http://www. sciencedirect.com/science/article/pii/S0045782516301499.

[44] D. Wirtz, N. Karajan, B. Haasdonk, Surrogate modeling of multiscale models using kernel methods, International Journal for Numerical Methods in Engineering 101 (1) (2015) 1-28, ISSN 1097-0207, doi: 10.1002/nme.4767, URL http://dx.doi.org/10.1002/nme. 4767.

[45] G. Perrin, C. Soize, D. Duhamel, C. Funfschilling, Identification of Polynomial Chaos Representations in High Dimension from a Set of Realizations, SIAM Journal on Scientific Computing 34 (6) (2012) A2917-A2945, doi:10.1137/11084950X, URL https://doi.org/10.1137/11084950X.

[46] J. Fish, W. Wu, A nonintrusive stochastic multiscale solver, International Journal for Numerical Methods in Engineering 88 (9) (2011) 862 879, ISSN 1097-0207, doi:10.1002/nme.3201.

[47] A. Clément, C. Soize, J. Yvonnet, Computational nonlinear stochastic homogenization using a nonconcurrent multiscale approach for hyperelastic heterogeneous microstructures analysis, International Journal for Numerical Methods in Engineering 91 (8) (2012) 799-824, ISSN 10970207, doi:10.1002/nme.4293.

[48] J. Yvonnet, E. Monteiro, Q.-C. He, Computational homogenization method and reduced database model for hyperelastic heterogeneous structures, International Journal for Multiscale Computational Engineering 11 (3) (2013) 201-225, ISSN 1543-1649.

[49] M. Bessa, R. Bostanabad, Z. Liu, A. Hu, D. W. Apley, C. Brinson, W. Chen, W. K. Liu, A framework for data-driven analysis of materials under uncertainty: Countering the curse of dimensionality, Computer Methods in Applied Mechanics and Engineering 320 (2017) 633 - 667, ISSN 0045-7825, doi:http://dx.doi.org/10.1016/j.cma.2017.03.037, URL http://www.sciencedirect.com/science/article/pii/S0045782516314803.

[50] L. Wu, C. Nghia Chung, Z. Major, L. Adam, L. Noels, A micromechanics-based inverse study for stochastic order reduction of elastic UD-fiber reinforced composites analyzes, International Journal for $\mathrm{Nu}-$ merical Methods in Engineering 115 (2018) 1430 - 1456, ISSN 0263-8223, doi:https://doi.org/10.1016/. 
[51] J. D. Eshelby, The Determination of the Elastic Field of an Ellipsoidal Inclusion, and Related Problems, Proceedings of the Royal Society of London. Series A, Mathematical and Physical Sciences 241 (1226) (1957) pp. 376-396, ISSN 00804630.

[52] T. Mori, K. Tanaka, Average stress in matrix and average elastic energy of materials with misfitting inclusions, Acta Metallurgica 21 (5) (1973) 571-574, cited By (since 1996) 1814.

[53] Y. Benveniste, A new approach to the application of Mori-Tanaka's theory in composite materials, Mechanics of Materials 6 (2) (1987) 147 - 157, ISSN 0167-6636, doi:DOI: 10.1016/0167-6636(87)90005-6.

[54] E. Kröner, Berechnung der elastischen Konstanten des Vielkristalls aus den Konstanten des Einkristalls, Zeitschrift für Physik A Hadrons and Nuclei 151 (1958) 504-518, ISSN 0939-7922, 10.1007/BF01337948.

[55] R. Hill, A self-consistent mechanics of composite materials, Journal of the Mechanics and Physics of Solids 13 (4) (1965) 213 - 222, ISSN 00225096, doi:DOI: 10.1016/0022-5096(65)90010-4.

[56] R. Hill, Continuum micro-mechanics of elastoplastic polycrystals, Journal of the Mechanics and Physics of Solids 13 (2) (1965) 89 - 101, ISSN 0022-5096, doi:DOI: 10.1016/0022-5096(65)90023-2.

[57] D. R. S. Talbot, J. R. Willis, Variational Principles for Inhomogeneous Non-linear Media, IMA Journal of Applied Mathematics 35 (1) (1985) 39-54, doi:10.1093/imamat/35.1.39.

[58] P. Ponte Castañeda, J. Willis, The effect of spatial distribution on the effective behavior of composite materials and cracked media, Journal of the Mechanics and Physics of Solids 43 (12) (1995) 1919 - 1951, ISSN 0022-5096, doi:https://doi.org/10.1016/0022-5096(95)00058-Q, URL http://www.sciencedirect.com/science/article/pii/002250969500058Q.

[59] I. Gitman, H. Askes, L. Sluys, Representative volume: Existence and size determination, Engineering Fracture Mechanics 74 (16) (2007) 2518 - 2534, ISSN 0013-7944, doi: http://dx.doi.org/10.1016/j.engfracmech.2006.12.021, URL http://www.sciencedirect.com/science/article/pii/S0013794406004772. 
[60] C. Soize, R. Ghanem, Data-driven probability concentration and sampling on manifold, Journal of Computational Physics $321 \quad$ (2016) $242 \quad$ - 258, ISSN 00219991, doi:https://doi.org/10.1016/j.jcp.2016.05.044, URL http://www.sciencedirect.com/science/article/pii/S0021999116301899.

[61] R. Peerlings, R. de Borst, W. Brekelmans, S. Ayyapureddi, Gradientenhanced damage for quasi-brittle materials, Int. J. Numer. Meth. Engng 39 (1996) 3391-3403.

[62] R. Peerlings, R. de Borst, W. Brekelmans, M. Geers, Gradient-enhanced damage modelling of concrete fracture, Mech. Cohesive-Frictional Mat. 3 (1998) 323-342.

[63] M. G. D. Geers, R. de Borst, W. A. M. Brekelmans, R. H. J. Peerlings, Strain-based transient-gradient damage model for failure analyses, Computer methods in applied mechanics and engineering 160 (1998) 133-153.

[64] R. Peerlings, M. Geers, R. de Borst, W. Brekelmans, A critical comparison of nonlocal and gradient-enhanced softening continua, Int. J. Solids Structures 38 (2001) 7723-7746.

[65] L. Wu, F. Sket, J. M. Molina-Aldareguia, A. Makradi, L. Adam, I. Doghri, L. Noels, A study of composite laminates failure using an anisotropic gradient-enhanced damage mean-field homogenization model, Composite Structures 126 (2015) 246 - 264, ISSN 0263-8223, doi:10.1016/j.compstruct.2015.02.070.

[66] J. Lemaitre, Coupled elasto-plasticity and damage constitutive equations, Computer Methods in Applied Mechanics and Engineering 51 (1-3) (1985) 31 - 49, ISSN 0045-7825, doi:DOI: 10.1016/0045$7825(85) 90026-\mathrm{X}$.

[67] I. Doghri, Numerical implementation and analysis of a class of metal plasticity models coupled with ductile damage, International Journal for Numerical Methods in Engineering 38 (20) (1995) 3403-3431, ISSN 1097-0207, doi:10.1002/nme.1620382004.

[68] D. Peric, E. A. de Souza Neto, R. A. Feijóo, M. Partovi, A. J. C. Molina, On micro-to-macro transitions for multi-scale analysis of nonlinear heterogeneous materials: unified variational basis and finite 
element implementation, International Journal for Numerical Methods in Engineering 87 (2010) 149 - 170, ISSN 1097-0207, URL http://dx.doi.org/10.1002/nme.3014.

[69] J. Schröder, M. Labusch, M.-A. Keip, Algorithmic two-scale transition for magneto-electro-mechanically coupled problems: FE2scheme: Localization and homogenization, Computer Methods in Applied Mechanics and Engineering 32 (2016) 253-280, ISSN 0045-7825, doi:http://dx.doi.org/10.1016/j.cma.2015.10.005, URL http://www.sciencedirect.com/science/article/pii/S0045782515003242.

[70] V.-D. Nguyen, L. Wu, L. Noels, Unified treatment of microscopic boundary conditions and efficient algorithms for estimating tangent operators of the homogenized behavior in the computational homogenization method, Computational Mechanics 59 (3) (2017) 483-505, ISSN 1432-0924, doi:10.1007/s00466-016-1358-z, URL https://doi.org/10.1007/s00466-016-1358-z.

[71] V.-D. Nguyen, L. Wu, L. Noels, Multiscale analyses of failure responses in fiber-reinforced composites, Mechanics of Materials - (-) (Submitted) - , ISSN -, doi:-.

[72] J. Segurado, J. Llorca, A numerical approximation to the elastic properties of sphere-reinforced composites, Journal of the Mechanics and Physics of Solids 50 (10) (2002) 2107 - 2121, ISSN 0022-5096.

[73] D. R. S. Talbot, J. R. Willis, Bounds and Self-Consistent Estimates for the Overall Properties of Nonlinear Composites, IMA Journal of Applied Mathematics 39 (3) (1987) 215-240, doi:10.1093/imamat/39.3.215.

[74] P. Ponte Castañeda, The effective mechanical properties of nonlinear isotropic composites, Journal of the Mechanics and Physics of Solids 39 (1) (1991) 45-71, ISSN 0022-5096, doi:DOI: 10.1016/00225096(91)90030-R.

[75] P. Ponte Castañeda, A New Variational Principle and its Application to Nonlinear Heterogeneous Systems, SIAM Journal on Applied Mathematics 52 (5) (1992) 1321-1341, ISSN 00361399.

[76] D. Talbot, J. Willis, Some simple explicit bounds for the overall behaviour of nonlinear composites, International Journal of Solids and 
Structures 29 (14-15) (1992) 1981 - 1987, ISSN 0020-7683, doi:DOI: 10.1016/0020-7683(92)90188-Y.

[77] I. Doghri, A. Ouaar, Homogenization of two-phase elasto-plastic composite materials and structures: Study of tangent operators, cyclic plasticity and numerical algorithms, International Journal of Solids and Structures 40 (7) (2003) 1681 - 1712, ISSN 0020-7683, doi:DOI: 10.1016/S0020-7683(03)00013-1.

[78] A. Molinari, F. El Houdaigui, L. Tóth, Validation of the tangent formulation for the solution of the non-linear Eshelby inclusion problem, International Journal of Plasticity 20 (2) (2004) 291 307, ISSN 0749-6419, doi:10.1016/S0749-6419(03)00038-X, URL http://www. sciencedirect.com/science/article/pii/S074964190300038X.

[79] L. Wu, L. Noels, L. Adam, I. Doghri, A combined incremental-secant mean-field homogenization scheme with per-phase residual strains for elasto-plastic composites, International Journal of Plasticity 51 (2013) 80-102, doi:10.1016/j.ijplas.2013.06.006.

[80] L. Wu, L. Noels, L. Adam, I. Doghri, An implicit-gradientenhanced incremental-secant mean-field homogenization scheme for elasto-plastic composites with damage, International Journal of Solids and Structures 50 (24) (2013) 3843 - 3860, ISSN 0020-7683, doi: 10.1016/j.ijsolstr.2013.07.022.

[81] L. Brassart, L. Stainier, I. Doghri, L. Delannay, A variational formulation for the incremental homogenization of elasto-plastic composites, Journal of the Mechanics and Physics of Solids 59 (12) (2011) 2455 2475, ISSN 0022-5096, doi:10.1016/j.jmps.2011.09.004.

[82] J. Chaboche, P. Kanouté, A. Roos, On the capabilities of mean-field approaches for the description of plasticity in metal matrix composites, International Journal of Plasticity 21 (7) (2005) 1409 - 1434, ISSN 07496419, doi:DOI: 10.1016/j.ijplas.2004.07.001.

[83] L. Wu, L. Adam, I. Doghri, L. Noels, An incrementalsecant mean-field homogenization method with second statistical moments for elasto-visco-plastic composite materials, 
Mechanics of Materials $114 \quad(2017) \quad 180-200$, ISSN 01676636, doi:https://doi.org/10.1016/j.mechmat.2017.08.006, URL http://www. sciencedirect.com/science/article/pii/S0167663617300698.

[84] O. Pierard, I. Doghri, Study of Various Estimates of the Macroscopic Tangent Operator in the Incremental Homogenization of Elastoplastic Composites, International Journal for Multiscale Computational Engineering 4 (4) (2006) 521-543, ISSN 1543-1649.

[85] I. Doghri, L. Brassart, L. Adam, J. S. Gérard, A second-moment incremental formulation for the mean-field homogenization of elasto-plastic composites, International Journal of Plasticity 27 (3) (2011) 352 - 371, ISSN 0749-6419, doi:DOI: 10.1016/j.ijplas.2010.06.004.

[86] T. H. Hoang, M. Guerich, J. Yvonnet, Determining the Size of RVE for Nonlinear Random Composites in an Incremental Computational Homogenization Framework, Journal of Engineering Mechanics 142 (5) (2016) 04016018, doi:10.1061/(ASCE)EM.1943-7889.0001057.

[87] S. Yang, S. Yu, J. Ryu, J.-M. Cho, W. Kyoung, D.-S. Han, M. Cho, Nonlinear multiscale modeling approach to characterize elastoplastic behavior of $\mathrm{CNT} /$ polymer nanocomposites considering the interphase and interfacial imperfection, International Journal of Plasticity 41 (2013) 124 - 146, ISSN 0749-6419, doi:https://doi.org/10.1016/j.ijplas.2012.09.010, URL http://www.sciencedirect.com/science/article/pii/S0749641912001404.

[88] J. Lemaitre, R. Desmorat, Engineering damage mechanics: ductile, creep, fatigue and brittle failures, Springer-Verlag, Berlin, ISBN 3540215034, 2005.

[89] M. Mosby, K. Matouš, On mechanics and material length scales of failure in heterogeneous interfaces using a finite strain high performance solver, Modelling and Simulation in Materials Science and Engineering 23 (8) (2015) 085014, URL http://stacks. iop.org/0965-0393/23/i=8/a=085014.

[90] V. P. Nguyen, O. Lloberas-Valls, M. Stroeven, L. J. Sluys, On the existence of representative volumes for softening quasi-brittle materials A failure zone averaging scheme, Computer Methods in 
Applied Mechanics and Engineering 199 (4548) (2010) 3028 - 3038, ISSN 0045-7825, doi:http://dx.doi.org/10.1016/j.cma.2010.06.018, URL http://www.sciencedirect.com/science/article/pii/S0045782510001854.

[91] C. V. Verhoosel, J. J. C. Remmers, M. A. Gutiérrez, R. de Borst, Computational homogenization for adhesive and cohesive failure in quasibrittle solids, International Journal for Numerical Methods in Engineering 83 (8-9) (2010) 1155-1179, ISSN 1097-0207, doi:10.1002/nme.2854, URL http://dx.doi.org/10.1002/nme.2854.

[92] L. Wu, D. Tjahjanto, G. Becker, A. Makradi, A. Jérusalem, L. Noels, A micromeso-model of intra-laminar fracture in fiber-reinforced composites based on a discontinuous Galerkin/cohesive zone method, Engineering Fracture Mechanics 104 (2013) 162 - 183, ISSN 00137944, doi:https://doi.org/10.1016/j.engfracmech.2013.03.018, URL http://www.sciencedirect.com/science/article/pii/S0013794413001252.

[93] N. Nguyen, J. Yvonnet, J. Réthoré, A. B. Tran, Identification of fracture models based on phase field for crack propagation in heterogeneous lattices in a context of non-separated scales, Computational Mechanics ISSN 1432-0924, doi:10.1007/s00466-018-1636-z, URL https://doi.org/10.1007/s00466-018-1636-z.

[94] L. Wu, G. Becker, L. Noels, Elastic damage to crack transition in a coupled non-local implicit discontinuous Galerkin/extrinsic cohesive law framework, Computer Methods in Applied Mechanics and Engineering 279 (2014) 379 - 409, ISSN 00457825, doi:http://dx.doi.org/10.1016/j.cma.2014.06.031, URL http://www.sciencedirect.com/science/article/pii/S0045782514002175.

[95] J. Leclerc, L. Wu, V. D. Nguyen, L. Noels, A damage to crack transition model accounting for stress triaxiality formulated in a hybrid nonlocal implicit discontinuous Galerkincohesive band model framework, International Journal for Numerical Methods in Engineering 113 (3) (2018) 374-410, doi:10.1002/nme.5618, URL https://onlinelibrary.wiley.com/doi/abs/10.1002/nme.5618.

[96] I. Doghri, Mechanics of Deformable Solids- Linear, Nonlinear, Analytical and Computational Aspects, Springer-Verlag, Berlin, ISBN 3540669604, 2000 . 
[97] J. C. Simo, R. L. Taylor, Consistent tangent operators for rateindependent elastoplasticity, Computer Methods in Applied Mechanics and Engineering 48 (1) (1985) 101 - 118, ISSN 0045-7825, doi:DOI: 10.1016/0045-7825(85)90070-2. 